\title{
Multi-Parameter Fiber Optic Sensors for Structural Health Monitoring
}

\author{
by \\ Alexi Ivanov, B. Eng. \\ A thesis submitted to \\ The Faculty of Graduate Studies and Research \\ In partial fulfillment of the requirements for the degree of \\ Master of Applied Science
}

Ottawa-Carleton Institute for Electrical and Computer Engineering

Department of Electronics

Faculty of Engineering

Carleton University

Ottawa, Ontario, Canada

April 2008

(C) Alexi Ivanov, 2008 


$\begin{array}{ll}\begin{array}{l}\text { Library and } \\ \text { Archives Canada }\end{array} & \begin{array}{l}\text { Bibliothèque et } \\ \text { Archives Canada }\end{array} \\ \begin{array}{l}\text { Published Heritage } \\ \text { Branch }\end{array} & \begin{array}{l}\text { Direction du } \\ \text { Patrimoine de l'édition }\end{array} \\ \begin{array}{l}\text { 395 Wellington Street } \\ \text { Ottawa ON K1A 0N4 } \\ \text { Canada }\end{array} & \begin{array}{l}\text { 395, rue Wellington } \\ \text { Ottawa ON K1A 0N4 } \\ \text { Canada }\end{array}\end{array}$

Your file Votre référence ISBN: 978-0-494-40640-3

Ourfile Notre référence

ISBN: 978-0-494-40640-3

NOTICE:

The author has granted a nonexclusive license allowing Library and Archives Canada to reproduce, publish, archive, preserve, conserve, communicate to the public by telecommunication or on the Internet, loan, distribute and sell theses worldwide, for commercial or noncommercial purposes, in microform, paper, electronic and/or any other formats.

The author retains copyright ownership and moral rights in this thesis. Neither the thesis nor substantial extracts from it may be printed or otherwise reproduced without the author's permission.
AVIS:

L'auteur a accordé une licence non exclusive permettant à la Bibliothèque et Archives Canada de reproduire, publier, archiver, sauvegarder, conserver, transmettre au public par télécommunication ou par l'Internet, prêter, distribuer et vendre des thèses partout dans le monde, à des fins commerciales ou autres, sur support microforme, papier, électronique et/ou autres formats.

L'auteur conserve la propriété du droit d'auteur et des droits moraux qui protège cette thèse. $\mathrm{Ni}$ la thèse ni des extraits substantiels de celle-ci ne doivent être imprimés ou autrement reproduits sans son autorisation.
In compliance with the Canadian Privacy Act some supporting forms may have been removed from this thesis.

While these forms may be included in the document page count, their removal does not represent any loss of content from the thesis.
Conformément à la loi canadienne sur la protection de la vie privée, quelques formulaires secondaires ont été enlevés de cette thèse.

Bien que ces formulaires aient inclus dans la pagination, il n'y aura aucun contenu manquant.

\section{Canada}




\section{Abstract}

Structural health monitoring (SHM) is becoming an increasingly preferred approach for preventative maintenance in many industries. It involves distributed monitoring of structural shape, stress and temperature. Conventionally electrical sensors have been used to perform those kind of measurements however on the large scale copper wires associated with many electrical sensors limit the integration and quite often can not withstand harsh operational requirements. Fiber optic sensors have proven to offer certain advantages however more work remains to make them suitable for multi parameter, distributed sensing.

In this Thesis the temperature and strain sensitivities of regular fiber Bragg gratings (FBG) with acrylic and polyamide coatings, center wavelengths within the C-band and dual and single configurations are determined. Different coatings were found to impact the sensitivities the most while different wavelengths have essentially the same sensitivities, which makes dual grating based temperature strain discrimination impossible. Novel tilted FBGs have also been explored. They allow coupling into the backward propagating cladding modes which experience both power and wavelength shifts due to applied strain, temperature and bending. This offers unique multi parameter discrimination and simplifies sensor interrogation. As a proof of concept temperature independent bend and vibration sensors are demonstrated. 


\section{Acknowledgements}

I would like to thank my supervisor Dr. Jacques Albert for offering me this challenging and rewarding opportunity. His professionalism and motivation made it very easy for me to go through the process of graduate school. I would also like to thank Dr. Nezih Mrad for introducing me to the FBG structural health monitoring project from which I learned so much.

My gratitude goes out to all those great people who helped along the way. Especially I would like to thank Andrew Furlong for giving me a crash course on material science, helping me fabricate test templates in the machine shop and teaching me how to bond resistive strain gauges. Many thanks are owed to Albane Laronche for her magical ability to make equipment work and endless supply of fiber Bragg gratings. I am also very grateful to the staff in the Carleton university materials testing lab for letting me use their equipment and spending their time with me.

I am also very thankful to the members of my research group Alexander Andreyuk, Lingyun Xiong, Yanina Shevchenko, Ksenia Yadav, Chengkun Chen, Tuan Guo and Rodica Rogojan for creating a great working environment.

Finally I would like to thank my family for being there every step of the way providing their support when I needed it the most. 


\section{Table of Contents}

Abstract........................................................................................................................................ii

Acknowledgements ........................................................................................................................... iv

Table of Contents .................................................................................................................................... v

List of Tables ........................................................................................................................................ ix

List of Figures................................................................................................................................. $\mathrm{x}$

Chapter 1: Introduction ........................................................................................................ 1

Chapter 2: Structural Health Monitoring: Requirements and Solutions. ..................... 4

2.1 Structural Health Monitoring Requirements......................................................... 4

2.1.1 Static Strain Monitoring....................................................................... 5

2.1.2 Dynamic Strain Monitoring ....................................................................... 6

Natural Frequency Based Methods ..........................................................................................

Frequency Mode Shape Based Methods .......................................................................................

Vibration Detection in Rotary Blades ...................................................................................

High Frequency Vibration...................................................................................................

2.1.3 Curvature and Shape Monitoring................................................................. 8

2.2 Structural health monitoring solutions.................................................................. 9

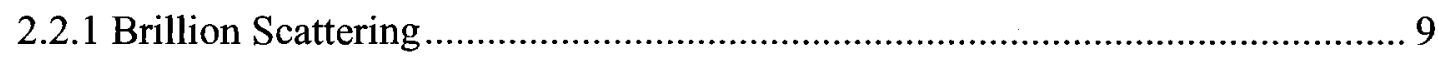

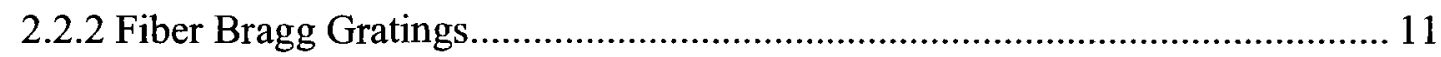

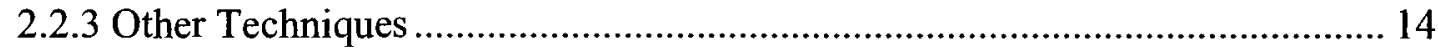

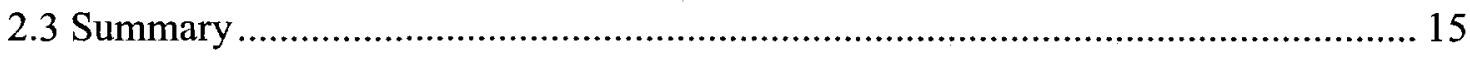


Chapter 3: Fiber Bragg Grating Theory ….................................................................................. 17

3.1 Introduction to Fiber Bragg Gratings.................................................................. 17

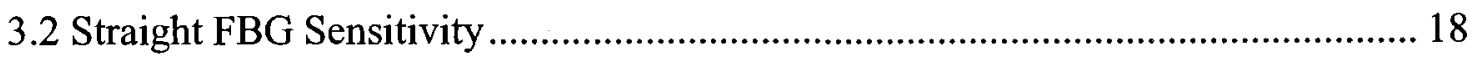

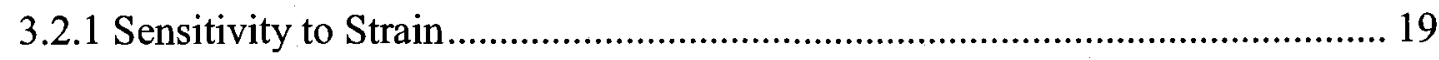

3.2.2 Sensitivity to Temperature........................................................................... 22

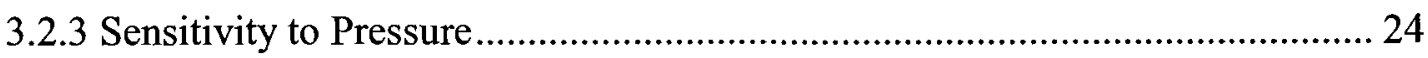

3.2.4 Dual Grating Temperature-Strain Discrimination ........................................... 25

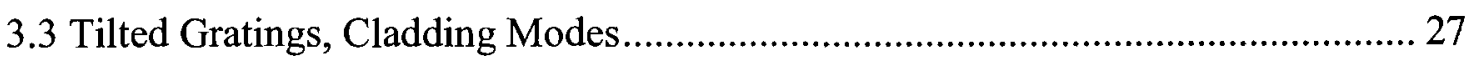

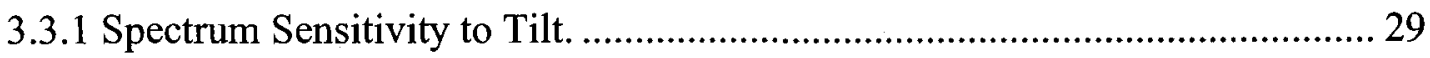

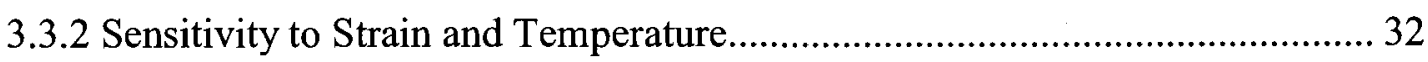

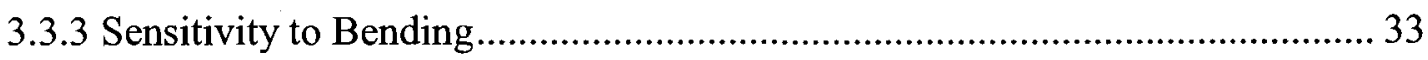

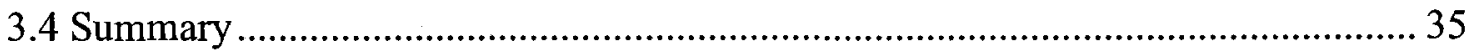

Chapter 4: Free and Bonded Sensitivity and Temperature/Strain Discrimination

Using Dual FBGs...................................................................................................................... 37

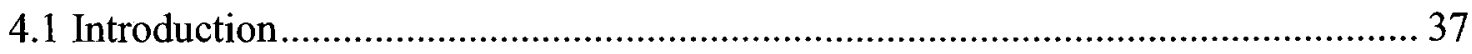

4.2 Free Temperature and Strain Sensitivity Characterization of Free FBGs. .............. 37

4.2.1 Strain Characterization Procedure and Results............................................... 38

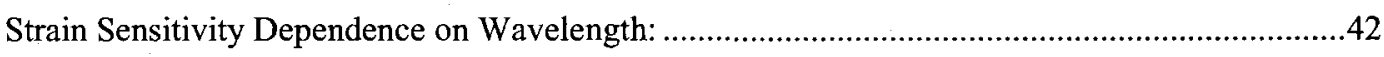

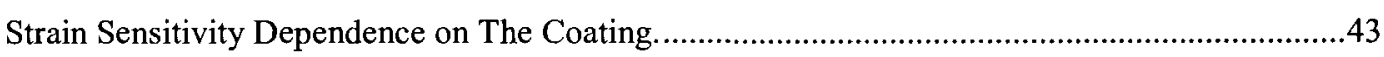

Strain Sensitivity Dependence on The Configuration..................................................................44

4.2.2 Temperature Characterization Procedure and Results...................................... 44

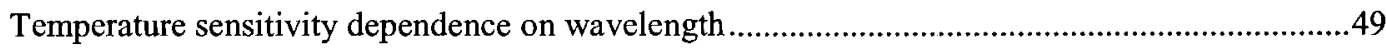

Temperature Sensitivity Dependence on Coating .....................................................................49

Temperture Sensitivity Dependence on Configuration ..................................................................50

Wavelength Response For an Extended Temperature Range ..................................................50

4.2.3 Combined Temperature and Strain Characterization......................................... 51

4.2.4 Dual Grating Error Analysis ..........................................................................5

4.3 Bonded Temperature and Strain Sensitivity Characterization................................. 56

4.3.1 Bonding Procedure........................................................................................ 56 


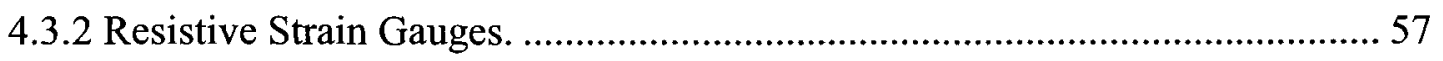

4.3.3 Bonded Strain Characterization Procedure and Results .................................... 60

Strain Sensitivity Dependence on Wavelength: .............................................................................65

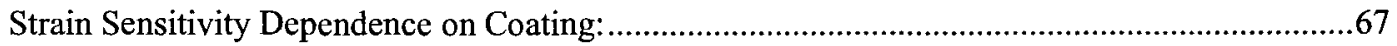

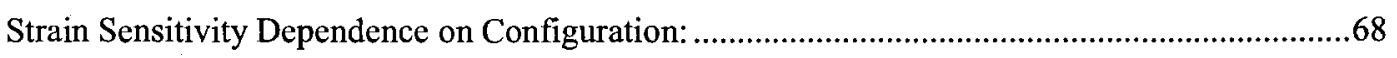

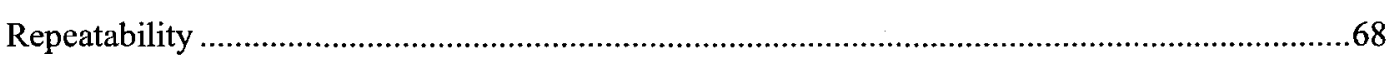

4.3.4 Bonded Temperature Characterization Procedure and Results ........................ 69

Temperature Sensitivity Dependence on Wavelength ................................................................72

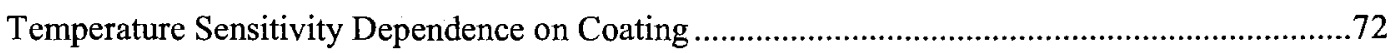

Temperature Sensitivity Dependence on Configuration ..................................................................72

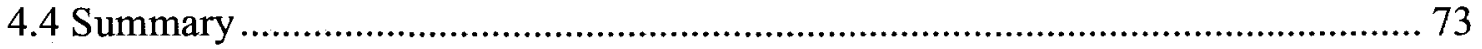

Chapter 5: Tilted Gratings, Vibration and Bend sensing .............................................. 74

5.1 Tilted Grating Fabrication............................................................................... 74

5.2 Cladding Mode Sensitivity to Bending, in Transmission...................................... 75

5.2.1 Large Curvature Bending Experimental Set-up.............................................. 76

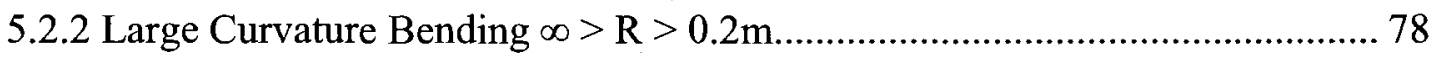

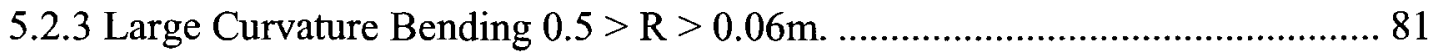

5.2.4 Small Curvature Bending 0.05m $>\mathrm{R}>0.02 \mathrm{~m}$............................................. 85

5.2.5 Proof of Concept, Temperature Independent Strain Gauge Demonstration.... 91

5.2.6 Temperature-Strain Discrimination .......................................................... 93

5.3 Ghost Mode Sensitivity to Bending in Reflection................................................. 95

5.3.1 Ghost Mode Recoupling ............................................................................. 95

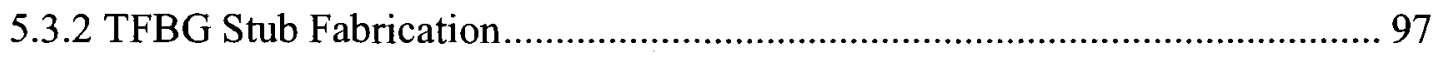

5.3.3 Temperature and Bending Results .............................................................. 98

5.3.4 Proof of Concept Vibration Sensor Demonstration........................................ 101

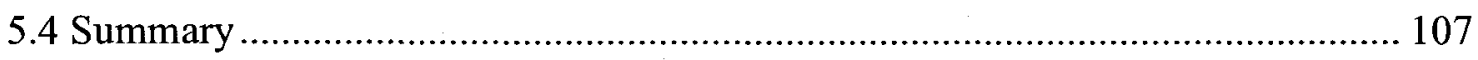

Chapter 6: Conclusions and future work ................................................................... 108

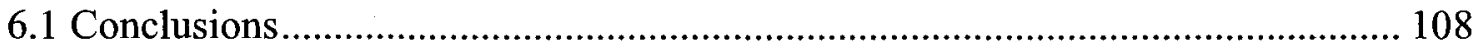

6.2 Recommendations for Future Work.................................................................. 109 
Chapter 7: Publications and Patents........................................................................ 111

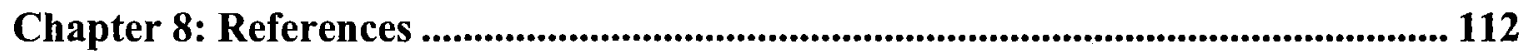




\section{List of Tables}

Table 2.1: Sensor classification by the frequency range ................................................ 4

Table 3.1: Summary of Thermo-optic coefficients of silica ............................................ 23

Table 3.2: Summary of dual grating pair condition numbers........................................ 26

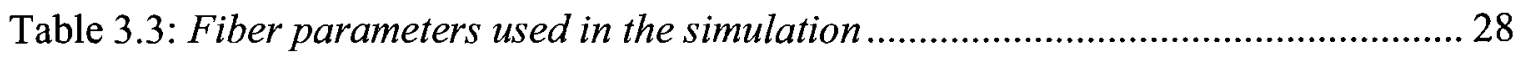

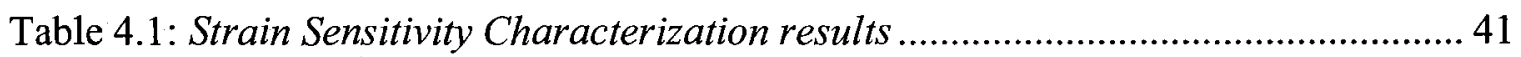

Table 4.2: Previously reported strain sensitivity ..................................................... 42

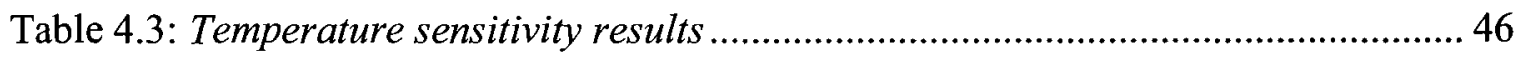

Table 4.4: Previously reported temperature sensitivities ............................................ 47

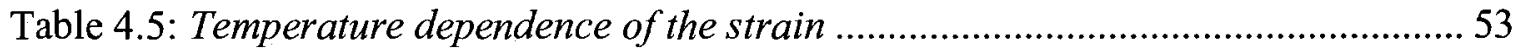

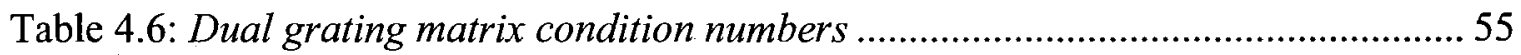

Table 4.7: Applied and calculated temperature and strain ....................................... 56

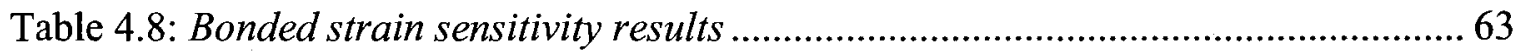

Table 4.9: Retest results on FOS2 with a spectrum analyzer ....................................... 66

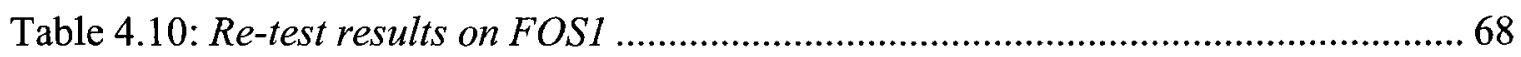

Table 4.11: Bonded Temperature sensitivity results ..................................................... 70 


\section{List of Figures}

Figure 3.1: Tilted Fiber Bragg grating plane orientation .......................................... 18

Figure 3.2: Expected Strain sensitivity of a FBG for a range of wavelengths ................. 20

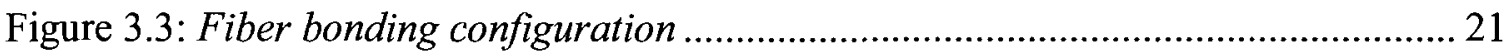

Figure 3.4: Wavelength dependence of the temperature sensitivity ............................. 24

Figure 3.5: TFBG transmission and reflection spectrum for a $3^{\circ}$ grating ....................... 28

Figure 3.6: Optigrating transmission spectrum for $a 3^{\circ}$ TFBG ................................. 29

Figure 3.7:Grating spectrum evolution for various tilt angles .................................... 30

Figure 3.8: Break down of the modes which make up the Ghost ................................... 31

Figure 3.9: Peak loss for a selection of modes and tilt angles...................................... 32

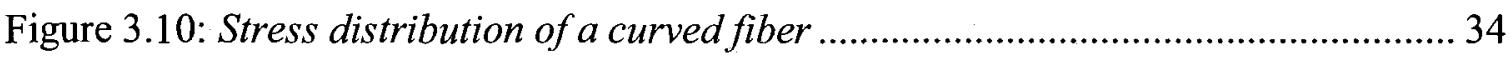

Figure 3.11: Mode intensity profiles ...................................................................... 35

Figure 4.1: Strain characterization experimental set-up ........................................... 38

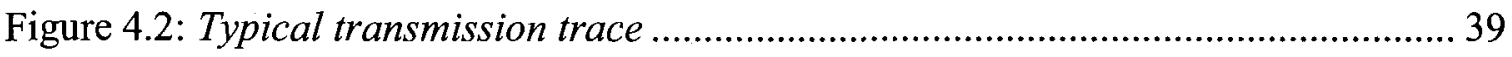

Figure 4.3: Typical Extension vs. Wavelength Plot ............................................ 40

Figure 4.4: Strain sensitivity vs. wavelength for single gratings ................................. 42

Figure 4.5: Strain sensitivity vs. wavelength for dual gratings..................................... 43

Figure 4.6: Uninsulated Peltier heater ................................................................ 45

Figure 4.7: Typical temperature response, polyamide coating................................... 45

Figure 4.8: Temperature sensitivity for single gratings ......................................... 48

Figure 4.9: Temperature sensitivity for serial and collocated gratings...................... 49

Figure 4.10: Temperature sensitivity of the Acrylic and Polyamide coated .................... 51

Figure 4.11: Combined temperature and strain experimental set-up ............................ 52

Figure 4.12: Temperature dependence of the strain sensitivity ..................................... 54

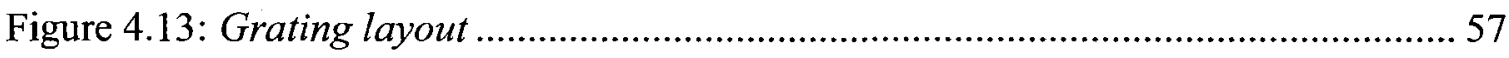




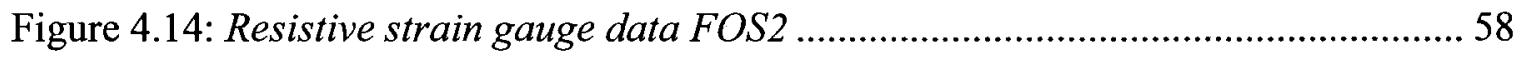

Figure 4.15: Resistive strain gauge FOS2 after the front gauge has been replaced ........ 59

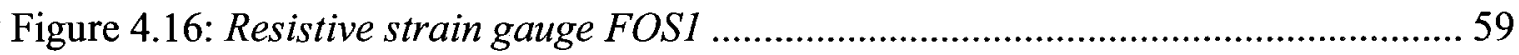

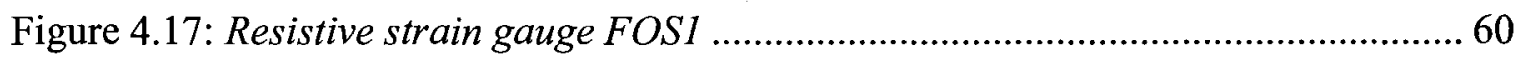

Figure 4.18: Repeatability results for polyamide coated dual FBG on FOS2 _.............. 61

Figure 4.19: Repeatability results for acrylate coated single FBG on FOS1 .................. 62

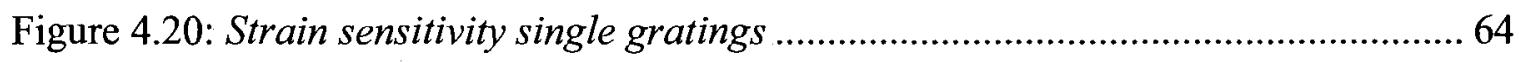

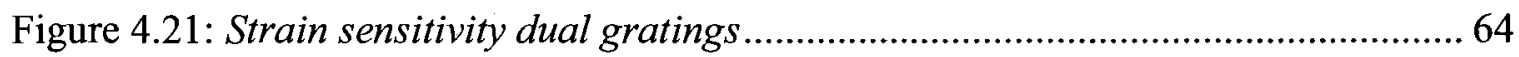

Figure 4.22: Reflection spectrum for two different loads, measured with si720 ............ 66

Figure 4.23: Superimposed reflection spectrum for two different loads, measured with

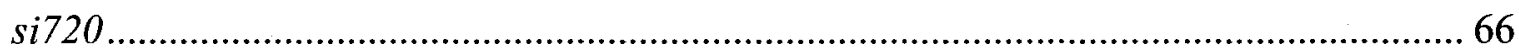

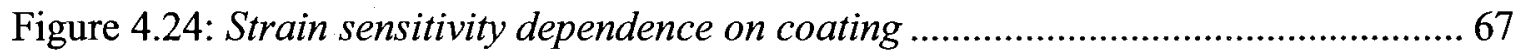

Figure 4.25: Reflection spectrum of a $1540 \mathrm{~nm}$ bare grating ..................................... 69

Figure 4.26: Bonded temperature sensitivity for single gratings................................. 71

Figure 4.27: Bonded temperature sensitivity for dual gratings ................................... 71

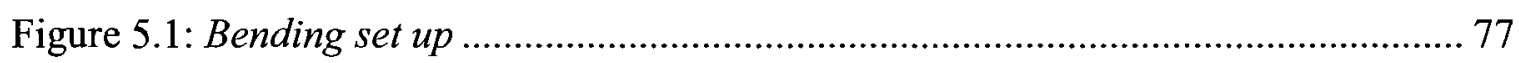

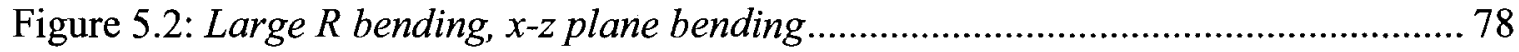

Figure 5.3: Large R bending, $y$-z plane bending....................................................... 79

Figure 5.4:Cladding mode coupling sensitivity versus bending for two different

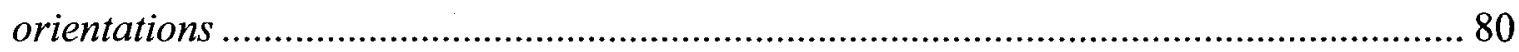

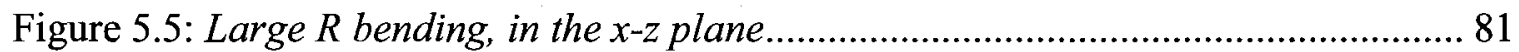

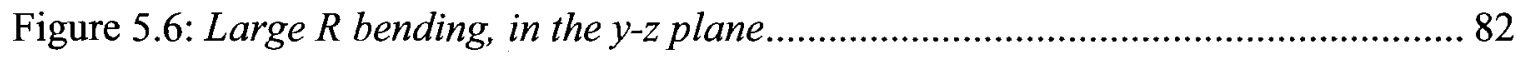

Figure 5.7: Repeatability comparison of the two spectrums .......................................... 83

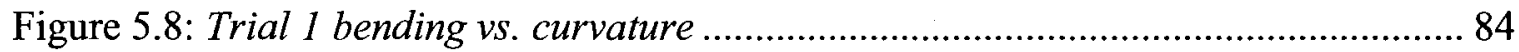

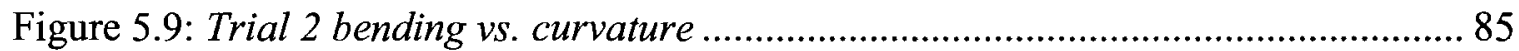

Figure 5.10:Transmission spectrum for the curvature ranging from 45.5 to $43 \mathrm{~mm}$........ 86

Figure 5.11:Transmission spectrum for the curvature ranging 28.5 to $25 \mathrm{~mm}$................. 87

Figure 5.12:Temperature sensitivity of a $T F B G$ coiled with a $27 \mathrm{~mm}$ radius................... 88

Figure 5.13:Ghost mode bending sensitivity $x$-z plane .............................................. 89 
Figure 5.14:Ghost mode bending sensitivity $y$-z plane …………..................................... 89

Figure 5.15: Ghost mode power coupling vs. the bending radius .................................... 90

Figure 5.16: Ghost mode coupling vs. the bending radius for two different gratings ...... 90

Figure 5.17:TFBG mounting set-up ............................................................................. 91

Figure 5.18: Transmission spectrum evolution of a $3^{\circ}$ TFBG ….................................... 92

Figure 5.19: Ghost mode power differential vs. strain ..................................................... 93

Figure 5.20: Ghost mode power differential vs. temperature ............................................. 94

Figure 5.21: Schematic representation of cladding mode recoupling .............................. 96

Figure 5.22: Ghost mode recoupling through slight misalignment of the junction ........... 97

Figure 5.23: Deflection mechanism ...................................................................... 98

Figure 5.24: Reflection spectrum of a TFBG stub at three different temperatures .......... 99

Figure 5.25: Reflection spectrum of a TFBG at room temperature and three different

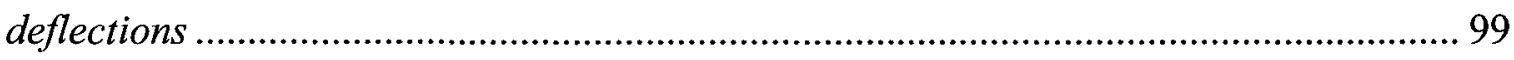

Figure 5.26: Power versus deflection at three different temperatures............................ 100

Figure 5.27: $3^{\circ}$ tilted grating transmission spectrum ................................................... 101

Figure 5.28: Reflection spectrum with the bad splice ................................................... 102

Figure 5.29: Recoated TFBG reflection spectrum ………........................................... 103

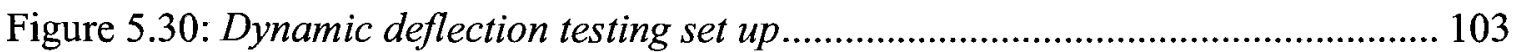

Figure 5.31: Vibration responce Bragg and Ghost modes .............................................. 104

Figure 5.32: Grating reflection spectrum illuminated by the multi line laser source .... 106

Figure 5.33: TFBG electrical response to vibration ........................................................ 106 


\section{Chapter 1:Introduction}

Active Structural Health Monitoring (SHM) is becoming the mainstream approach in prolonging the life of assets and increasing the operational safety and efficiency in many industries. To date structural monitoring is used in civil engineering, aerospace and oil exploration industries. It is also starting to appear in the automotive industry. These industries have very diverse sensing requirements due to the differences in operational conditions and the level of sensor integration that needs to be achieved. In the early works, the common approach was to use electrical sensing methods due to the maturity of the technology and low cost of the instrumentation, however for large scale SHM it became quickly apparent that electrical methods have a number of significant limitations. Even though conventional electrical sensors can be made very small each one of them has to be wired separately, this poses a significant problem for embedding quasi-distributed systems due to a large number of wires that need to be accommodated and the increasing total weight. SHM systems are also often required to survive very harsh environments such as deep underneath the ground in the oil well or submerged in corrosive sea water or even extremely cold temperatures of superconducting magnets and space exploration. Since electrical sensors are typically metal resistive strain gauges they do not survive these types of environments. Finally, electrical gauges don't work in the presence of strong electromagnetic interference such as the one caused by high power lines. Because of all of the above most recent efforts are concentrated on the fiber optic sensors. 
Using various multiplexing techniques such as time or frequency domain many sensors can be placed on a single fiber optic line minimizing the size, weight and the intrusion of the system. The fiber composition is silica glass and therefore it can withstand very harsh environments, which most metals can not. Finally fibers carry light as opposed to electrical current making fiber optic sensors intrinsically immune to electromagnetic interference. Fiber Bragg Gratings (FBGs) have already emerged as the leader in fiber optic SHM solutions. However one of the major drawbacks of FBGs is cross sensitivity to strain and temperature and the difficulty in measuring vibrations and bends.

The objective of this Thesis is to develop simple Fiber Bragg Grating (FBG) based sensor designs for SHM which would capitalize on the advantages of optical sensors and at the same time allow to discriminate between multiple parameters. The organization will be as follows. The next chapter will provide a literature review of SHM approaches, sensor requirements and solutions that have been developed up until now. The third chapter will contain the theory behind FBG sensitivity to temperature, strain and bending. The fourth chapter will present the experimentally determined temperature and strain sensitivities for free and bonded FBG as well as the comparison of the sensitivity of FBGs with different wavelengths, coatings and configurations. Finally the limitations of the closely spaced dual grating temperature/strain discrimination method will be discussed. The fifth chapter will explore novel tilted FBGs. The tilt angle causes significant coupling to the cladding modes which simplify temperature discrimination and allows the detection of extra parameters such as bending. As a proof of concept a TFBG based temperature independent strain gauge and intrinsic vibration sensor 
interrogated by an inexpensive photodiode set-up will be demonstrated. Final conclusions and recommendations for future work will be presented in chapter 6 . 


\section{Chapter 2: Structural Health Monitoring: Requirements and Solutions.}

SHM is a very diverse field which requires diverse solutions but at the same time there are some traits which are common for many applications. In this chapter the SHM applications will be classified according to the common requirements, then the sensors, which have been developed up to now, will be covered, discussing their advantages and limitations.

\subsection{Structural Health Monitoring Requirements.}

In general sensor applications can be classified as shown in Table 2.1 into four main groups by their measurement frequency, Hongo et al. (2005).

\begin{tabular}{|c|c|c|c|c|}
\hline $\begin{array}{l}\text { Frequency } \\
\text { range }\end{array}$ & $1 \mathrm{~Hz}$ & $100 \mathrm{~Hz}$ & $1 \mathrm{kHz}$ & $I M H z$ \\
\hline $\begin{array}{l}\text { Application } \\
\text { field }\end{array}$ & $\begin{array}{l}\text {-Static strain, } \\
\text { temperature, etc. } \\
\text { monitoring for } \\
\text { large } \\
\text { structures, } \\
\text { oilfield } \\
\text { explorations, } \\
\text { aerospace and } \\
\text { composites. } \\
\text { (E.g. buildings, } \\
\text { tunnels, dams) }\end{array}$ & $\begin{array}{l}\text {-SHM via } \\
\text { vibration } \\
\text { monitoring of } \\
\text { civil and } \\
\text { aerospace } \\
\text { structures. }\end{array}$ & $\begin{array}{l}\text {-Accelerometers } \\
\text {-Seismographs } \\
\text {-Vibration } \\
\text { monitoring of } \\
\text { power cables } \\
\text { trolley cables, } \\
\text { pipelines, etc. }\end{array}$ & $\begin{array}{l}\text {-Impact damage } \\
\text { detection } \\
\text {-Hydrophones } \\
\text {-Acoustic } \\
\text { emission } \\
\text { spectroscopy } \\
\text { for non- } \\
\text { destructive } \\
\text { evaluation }\end{array}$ \\
\hline
\end{tabular}

Table 2.1: Sensor classification by the frequency range 
The following sub sections will describe each subgroup in detail.

\subsubsection{Static Strain Monitoring}

The focus of many works in structural health monitoring is localized parameter monitoring such as strain, temperature or pressure. One application where such monitoring is useful is fabrication of composite materials. Process induced strains during cure can lead to significant problems, such as warpage or spring-in, which present difficulties in the assembly of composite structures. Therefore a precise knowledge of curing conditions such as pressure temperature and strain inside a laminate is required, Guo (2007). The sensor for such application must be able to survive the curing temperatures and be capable of discriminating between multiple parameters.

Local stain monitoring can also be used for crack detection in large scale civil engineering structures. Alam \& Swamidas, (2002) have determined through simulations that large changes in normalized local strains develop "near" and "far-away" from the high stress point in steel jacket offshore platforms. Moreover, characteristic displacement and strain changes occur all over the damaged structure that can be utilized, in conjunction with a trained neural network procedure, to identify the crack size and location. The sensor again must withstand the harsh environments and operate over a large temperature range.

Another application of static SHM is oil exploration industry. Skinner \& Maida, (2004) provide a good overview of borehole sensor specifications. In this industry, distributed temperature and pressure sensors are typically required. Again the main challenge is cross sensitivity to multiple parameters, simplicity and low cost of the sensor 
as well as high reliability down in the bore hole. Another very important aspect is safety: a passive, non-electrical sensor is preferred.

Monitoring of superconducting magnets and liquid nitrogen storage tanks at cryogenic temperatures has also been extremely difficult due to complex temperature compensation schemes and strong electromagnetic interference.

\subsubsection{Dynamic Strain Monitoring}

The other very widespread approach in structural heath monitoring is vibration analysis. First studies on the use of vibration to monitor structural integrity have been done by Lifshitz \& Rotem (1969). A good summary of vibration based SHM is done by Doebling et al. (1996) and then updated by Carden \& Fanning (2004). The basic idea behind vibration analysis is that modal parameters (frequency, modal shape, modal damping) are functions of the physical parameters and therefore changes in physical parameters, which usually lead to structural failures, will cause changes in modal parameters, which are easy to detect using specialized sensor networks.

From the sensor output and configuration point of view all the methods can be classified into two groups: natural frequency and frequency mode shape based methods. The damage is also classified as linear (structural properties remain linear after damage) or non-linear (cracks, loose connections), Doebling et al. (1996).

\section{Natural Frequency Based Methods}

In this method the damage is detected via the change of the natural frequencies of vibration of a structure. The advantage is that only a single sensor is required to monitor 
the whole structure. However, Chen et al. (1995), have demonstrated that by using natural frequencies alone it is quite difficult to determine structural failure. For example the first four frequencies of a steel channel exhibited no shifts greater than $5 \%$, due to a single notch severe enough to cause the channel to fail at its design load. Given that it is acknowledged that frequency variation due to incidental/ambient vibration and environmental effects can be as high as $5-10 \%$, they argued that lower frequency shifts would not necessarily be useful damage indicators. However, successful lab results and small scale tests have been carried out and natural frequency shift due to damage has been proven detectable with fiber optic sensor systems Calvert \& Mooney, (2004).

Another significant drawback of the natural frequency based methods is the difficulty of localising the damage, therefore according to Carden \& Fanning, (2004) the suitability of frequency shifts alone for identifying damage in full-scale structures, based on the literature to date, does not seem promising.

\section{Frequency Mode Shape Based Methods}

Measurement of the mode shapes of a structure requires either a single excitation point and many sensors or a roving exciter with one or more fixed sensors. Calvert \& Mooney (2004) have reported that damage in a concrete structure had resulted in curvature changes of the mode shapes and modal macro-strain vectors detectable by fiber optic sensors. The biggest issue here is correlating the damage with the change in mode shapes and difficulties related to integration of a large sensor network. Environmental effects and structural alterations are also all known to affect the mode shapes and therefore must be kept in mind when designing the sensor head. Carden \& Fanning, 
(2004) point out that this area has been neglected by the majority of the authors and requires the most development in order for the vibration based methods to advance.

\section{Vibration Detection in Rotary Blades}

Recently there has also been a significant amount of interest in active vibration compensation of rotary structures such as helicopter blades Yong et al. (2004). Sensor array network can be embedded in the structure and provide the vibration information to a feedback loop. Actuators could then be used to alter the mechanical properties of the structure to dampen the unwanted vibrations. The key requirements are low weight, survivability in harsh environments and distributed sensing Garg et al. (2002) which all fit very well with fiber optic sensor concepts.

\section{High Frequency Vibration}

Capoluongo et al. (2005) have shown that modal shape analysis can be successfully used to detect damages at frequencies up to $2 \mathrm{KHz}$. Sampling frequencies of up to $20 \mathrm{kHz}$ have been achieved for impact detection, modal analysis and dynamic load measurements, Cheng (2005). Finally ultrasonic Lamb waves with the frequencies ranging from $100 \mathrm{kHz}$ up to $\mathrm{MHz}$ have been detected by Betz et al. (2003) for the purpose of SHM. Finally a temperature independent ultrasonic sensor with an upper frequency response of several MHz has been demonstrated by Fisher et al. (1998).

\subsubsection{Curvature and Shape Monitoring}

Curvature contains information on structural integrity. In many physical structures, particularly those in which size and weight are critical, the monitoring of strain and 
bending to make real-time shape measurements offers several performance advantages, beyond that of simply avoiding failure. In acoustic and electromagnetic antennae, monitoring deformation allows shape changes to be compensated in subsequent signal processing. Deformation measurements can also be used as inputs to active damping and shape control systems to control shape directly, or to locate the position of the end of a flexible member, Fender et al. (2006)

\subsection{Structural health monitoring solutions}

As can be seen from the previous section one of the factors which are limiting the extent of the SHM is the lack of a universal sensor that would be capable to perform distributed, multi-parameter monitoring under very harsh conditions while keeping the overall system compact and inexpensive. Harsh environments, electromagnetic interference and small system intrusion often rule out the use of conventional electrical sensors. On the other hand, fiber-optic sensors are starting to attract more and more attention since they can be easily multiplexed and integrated in many structures. This section will provide an overview of most successful fiber-optic technologies which have been used for SHM in the past. The advantages and disadvantages of each technique will be discussed.

\subsubsection{Brillion Scattering}

One type of successful fiber optic sensors is based on Brillion scattering. In distributed Brillion sensor (DBS) systems the actual sensing medium is a standard, single mode fiber. Two laser beams are launched into both ends of the fiber in a counter propagating 
fashion, When they interact there is energy transfer from the high frequency pump beam to the low frequency probe beam. The amount of loss is dependent on the frequency difference of the two beams. Maximum loss occurs at the Brillouin frequency, $v_{B}$, which is the acoustic phonon frequency. The loss is then plotted versus the frequency difference at different locations of the fiber. The spatial resolution is typically $15 \mathrm{~cm}$ and is determined by the pulse width, Ravet et al. (2007). Equations 2.1 and 2.2 show the dependence of $v_{B}$ and $P_{B}$ on temperature and strain applied to the fiber, Bao (2006).

$$
\begin{aligned}
& v_{B}=v_{B_{o}}+\frac{\partial v}{\partial \varepsilon} \varepsilon(\mu \varepsilon)+\frac{\partial v}{\partial T} T\left({ }^{\circ} C\right) \\
& P_{B}=P_{B_{o}}+\frac{\partial P}{\partial \varepsilon} \varepsilon(\mu \varepsilon)+\frac{\partial P}{\partial T} T\left({ }^{\circ} C\right)
\end{aligned}
$$

Therefore by monitoring both power and frequency simultaneously, temperature and strain can be determined. There are some limitations though. The spatial discrimination is determined by the time elapsed between the launching of the pump and receiving the Brillouin back-scattered signal therefore it typically takes several minutes to determine the strain location, Hotate (2003). The accuracy is also quite limited. Conventional DBS systems can achieve $\pm 4^{\circ}$ temperature accuracy and $\pm 100 \mu \varepsilon$ strain. With special polarization maintaining fibers higher accuracy of $\pm 1^{\circ}$ and $\pm 5 \mu \varepsilon$ has been reported, Bao (2006). Dynamic measurements using a correlation based continuous wave technique with data acquisition rates of up to $8 \mathrm{~Hz}$ and $\pm 35 \mu \varepsilon$ accuracy are also achievable but the temperature cross sensitivity of such system is not mentioned Hotate (2003). To conclude DBS systems offer true distributed sensing capabilities however due to limited spatial resolution and dynamic measurement limitations they are mainly suitable for large 
scale structural health monitoring such as on pipelines and bridge supports and their use is still quite limited for crack and vibration detection on small structures such as engines or airplane parts. The shape monitoring is also difficult with DBS systems since they are not directly sensitive to bending.

\subsubsection{Fiber Bragg Gratings}

Fiber Bragg gratings are a periodic structure written in the core of a fiber. They reflect a narrowband portion of incident light and transmit the rest. First to use FBG based fiber optic sensors for SHM were Morey et al. (1989). The temperature and strain sensing is done by measuring the wavelength shift of the center wavelength of the reflected or transmitted spectrum. The temperature sensitivity is typically around $10 \mathrm{pm} /{ }^{\circ} \mathrm{C}$, the strain sensitivity is $1 \mathrm{pm} / \mu \varepsilon, \mathrm{Xu}(1993 \mathrm{a})$. The sensitivity accuracy is mostly limited by interrogator resolution. With modern devices, with sub-picometer resolution the accuracies of $\pm 1 \mu \varepsilon$ and $\pm 0.1^{\circ} \mathrm{C}$ are achievable. Graver et al. (2004), provide a summary of civil applications, where FBGs were used to collect predetermined point strain data in bridges, support structures and dams. In all of the above applications FBGs were chosen for their reliability and ability to operate in harsh environments. The highlighted drawbacks are cost and difficulty of installation as well as the cost of interrogation equipment and data analysis. This stresses the need for simpler sensors with better application specific packaging. Some of the factors that might drive the cost down are the capability of multiplexing multiple sensors on the same fiber line. This will allow to spread the cost of the expensive interrogation equipment over many sensors, driving the overall cost down. 
Another important area where more progress needs to be done is temperature and strain discrimination. The most straight forward approach is to use a thermocouple or strain relieve package for one of the gratings in the grating array and use it to temperature compensate the rest of the FBGs, Kang et al. 2002, Wu et al. 2004 and Ben-Simon et al. 2007. However this often leads to bulky and costly systems which are difficult to interrogate. Other, more elaborate temperature compensation techniques are based on bonding a grating between two metals such that when the structure undergoes thermal expansion one of the metals would compress the grating and another would stretch it to compensate for the temperature sensitivity of the grating Yoffe et al., 1995. This technique is now a commercially available product, MicronOtics 2008. A more compact solution, suitable for integration is combining gratings with significantly different temperature and strain sensitivities. Guan et al., 2000 , have achieved $\pm 8.5 \mu \varepsilon$ and $\pm 1.6^{\circ} \mathrm{C}$ accuracy for a measurement range of $500 \mu \varepsilon$ and $100^{\circ} \mathrm{C}$ by splicing two fibers with different doping concentrations and writing a grating over the splice point. Shu et al., 2002, has reported on the dependence of the temperature sensitivity on the grating fabrication and hydrogen loading, which also allows to discriminate between the two parameters. But so far the most attractive technique has been the use of grating pairs written under exactly the same conditions and in the same fiber but with different center wavelengths and consequently different sensitivities. The gratings can even be written on the same spot to provide collocated temperature and strain readings. Xu et al., 1994b was the first one to report on the use of a FBG grating pair with a large wavelength separation in the range of 850 to $1300 \mathrm{~nm}$. They achieved $\pm 5^{\circ} \mathrm{C}$ and $\pm 10 \mu \varepsilon$ in a measurement range 
of $50^{\circ} \mathrm{C}$ and $600 \mu \varepsilon$. In a more recent publication Caucheteur et al., 2005b have achieved $\pm 3^{\circ} \mathrm{C}$ and $\pm 12 \mu \varepsilon$ in a measurement range of $25-85^{\circ} \mathrm{C}$ and $0-1500 \mu \varepsilon$ range for a dual grating pair with the wavelengths ranging from 1534 to $1579 \mathrm{~nm}$. A good review of the dual grating techniques has been done by Shu et al., 2002. The biggest problem which has been identified with dual grating techniques is that they require over $100 \mathrm{~nm}$ of bandwidth and therefore reduce the multiplexing capability of the system. To date there are no reports of a temperature compensated dual grating system that can be easily multiplexed.

The other issue which is common for FBGs is slightly non-linear temperature sensitivity. For large temperature ranges over $100^{\circ} \mathrm{C}$ non-linearity related temperature errors can be as much as 3\% Flockhart et al., 2004. However it can be used to a certain advantage since the temperature sensitivity of FBGs decreases significantly for low temperatures. James at al., 2002, have compared the response of FBGs and resistive foil strain gauges at temperatures down to $2.2 \mathrm{~K}$. They concluded that due to their zero temperature sensitivity and electromagnetic immunity FBGs are better suited to structural monitoring of superconducting magnet support structures, storage or transport vessels for cryogens, liquid hydrogen fuel tanks and particle physics experiments than conventional electrical gauges. Zhang et al., 2006, has also demonstrated the use of FBGs at cryogenic temperatures, they confirmed that temperature sensitivity does indeed go to zero at low temperatures but also showed that the strain sensitivity actually increases slightly for low temperatures, further emphasising the need for temperature and strain discrimination. 
High acquisition rates are also important for such techniques as modal analysis. FBG systems have been reported to perform modal analysis and retrieve modal shapes in good agreement with the reference sensors up to a frequency of $1.5 \mathrm{kHz}$. A dynamic resolution of $40 \mathrm{n} \varepsilon /(\mathrm{Hz})^{1 / 2} @ 50 \mathrm{KHz}$ has been achieved Capoluongo et al., 2007. The dynamic response of FBG sensors is also mainly limited by the interrogator. Interrogators with moving parts such as Fabry Perot can achieve data acquisition rates of $100 \mathrm{~Hz}$ while intensity based interrogators such as Mach Zender interferometer or Arrayed Waveguide Grating are capable of $\mathrm{MHz}$ acquisition speeds Hongo et al., 2005.

\subsubsection{Other Techniques}

Various interferometric sensors such as the ones based on Fabry-Perot cavities have also been tried. Wang et al., 2007, have reported on the use of Fabry-Perot cavities created between pairs of FBGs. They showed that up to 56 sensors can be multiplexed within the C-band. Strain isolated gratings were required though to compensate for the temperature.

Another parameter which is particularly difficult to detect with fiber optic sensors is bending. Intrinsically regular FBGs are not sensitive to bending. However, the use of specialty multicore fibers Fender et al., 2006 or surface relief gratings Lowder et al., 2006 does allow to detect bending. The disadvantage is the cost and difficulty associated with the use and handling of multicore fibers. Long period gratings have also been extensively explored for temperature compensated bend measurements. LPGs couple light to forward propagating cladding modes which have very distinct temperature, strain and bending wavelength and power sensitivities Liu et al., 2000, Rao et al., 2003. The problem with 
long period gratings is that the resonances are typically tens of nm wide which again makes it difficult to multiplex a large number of sensors.

The last technique which has only appeared in the past few years is tilted FBGs. As the name suggests the grating planes are tilted which like in LPGs allows to couple light to cladding modes however, the difference is that the coupling is backward and the resonances are much narrower. The bend sensitivity of the cladding modes of a TFBG has been addressed in a number of publications. Back et al., 2002 were first to explore the power variation of the first cladding mode known as the ghost mode, they were also first to note that the bending sensitivity depends on the direction of bending. Caucheteur et al., $2005 \mathrm{~b}$, integrated the area of all of the cladding modes and correlated it to bending. They demonstrated that the bending sensitivity is temperature independent however it is nonlinear and it would be difficult to multiplex such sensor as the cladding modes take up over $20 \mathrm{~nm}$ of bandwidth. Finally, Chen et al., 2006 has reported on the differential temperature/strain sensitivity of the cladding modes however again the discrimination is possible only for the modes that are far apart from each other.

\subsection{Summary}

To conclude fiber-optic sensors are one of the most promising solutions for complete structural health monitoring however there is still a lot of work remaining to be done in temperature and strain discrimination, multiplexing and reducing the system complexity. Brillion scattering can successfully tackle the temperature and strain discrimination as well as offer true distributed sensing capability however the dynamic response is limited. FBG based sensors can be multiplexed and can be very fast but temperature and strain 
discrimination is limiting their use. In the rest of this thesis I will present experimental results on multi-parameter sensing using the simplest configuration of dual FBGs with center wavelengths inside a $\mathrm{C}$-band. Then the more complex but potentially more promising tilted FBGs will be explored. 


\section{Chapter 3: Fiber Bragg Grating Theory}

\subsection{Introduction to Fiber Bragg Gratings}

The fiber Bragg grating, in its most simple form can be defined as a periodic modulation of the index of refraction along the fiber core. The first FBGs have been demonstrated by Hill et al., 1987, by setting up a standing wave pattern of UV light in the fiber core. The germanium doped core of the fiber is intrinsically photosensitive to UV light and therefore a standing UV wave is capable of inducing a permanent, periodic refractive index perturbation. Modern gratings are typically written in hydrogen loaded fibers in order to increase the photosensitivity Lemaire et al., 1993, and using a phase mask technique first proposed by Hill et al., 1993. Once the grating is written it acts as a narrow band wavelength selective filter. That is, if a broadband light source is coupled into the grating a notch will appear in transmission and a narrow peak will be reflected back. The center wavelength of that peak depends on the periodicity of the refractive index modulation and the tilt of the grating planes, and can be described by the Bragg condition:

$$
\lambda_{B}=\frac{2 n_{e f f} \Lambda_{g}}{\cos \theta}
$$


where $n_{\text {eff }}$ is the effective index of refraction of the single core mode of the fiber, $\Lambda_{\mathrm{g}}$ is the period of the grating and $\theta$ is the tilt angle of the grating planes as shown in Figure 3.1 .

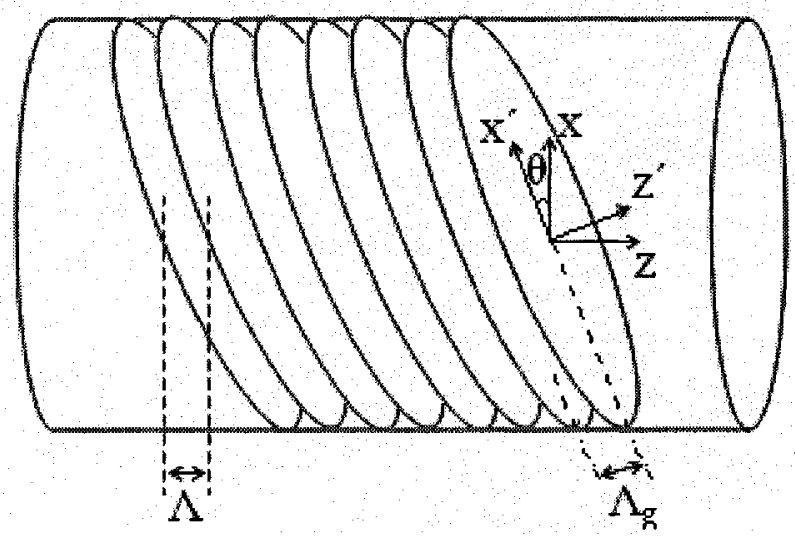

Figure 3.1: Tilted Fiber Bragg grating plane orientation

In most applications the tilt angle is zero in which case the transmission spectrum of such grating will have a single notch and the reflection spectrum will have a single peak. However there are cases when grating planes are tilted, which results in coupling into cladding modes. In the remainder of the chapter, first the wavelength sensitivity of the zero degree Bragg grating to strain, temperature and pressure will be discussed and then some background on the dual grating temperature and strain discrimination concept will be provided. The last section will deal with tilted gratings, their spectral shape and the coupling strength sensitivity of the cladding modes to bending.

\subsection{Straight FBG Sensitivity}

If strain, temperature or pressure are applied to the fiber the effective refractive index of the fiber and the period of the grating change. From equation 3.1 it can be seen that 
such changes will cause a shift in the Bragg wavelength of a grating as expressed in equation 3.2. Othonos, 1999, Xu, 1994.

$$
\frac{\Delta \lambda_{B}}{\lambda_{B}}=\left(\frac{1}{n_{e f f}} \frac{\partial n_{e f f}}{\partial l}+\frac{1}{\Lambda} \frac{\partial \Lambda}{\partial l}\right) \Delta l+\left(\frac{1}{n_{e f f}} \frac{\partial n_{e f f}}{\partial T}+\frac{1}{\Lambda} \frac{\partial \Lambda}{\partial T}\right) \Delta T+\left(\frac{1}{n_{e f f}} \frac{\partial n_{e f f}}{\partial P}+\frac{1}{\Lambda} \frac{\partial \Lambda}{\partial P}\right) \Delta P
$$

The first term is the strain sensitivity, the second term is the temperature sensitivity and the third term is the pressure sensitivity. The following sections will describe each of the above terms in detail.

\subsubsection{Sensitivity to Strain}

The first term of equation 3.2 describes the FBG peak center wavelength shift due to applied elongation. The term can be rearranged into a more useful form as follows:

$$
\frac{\Delta \lambda_{B}}{\lambda_{B}}=\left(\frac{\partial n_{\text {eff }}}{n_{\text {eff }}} \frac{l}{\partial l}+\frac{\partial \Lambda}{\Lambda} \frac{l}{\partial l}\right) \frac{\Delta l}{l}
$$

Where $\frac{\partial \Lambda}{\Lambda}=\frac{\Delta l}{l}=\varepsilon_{a x}$, the axial strain, substituting into equation 3.3

$$
\frac{\Delta \lambda_{B}}{\lambda_{B}}=\left(1+\frac{1}{\varepsilon_{a x}} \frac{\partial n_{e f f}}{n_{e f f}}\right) \varepsilon_{a x}
$$

Where $-\frac{1}{\varepsilon_{a x}} \frac{\partial n_{e f f}}{n_{e f f}}$ is commonly known as strain-optic coefficient, [Hei et al., 2002 given by:

$$
P_{e}=-\frac{1}{\varepsilon_{a x}} \frac{\partial n_{e f f}}{n_{e f f}}=\frac{n_{e f f}^{2}}{2}\left(P_{12}-v\left[P_{11}+P_{12}\right]\right)
$$


Where $P_{11}$ and $P_{12}$ are Pockel's coefficients of the strain-optic tensor, and $v$ is the Poisson's ratio. For a typical germano-silicate optical fiber $P_{11}=0.113, P_{12}=0.252, v=$ 0.16 and $\mathrm{n}_{\mathrm{eff}}=1.452$. Therefore from equation 3.4 the theoretical $\mathrm{P}_{\mathrm{e}}=0.204$. It then follows that the wavelength normalized strain sensitivity, $\frac{d \lambda}{\lambda d \varepsilon}$ (also known as gauge factor) $K_{\varepsilon}=1-P_{e}=0.796 \mu \varepsilon^{-1}$ Othonos and Kalli, 1999. It should be noted that the strain sensitivity will be wavelength dependent. It arises from the wavelength dependence of the effective refractive index. Using the Maltison 1965, model to account for the wavelength dependence of the refractive index the strain sensitivity for a broad spectral range is plotted in Figure 3.2.

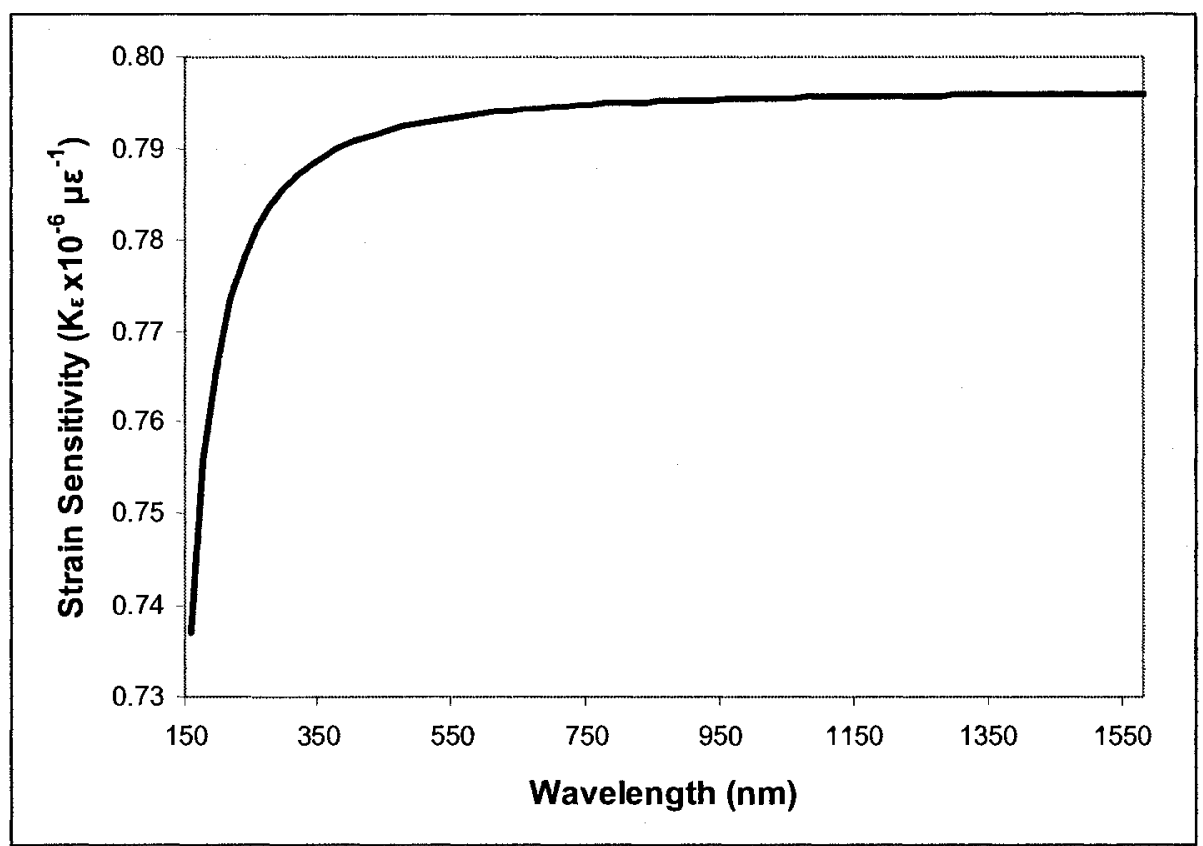

Figure 3.2: Expected Strain sensitivity of a FBG for a range of wavelengths

In most practical situations the grating is either bonded or embedded inside a material being monitored. When bonded, the physical sensitivity of a grating is not expected to 
change however if the strain transfer from a substrate to a grating is not perfect the sensitivity would be expected to be less. As an illustration a symmetrically embedded grating is shown in Figure 3.3.

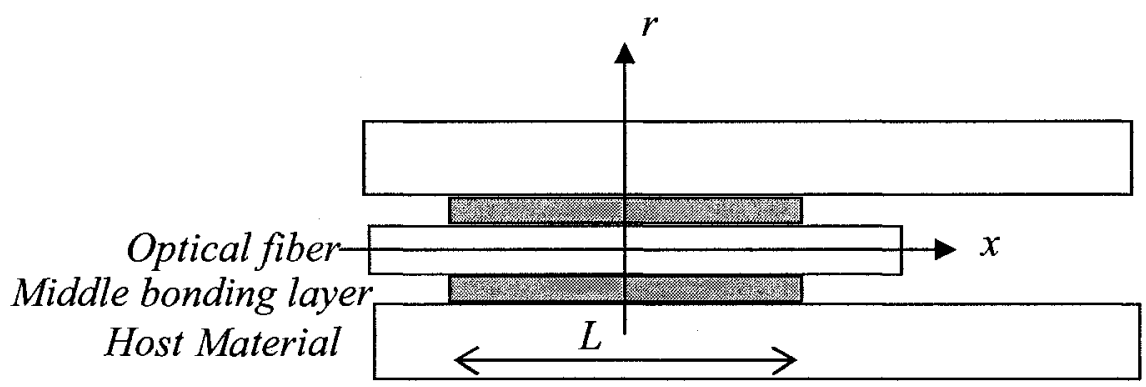

Figure 3.3: Fiber embedding configuration

The strain transfer depends on the geometry and material properties of the optical fiber coating and the middle layer, which is typically a polymer coating and epoxy type adhesive. If the material properties of the system are known then the actual strain in a substrate can be correlated to the strain seen by the grating via equation 3.6.

$$
\varepsilon_{g}(x)=\varepsilon_{m}\left[1-\frac{\cosh (k x)}{\cosh (k L)}\right]
$$

Where $x$ is the axial position along the grating $\varepsilon_{g}$ is the strain in the fiber, $\varepsilon_{m}$ is the strain in the host material, $L$ bonded gauge length and $k$ is the strain lag parameter defined in equation 3.7. Li et al., 2006.

$$
k^{2}=\frac{1}{(1+\mu)\left(E_{g} / E_{c}\right) r_{g}^{2} \ln \left(r_{m} / r_{g}\right)}
$$


Where $\mu$ is the poisons ratio $E_{g}$ is the fiber Young modulus, $E_{c}$ is the middle layer Young modulus, $r_{g}$ is the fiber radius and $r_{m}$ is the inner radius of the host material. As shown by Li et al., 2006, longer gauge lengths and thinner coatings with higher Young moduli will ensure better strain transfer to the core of the fiber.

\subsubsection{Sensitivity to Temperature}

The temperature sensitivity of FBGs is described by the second term of the equation 3.2. It reflects two effects; the change in grating spacing due to thermal expansion and temperature-dependent variation of the refractive index. For simplicity it can be rewritten as shown in equation 3.8:

$$
\Delta \lambda_{B}=\lambda_{B}\left(\alpha_{\Lambda}+\alpha_{n}\right) \Delta T
$$

where $\alpha_{\Lambda}=(1 / \Lambda)(\partial \Lambda / \partial T)$ is the thermal expansion coefficient for the silica fiber $\left(0.55 \times 10^{-6}\right.$ for silica $)$ and $\alpha_{n}=\left(1 / n_{e f f}\right)\left(\partial n_{e f f} / \partial T\right)$ represents the thermo-optic coefficient; Othonos, 1999, Jewell, 1991, Magne, 1997. However there is also a number of publications which define thermo-optic coefficient as simply $\left(\partial n_{\text {eff }} / \partial T\right)$ Leviton, 2006, Maltison, 1965, Gosh, 1994. Due to this discrepancy in notation and quite a range of values for thermo-optic coefficient they have been summarized in Table 3.1 where units of $p p m / K$ represent $\left(1 / n_{e f f}\right)\left(\partial n_{e f f} / \partial T\right)$ and $/ K$ represent $\left(\partial n_{e f f} / \partial T\right)$. 


\begin{tabular}{|c|c|c|c|c|}
\hline $\begin{array}{l}\text { Thermo-optic } \\
\text { coefficient }\end{array}$ & Reference & $\begin{array}{l}\text { Wavelength } \\
(\mathrm{nm})\end{array}$ & $\begin{array}{l}\text { Temperature } \\
(K)\end{array}$ & Glass type \\
\hline $8.45 \times 10^{-6} / \mathrm{K}$ & Leviton, 2006 & 1550 & 295 & $\begin{array}{l}\text { Corning } 7980 \\
\text { fused silica }\end{array}$ \\
\hline $11 \times 10^{-6} / \mathrm{K}$ & Maltison, 1965 & 1550 & 298 & $\begin{array}{l}\text { General Electric } \\
\text { type } 151\end{array}$ \\
\hline $8.66 \mathrm{ppm} / \mathrm{K}$ & Jewell, 1991 & $\begin{array}{l}\text { Sodium D } \\
\text { line }\end{array}$ & 298 & SRM-739 Silica \\
\hline $7 \mathrm{ppm} / \mathrm{K}$ & Magne, 1997 & 1550 & 298 & NA \\
\hline
\end{tabular}

Table 3.1: Summary of thermo-optic coefficients of silica

A number of researchers also mention that the thermo-optic coefficient is non-linear which means non-linear temperature response of the FBG center wavelength is expected Flockhart et al., 2004, Leviton et al., 2006.

Finally the temperature sensitivity is wavelength dependent. This arises from the wavelength dependence of the effective refractive index and thermo-optic coefficient. Using the refractive index and thermo-optic coefficient data obtained by Leviton et al., 2006, the wavelength dependence of the temperature sensitivity is plotted in Figure 3.4.

From Figure 3.4 the wavelength normalized temperature sensitivity $d \lambda / \lambda d T$ of FBG with the center wavelength around $1550 \mathrm{~nm}$ is approximately $6.3 \times 10^{-6}{ }^{\circ} \mathrm{C}^{-1}$.

For bonded gratings the thermal expansion coefficient of the substrate must now be taken into the account and in the case of metals the thermal expansion term in equation 3.8 will be dominating the sensitivity. For example for 2024 aluminium the CTE is equal to $24.7 \mu \mathrm{m} / \mathrm{m}^{\circ} \mathrm{C}$ and therefore from equation 3.8 the temperature sensitivity will be expected to be around $30.52 \times 10^{-6 \circ} \mathrm{C}^{-1}$. The above would only be true if there would be a complete CTE transfer from the substrate to the grating. In reality, as shown in equation 3.6, certain losses are expected and the sensitivity would be less. 


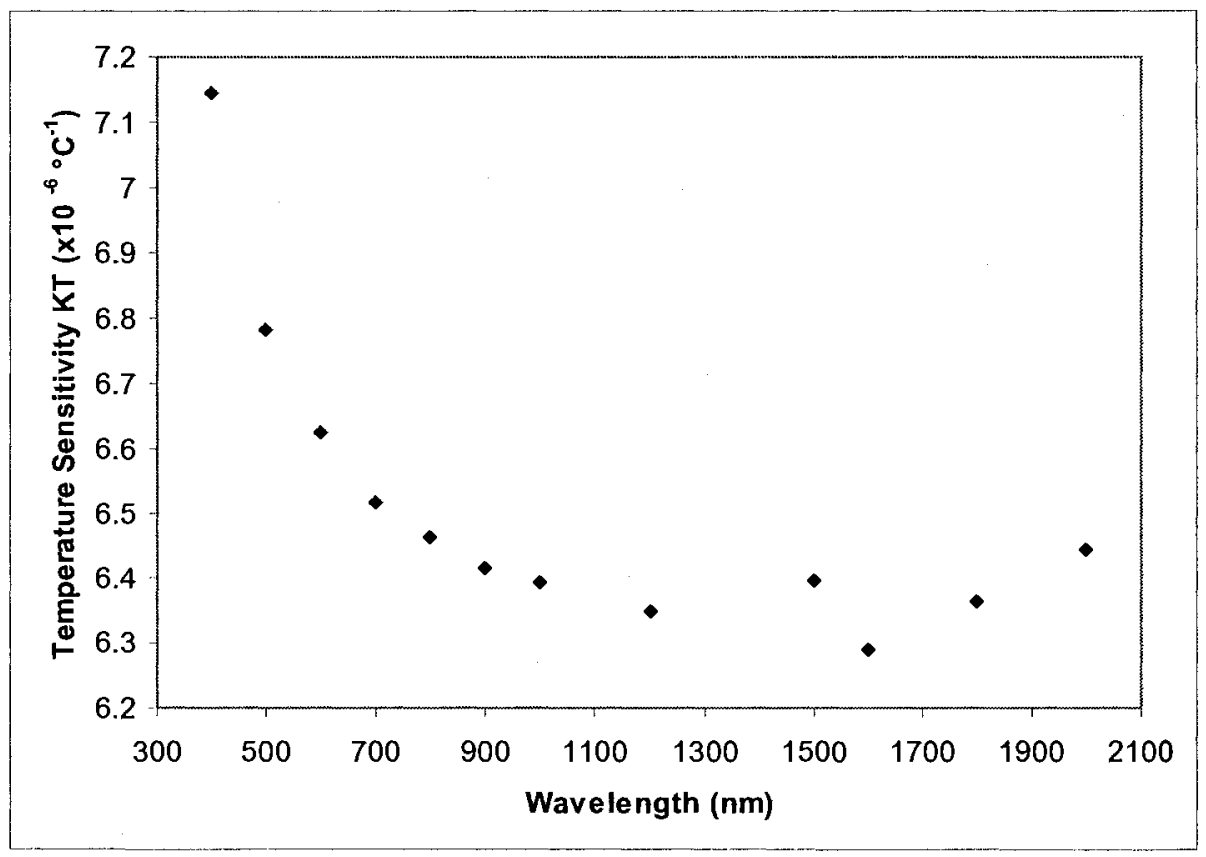

Figure 3.4: Wavelength dependence of the temperature sensitivity

\subsubsection{Sensitivity to Pressure}

The pressure sensitivity of FBGs is described by the third term of equation 3.2. Following the analysis in Xu et al., 1993, it can be rewritten in terms of the Young modulus $E$, Pockel's coefficients $P_{11}$ and $P_{12}$ and Poisson's ratio $v$ as shown in equation 3.9 .

$$
\frac{\Delta \lambda}{\lambda_{B}}=\left(-\frac{(1-2 v)}{E}+\frac{n_{e f f}^{2}}{2 E}(1-2 v)\left(2 P_{12}+P_{11}\right)\right) \Delta P
$$

The typical value for the isotropic pressure sensitivity is $3 \mathrm{pm} / \mathrm{MPa}$, in the scope of the structural health monitoring this value is low and can be neglected in most practical applications, Xu et al., 1993,. 


\subsubsection{Dual Grating Temperature-Strain Discrimination}

As has been shown in equation 3.2 fiber Bragg gratings are sensitive to temperature, strain and pressure. In section 3.2.3 it has been shown that the pressure sensitivity is negligible therefore in most cases it is important to discriminate between temperature and strain. From equations 3.4 and 3.8 it is apparent that both strain sensitivity $K_{\varepsilon}$ and temperature sensitivity $K_{T}$ are dependent on the effective refractive index $n_{e f f}$, which in turn is dependent on the wavelength of operation $\lambda$. Therefore for any two gratings with center wavelengths $\lambda_{1}$ and $\lambda_{2}$ the strain and temperature sensitivities $K_{T}$ and $K_{\varepsilon}$ are expected to be different. Now let $b=\Delta \lambda / \lambda_{B}$, using equation 3.2 the following sensitivity matrix can be built for two gratings with different center wavelengths:

$$
\left[\begin{array}{l}
b_{1} \\
b_{2}
\end{array}\right]=\left[\begin{array}{ll}
K_{r 1} & K_{\varepsilon 1} \\
K_{T 2} & K_{\varepsilon 2}
\end{array}\right]\left[\begin{array}{c}
\Delta T \\
\varepsilon
\end{array}\right]
$$

Rearranging and solving for $\Delta T$ and $\varepsilon$ :

$$
\left(\begin{array}{c}
\Delta T \\
\varepsilon
\end{array}\right)=\frac{1}{\left(K_{T 1} K_{\varepsilon 2}-K_{T 2} K_{\varepsilon 1}\right)}\left[\begin{array}{cc}
K_{\varepsilon 2} & -K_{\varepsilon 1} \\
-K_{T 2} & K_{T 1}
\end{array}\right]\left[\begin{array}{l}
b_{1} \\
b_{2}
\end{array}\right]
$$

From equation 3.11 it can then be noticed that knowing the relative wavelength shifts of the two gratings the temperature and strain can be discriminated effectively. The limitation is that the determinant of the matrix $K_{T 1} K_{\varepsilon 2}-K_{T 2} K_{\varepsilon 1}$, must be non-zero, that is the temperature and strain sensitivities of the two gratings must differ significantly. Otherwise a small error in $b$ will cause a large error in $\Delta T$ and $\varepsilon$. 
In general, a convenient way to quantify the error of the solution of a linear matrix is to calculate its' condition number. For any matrix of the form $\Omega x=b$ the relative error in $\mathrm{x}$ is given by equation 3.12 , Clifford, 1973:

$$
\frac{\|\delta x\|}{\|x\|} \leq K(\Omega) \frac{\|\delta b\|}{\|b\|}
$$

where $K(\Omega)$ is the condition number of the matrix $\Omega$. It can be seen that if the condition number is $K(\Omega) \gg>>1$ then a small error in b can lead to a rather large error in $\mathrm{x}$. In our case $x$ is either strain or temperature and $b$ is the relative wavelength shift. To keep the condition number small either strain or temperature sensitivity constants must differ significantly for the two selected wavelengths, as seen from equation 3.11 Flockhart et al., 2004 .

As mentioned in the previous chapter dual gratings have been explored extensively in the past, Table 3.2 presents the summary of the wavelength pairs used successfully.

\begin{tabular}{lllll}
\hline $\begin{array}{l}\text { Wavelength pair } \\
\lambda_{1}, \lambda_{2,}(\mathrm{~nm})\end{array}$ & Reference & $\begin{array}{l}K_{\varepsilon 1}, K_{\varepsilon 2} \\
\left(10^{-6} \mu \varepsilon^{-1}\right)\end{array}$ & $\begin{array}{l}K_{T 1,}, K_{T 2} \\
\left(10^{-6}{ }^{\circ} \mathrm{C}^{-1}\right)\end{array}$ & $K(\Omega)$ \\
\hline 848,1298 & Xu et al., 1994 & $0.70,0.74$ & $7.43,6.72$ & 123 \\
789,1561 & Brady et al., 1996 & $0.76,0.77$ & $8.37,7.61$ & 203 \\
660,1300 & $\begin{array}{l}\text { Sivanesan et al., } \\
\text { 2002 }\end{array}$ & NA & NA & 160.78 \\
780,1550 & $\begin{array}{l}\text { Sivanesan et al., } \\
\text { 2002 }\end{array}$ & NA & NA & 318.26 \\
1534,1579 & $\begin{array}{l}\text { Caucheteur et al., } \\
\text { 2004 }\end{array}$ & $0.80,0.82$ & $6.23,6.13$ & $1185^{*}$ \\
\hline
\end{tabular}

Table 3.2: Summary of dual grating pair condition numbers 
*Even with such a high condition number the authors were able to achieve the accuracies of $\pm 3^{\circ} \mathrm{C}$ and $\pm 12 \mu \varepsilon$ for simultaneous temperature and strain measurements, however only for a small temperature range $25-85^{\circ} \mathrm{C}$ and $0-1700 \mu \varepsilon$ range.

Table 3.2 confirms that in order to keep the condition number $K(\Omega)$ low and consequently reduce the sensitivity of temperature and strain to small errors in wavelength the wavelength separation of the two gratings must be large enough.

\subsection{Tilted Gratings, Cladding Modes}

The distinctive feature of tilted gratings TFBG is the presence of multiple notches corresponding to the cladding modes, which are only present in transmission. In reflection the cladding modes radiate out due to bend losses and coating absorption therefore only a single peak corresponding to the Bragg mode can be observed. Sample experimental TFBG transmission and reflection spectra are shown in Figure 3.5.

The Bragg center wavelength is given by equation 3.1 while the center wavelengths of the cladding modes are described by equation 3.13 Chen \& Albert, 2006.

$$
\lambda_{\text {cld }}^{i}=\left(n_{\text {cld }}^{i}+n_{\text {eff }}^{i}\right) \frac{\Lambda_{g}}{\cos \theta}
$$

where $n_{c l d}^{i}$ is the effective refractive index of a particular cladding mode and $n_{e f f}^{i}$ is the effective core refractive index at a particular cladding mode wavelength. The relative coupling strength of each mode is typically calculated using coupled mode theory, Erdogan, 1997. For the purpose of this work, commercially available Optigrating 4.2 grating simulation software has been used to simulate the TFBG spectrum. The fiber profile used in the simulations is described in Table 3.3. In the simulation the core was 
made photosensitive while the cladding was not. This means the grating was written in the core only.

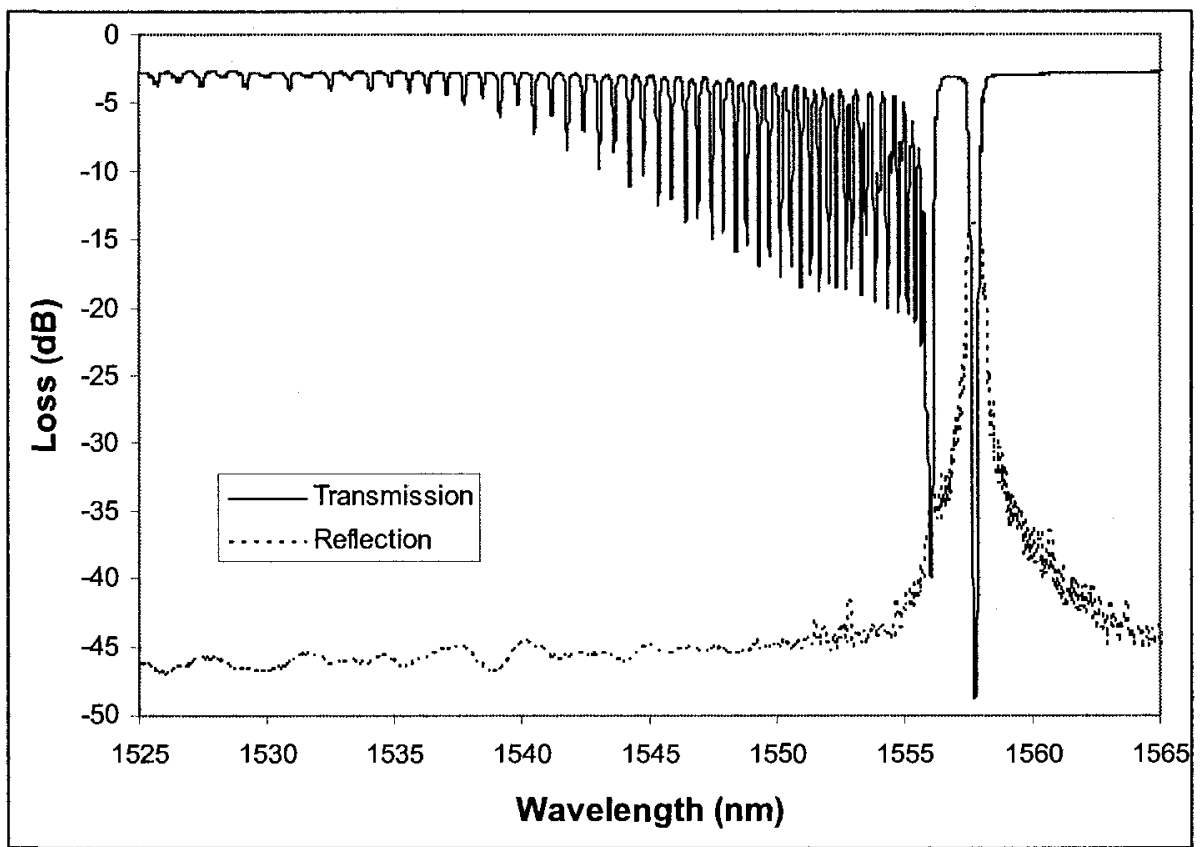

Figure 3.5: TFBG transmission and reflection spectrum for a $3^{\circ}$ tilted grating

\begin{tabular}{llll}
\hline Layer & $\begin{array}{l}\text { Radius } \\
(\mu \mathrm{m})\end{array}$ & $\begin{array}{l}\text { Index of } \\
\text { refraction }\end{array}$ & Photosensitivity \\
\hline Core & 4.15 & 1.4503 & 1 \\
Cladding & 62.5 & 1.444 & 0 \\
Air & 82.5 & 1 & 0 \\
\hline
\end{tabular}

Table 3.3: Fiber parameters used in the simulation

The simulated transmission and reflection spectra of the grating are shown in Figure 3.6. The grating was simulated with the plane spacing of $534 \mathrm{~nm}$ and a tilt of $3^{\circ}$. The effective refractive index of the single guided $\mathrm{LP}_{01}$ core mode (Bragg mode) was $n_{e f f}=1.4471$. In this work we are interested in how the spectrum evolves due to the change 
in physical parameters such as tilt and perturbations such as temperature, strain and bending.

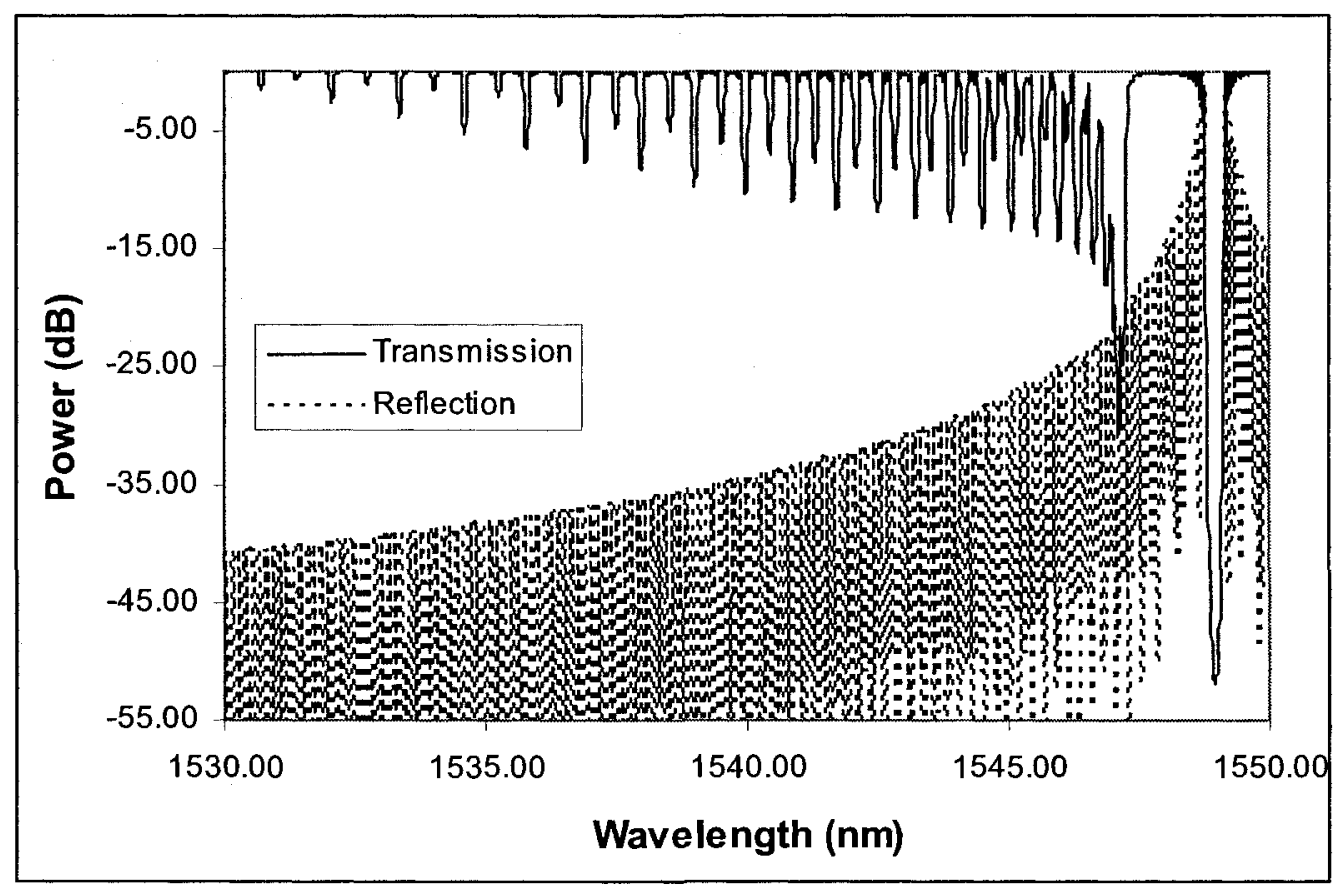

Figure 3.6: Optigrating transmission spectrum for a $3^{\circ} T F B G$

\subsubsection{Spectrum Sensitivity to Tilt.}

The tilt of the grating is a very important parameter which determines the coupling to a particular cladding mode. Figure 3.7 shows the simulated evolution for tilt angles ranging from $2^{\circ}$ to $5^{\circ}$. In general the coupling decreases significantly for higher tilt angles therefore in the following simulations the exposure time has been adjusted such that the ghost mode (first visible cladding mode) is approximately $30 \mathrm{~dB}$ for each tilt angle. The general trend that can be noticed from Figure 3.7 is that as the angle increases the relative coupling to the cladding modes increases while the coupling to the Bragg 
mode decreases. Thus by controlling the tilt of the grating the differential power between the cladding modes and the Bragg can be changed.
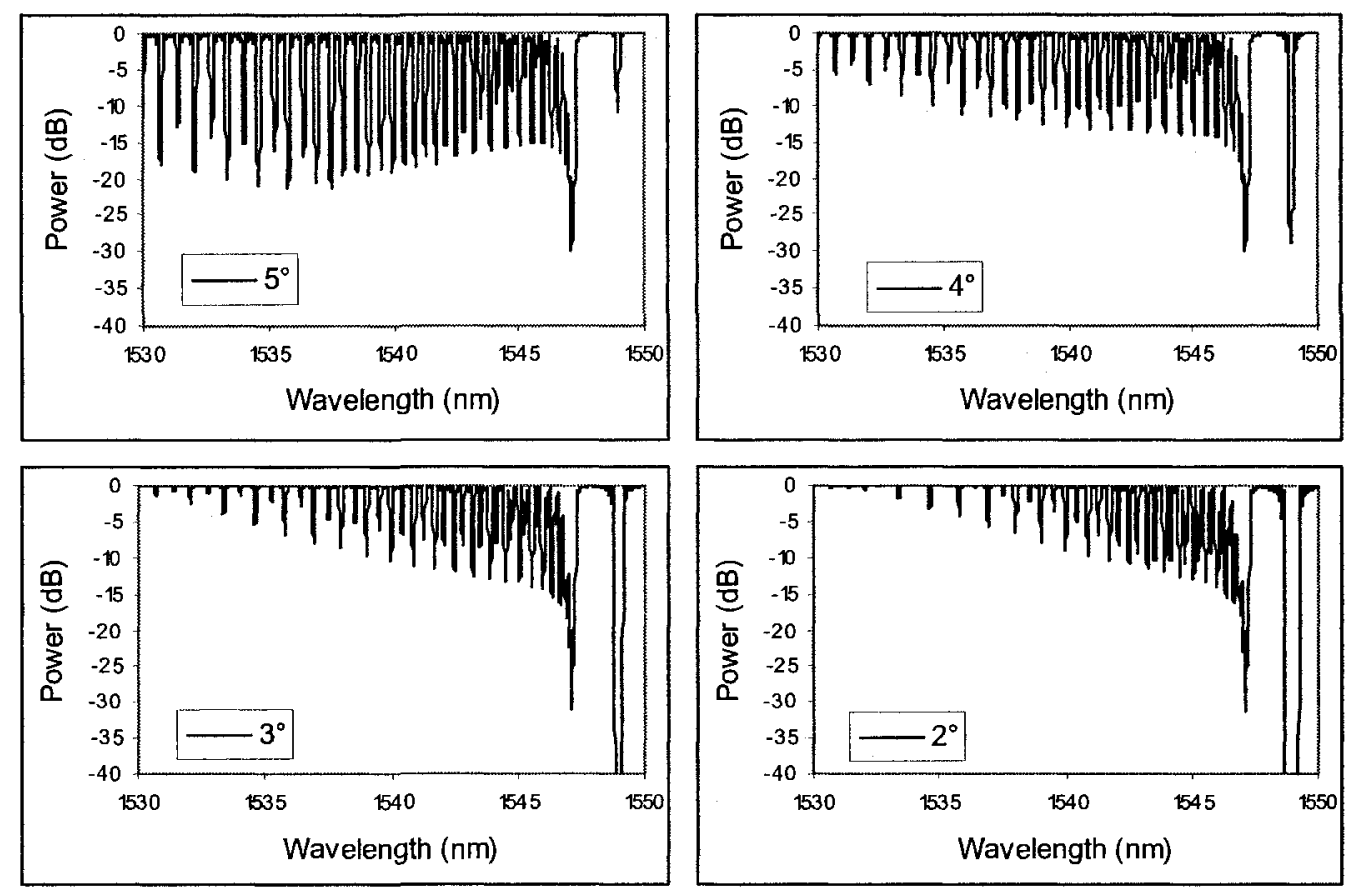

Figure 3.7:Grating spectrum evolution for various tilt angles

One mode that deserves special consideration is the ghost mode. Figure 3.8 shows the first few calculated cladding modes of a $5.7^{\circ}$ degree grating with the center wavelengths close to the ghost. As can be seen the first three modes are located very close to each other and in reality are hard to distinguish since they simply add up to one common distorted peak. It can also be noticed that the group is dominated by the modes represented with the dashed lines, which are corresponding to the $\mathrm{LP}_{11}-\mathrm{LP}_{14}$ modes.

The ghost mode can be very useful for sensing since it is the first cladding mode and therefore if the rest of the cladding modes can be filtered out the sensor would take up the least amount of bandwidth and it would be easier to multiplex multiple gratings on the 
same fiber. Figure 3.9 shows the coupling to a $\mathrm{Bragg}, \mathrm{LP}_{01}$ back reflection core mode and cladding modes $\left(\mathrm{LP}_{11}\right.$ through $\left.\mathrm{LP}_{14}\right)$ versus the grating tilt angle. It can be seen that for

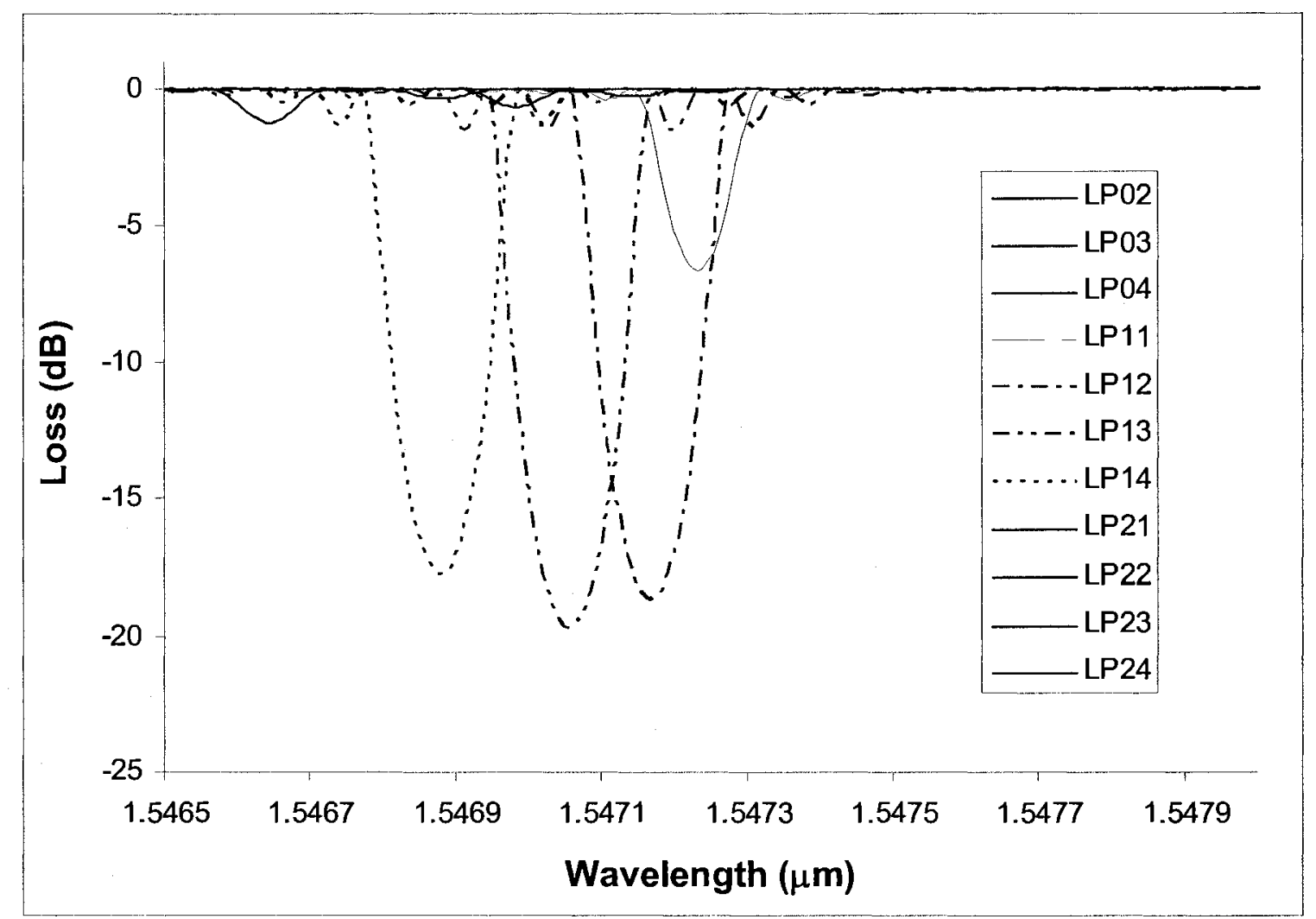

Figure 3.8: Break down of the modes which make up the ghost mode

angles less than $4.5^{\circ}$ the largest peak will correspond to the $\mathrm{Bragg} \mathrm{LP}_{01}$ mode, however for the tilt angles between $4.5^{\circ}$ and $6^{\circ}$ the Bragg rapidly decays and the largest peak will correspond to the Ghost mode dominated by the $\mathrm{LP}_{12}, \mathrm{LP}_{13}$ and $\mathrm{LP}_{14}$ cladding modes. Therefore by selecting a proper tilt angle the differential power between the ghost and the Bragg can be controlled very accurately and if required the Bragg could be eliminated all together leaving just the cladding modes. 


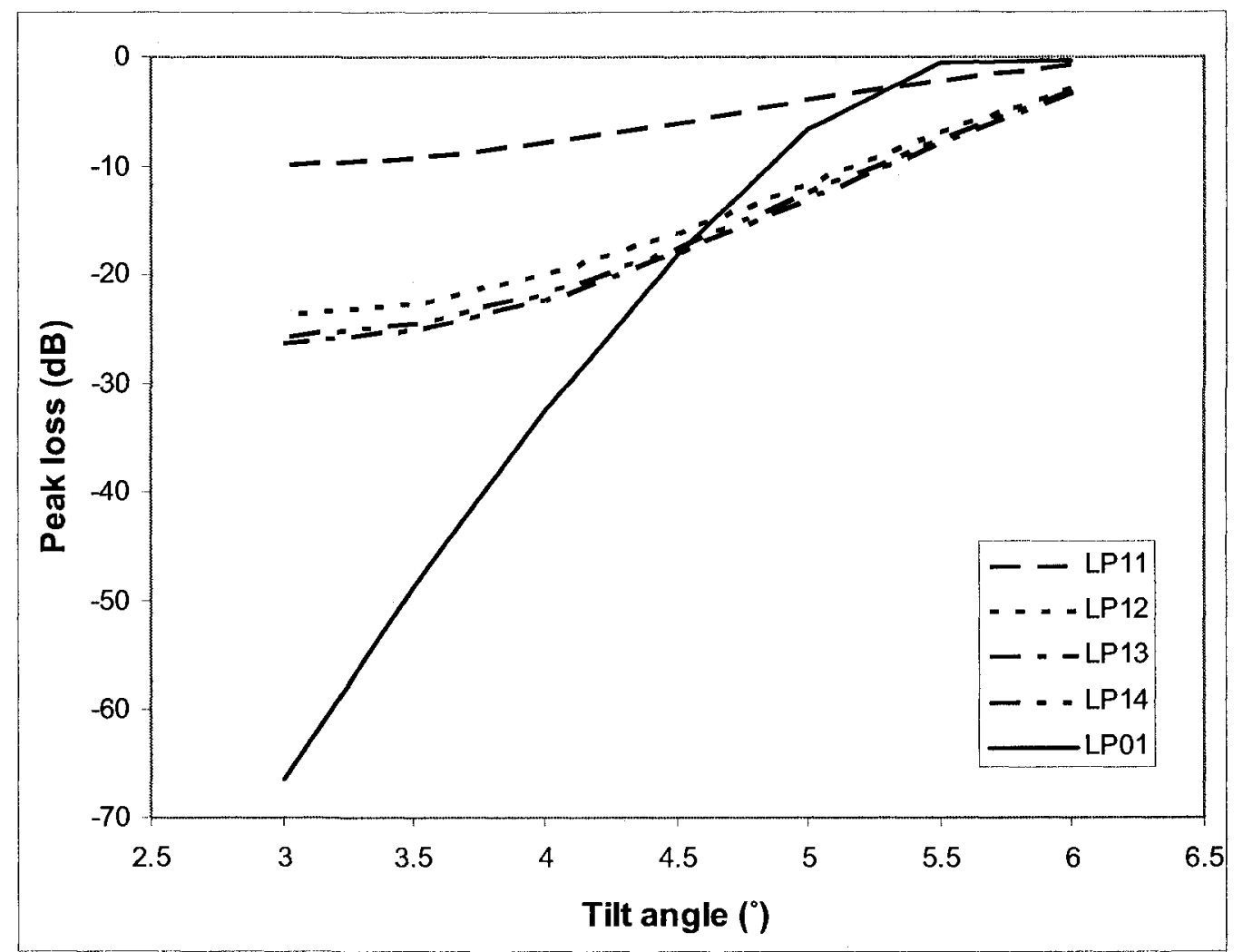

Figure 3.9: Peak loss for a selection of modes and tilt angles

\subsubsection{Sensitivity to Strain and Temperature}

As discussed earlier the strain and temperature only affect the period and the effective refractive index of the core mode. Therefore only the center wavelengths of the cladding modes and not the coupling coefficients are affected.

The wavelength sensitivity of the cladding modes to temperature is given by equation 3.14 .

$$
\Delta \lambda_{c l d}^{i}=\left(\frac{\left(n_{c l d}^{i}+n_{e f f}^{i}\right)}{\cos \theta} \frac{\partial \Lambda_{g}}{\partial T}+\frac{\Lambda_{g}}{\cos \theta} \frac{\partial\left(n_{c l d}^{i}+n_{e f f}^{i}\right)}{\partial T}\right) \Delta T
$$


As discussed in section 3.2.2 the $\partial \Lambda / \partial T$ is negligible compared to $\partial n / \partial T$ and inside the C-band the $\partial n / \partial T$ does not change appreciably, therefore the wavelength shifts of the cladding modes with temperature is expected to be the same as for the Bragg, especially for the low order cladding modes.

The strain sensitivity is given by equation 3.15 .

$$
\Delta \lambda_{c l d}^{i}=\lambda_{c l d}^{i}\left(1+\frac{1}{\left(n_{c l d}^{i}+n_{e f f}^{i}\right)} \frac{\partial\left(n_{c l d}^{i}+n_{e f f}^{i}\right)}{\partial \varepsilon}\right) \frac{\Delta l}{l}
$$

The term in the brackets $\frac{1}{\left(n_{\text {cld }}^{i}+n_{e f f}^{i}\right)} \frac{\partial\left(n_{c l d}^{i}+n_{e f f}^{i}\right)}{\partial \varepsilon}$ is typically defined as $-P_{e, c l d,}$ where $\partial n=0.5 n_{e f f}^{3}\left(P_{12}-v\left[P_{11}+P_{12}\right]\right) \delta \varepsilon \quad \partial n_{c l d} \approx \partial n_{e f f} \approx n_{S i}$ but $n_{\text {cld }}$ becomes appreciably less then $n_{e f f}$ for modes away from $\lambda_{B}$. For those cladding modes $P_{e, c l d}$ can then be approximated by $-\frac{1}{\left(n_{\text {cld }}^{i}+n_{\text {eff }}^{i}\right)} \frac{2 \partial\left(n_{S i}\right)}{\partial \varepsilon}$ which is slightly larger than $-\frac{1}{n_{e f f}} \frac{2 \partial\left(n_{S i}\right)}{\partial \varepsilon}=P_{B r a g g}$. Therefore since $\lambda_{B r a g g}>\lambda_{\text {cld }}$ the cladding modes are expected to have slightly lower strain sensitivity than the Bragg mode, however appreciable difference is only noticed for higher order cladding modes $10 \mathrm{~nm}$ away from the Bragg mode. This result has been confirmed experimentally by Chen and Albert, 2006.

\subsubsection{Sensitivity to Bending}

The bending of an initially straight fiber generates compressive strain on the inside of the fiber and tensile on the outside, see Figure 3.10. 


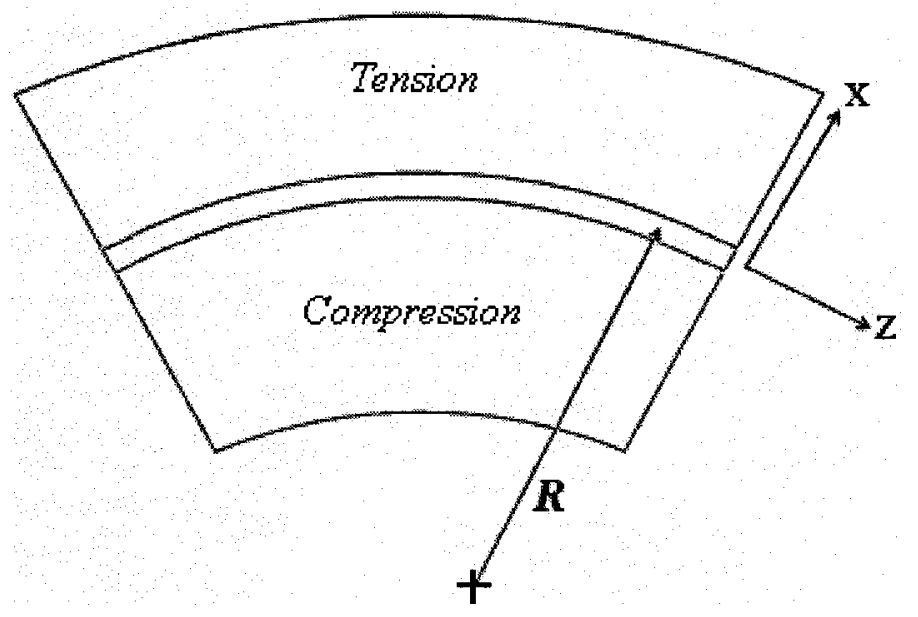

Figure 3.10: Stress distribution of a curved fiber

Through the photoelastic effect, this strain causes an index change for both $x$ - and $y$ polarization states equal to:

$$
\Delta n=-\frac{n^{3}}{2}\left[(1-v) P_{12}-v P_{11}\right] k x
$$

Where $\kappa$ is curvature (1/R), $v$ is the Poisson ratio, $P_{i j}$ are the photoelastic constants and $n$ is the refractive index of a particular mode Block et al., 2006. For a Silica fiber $\Delta \mathrm{n} \approx 0.31 \mathrm{kx}$. Fiber curvature and bend induced variations in the refractive index both tend to distort the mode field distributions, and ultimately push them away from the center of curvature Schermer et al., 2007. Therefore when determining the coupling to a particular cladding mode the overlap between the forward propagating core mode and the backward propagating cladding modes, the bend of a fiber must be considered. Moreover the refractive index change increases linearly with the distance from the center of the fiber. Then, to determine how bending will affect a particular mode the intensity profile of such mode must be taken into account. 


\begin{tabular}{|c|c|c|c|c|}
\hline $\mathrm{LP}_{01}$ & $\mathrm{LP}_{11}$ & $\mathrm{LP}_{12}$ & $\mathrm{LP}_{13}$ & $\mathrm{LP}_{14}$ \\
\hline$\Rightarrow$ & & 1 & t & $\frac{1}{2}$ \\
\hline
\end{tabular}

Figure 3.11: Mode intensity profiles

In this study we are mostly interested in the coupling between the forward propagating $\mathrm{LP}_{01}$ mode and the backward propagating $\mathrm{LP}_{01}, \mathrm{LP}_{11}, \mathrm{LP}_{12}, \mathrm{LP}_{13}$ and $\mathrm{LP}_{14}$ modes. As can be seen from Figure 3.11 the $\mathrm{LP}_{01}$ mode is tightly confined to the core, which means that the $\mathrm{LP}_{01}$-forward to $\mathrm{LP}_{01}$-backward coupling will not be affected much by the bending as the core of the fiber is located on the neutral axis. In contrast $\mathrm{LP}_{11}$, $\mathrm{LP}_{12}, \mathrm{LP}_{13}$ and $\mathrm{LP}_{14}$ modes do extend significantly into the cladding and therefore $\mathrm{LP}_{01^{-}}$ forward to $\mathrm{LP}_{11}, \mathrm{LP}_{12}, \mathrm{LP}_{13}$ and $\mathrm{LP}_{14}$-backward coupling will be significantly affected by the fiber bend. This makes it possible to detect the bend in the fiber even if the input power fluctuates. The level of the core Bragg mode can be used as a reference since it will not change due to bending and the level of a particular cladding mode can be used to monitor the bending. The advantage is that the differential measurements will not be susceptible to the light source instabilities.

\subsection{Summary}

In this chapter the theoretical background of zero degree and tilted FBGs have been introduced. The center wavelength shift of the core and cladding modes due to strain and temperature have been determined. It has been identified that the temperature sensitivity is slightly non-linear, which primarily limits the accuracy of the temperature 
measurements. The strain sensitivity is linear but when used as a bonded strain gauge the strain transfer between a substrate and core of a fiber must be considered. Temperature and strain discrimination is possible with dual wavelength gratings as long as the wavelength separation is large enough. Use of tilted gratings gives access to one more parameter: the power coupling to the cladding modes. Therefore, by monitoring the wavelength shift as well as the power; bending, temperature and strain applied to a fiber can be determined. In the next two chapters the experimental confirmation of the above and temperature compensated proof of concept tilted FBG sensors will be demonstrated. 


\section{Chapter 4: Free and Bonded Sensitivity and Temperature/Strain Discrimination Using Dual FBGs.}

\subsection{Introduction}

As has been described in Chapter 2: FBGs are very well suited for static and dynamic temperature and strain monitoring due to their simplicity. However one of the most significant challenges for fiber Bragg grating sensors is the temperature and strain cross sensitivity. In this chapter at first a number of different gratings will be characterized to temperature and strain, the experiments will be performed for both free and bonded gratings and a comparison will be made between the two cases. Then the ability to discriminate between temperature and strain will then be assessed.

\subsection{Temperature and Strain Sensitivity Characterization of Free FBGs.}

In this section the sensitivity to strain and temperature of free FBGs (not bonded or packaged in any kind of way) is characterized. The results are then grouped and the variations in sensitivity due to wavelength, coating and configuration are analyzed. The wavelengths available for testing were $1540 \mathrm{~nm}$ and $1560 \mathrm{~nm}$ single gratings and $1539 / 1545 \mathrm{~nm}$ dual gratings. The two coatings were acrylic and polyamide. And the two possible configurations were serial and collocated gratings. The FBGs were acquired from Avensys Inc. The acrylic coated gratings were written in a standard Corning, SMF- 
28 dual acrylate coated fiber with an outer coating diameter of $245 \mu \mathrm{m}$. Polyamide coated gratings were written in fibers manufactured by OFS and had a reduced coating diameter of $155 \mu \mathrm{m}$ for enhanced sensitivity. Both types of fibers were hydrogen loaded prior to grating inscription process.

The final two sections describe the combined temperature and strain sensitivity and the feasibility of temperature and strain discrimination using dual gratings.

\subsubsection{Strain Characterization Procedure and Results}

Strain characterization experimental set-up consisted of two metal posts separated by $\approx(800 \pm 2) \mathrm{mm}$, as shown in Figure 4.1 . One of the posts was bolted to the optical table and the other one was attached to a micrometer translational stage with a displacement accuracy of $\pm 0.005 \mathrm{~mm}$.

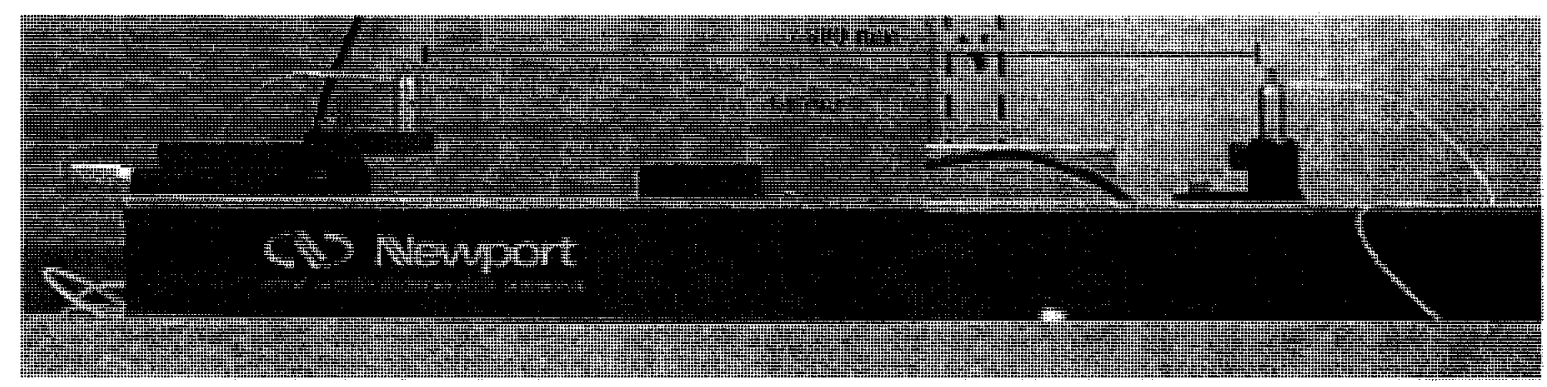

Figure 4.1: Strain characterization experimental set-up

At the attachment points the fiber was mechanically stripped of the protective coating and glued to the posts with Lepage 5 minute epoxy. After the epoxy was cured at room temperature for 24 hours the fiber was stretched in $0.15 \mathrm{~mm}$ increments up to a maximum elongation of $2.5 \mathrm{~mm}$ at room temperature. At every increment the transmission trace was 
taken with an Ando AQ6317B Optical Spectrum Analyzer. The resolution of the instrument was set to $0.05 \mathrm{~nm}$.

The peaks were identified using the average of the 3,4 and $5 \mathrm{~dB}$ bandwidth center wavelengths. The peak find procedure is as follows. First the minimum of the transmission spectrum was found, however due to the amplitude noise that point does not always correspond to the center wavelength of the peak. Therefore three lines 3,4 and 5 $\mathrm{dB}$ above the minimum were plotted as shown in Figure 4.2. Provided that the peak is symmetric, the average of the middle values between the intersections of the 3,4 and $5 \mathrm{~dB}$ lines and the raw data should give a more accurate peak center wavelength than simply taking the minimum.

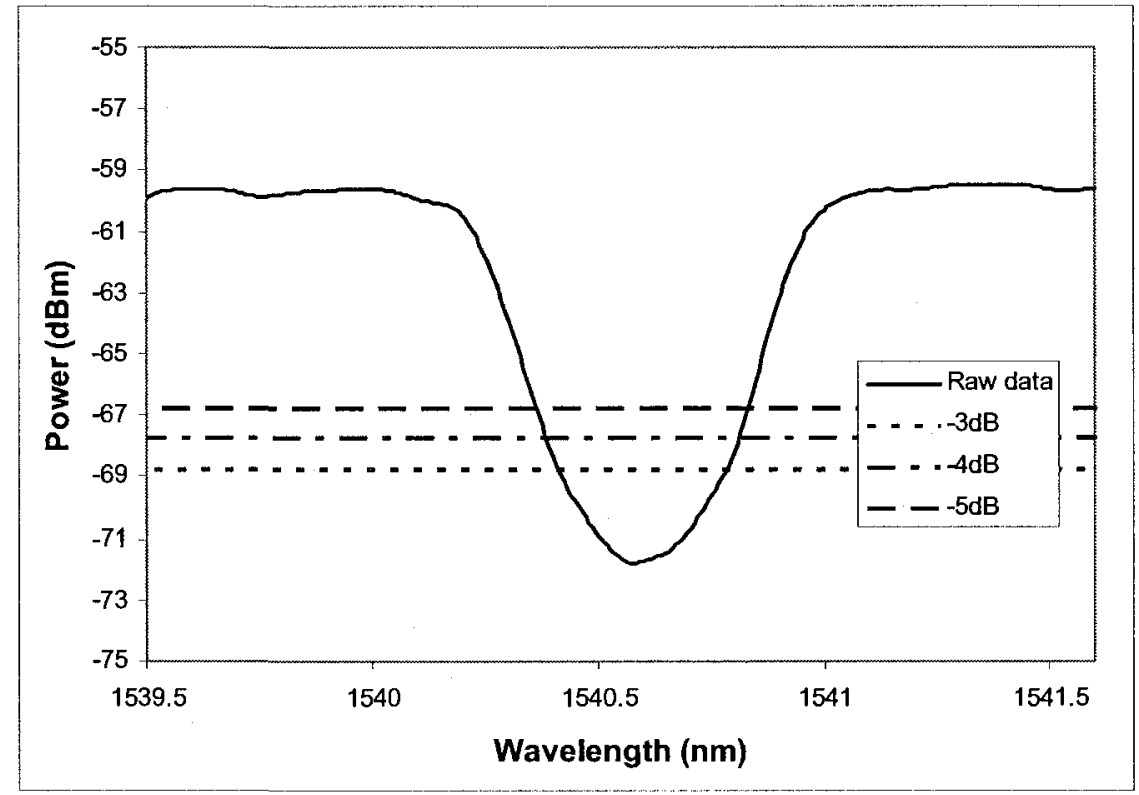

Figure 4.2: Typical transmission trace

The error in the described peak find algorithm was evaluated statistically by taking ten consecutive readings of the transmission trace of a typical FBG used in this 
experiment and calculating the standard deviation of the peak center wavelength calculated as explained earlier. The $99 \%$ confidence interval ( 3 standard deviations) was determined to be $\pm 2.7 \mathrm{pm}$. Therefore, for this work the accuracy of the interrogator is $\delta \lambda= \pm 2.7 \mathrm{pm}$.

The strain sensitivity was determined from the wavelength versus displacement plot as shown in Figure 4.3. To obtain the wavelength normalised strain sensitivity $K_{\varepsilon}$ the slope of the response in Figure 4.3 was multiplied by the unstrained length of the fiber containing the grating and then divided by the unstrained center wavelength.

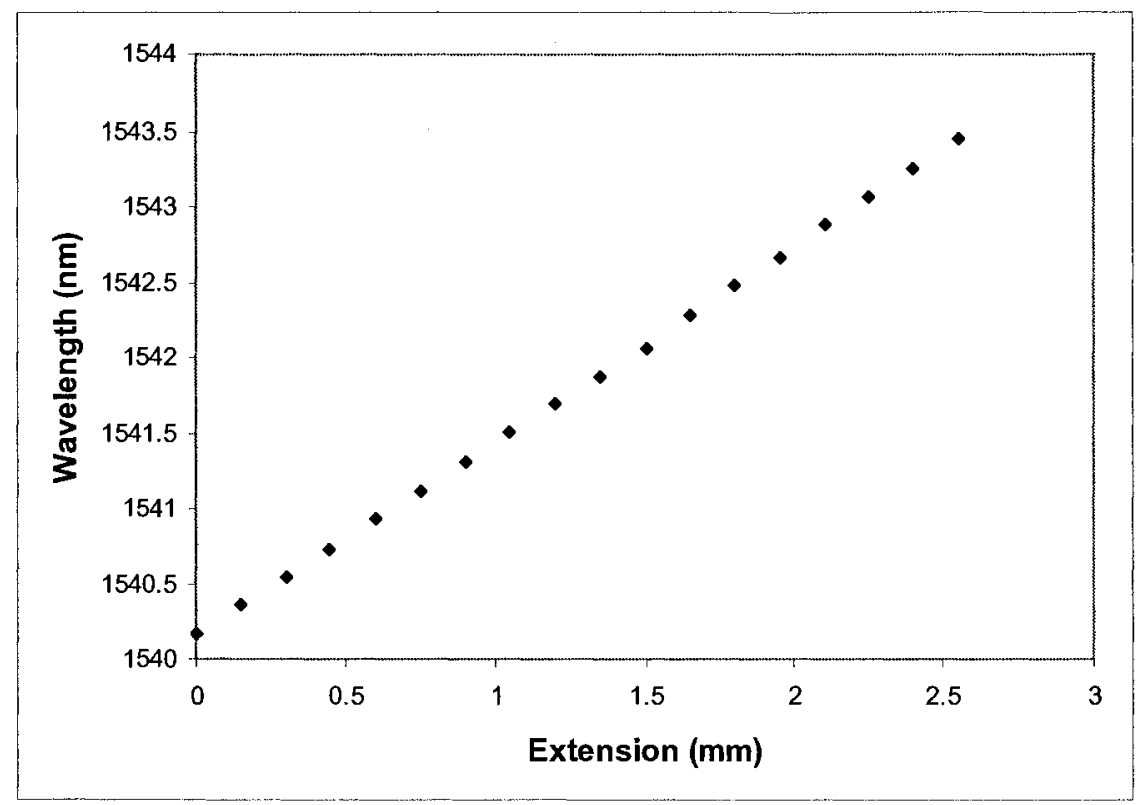

Figure 4.3: Typical extension vs. wavelength plot

The error on the obtained sensitivity can then be readily estimated using the standard error propagation as follows:

$$
\delta f(x, y, z \ldots)=\sqrt{\left(\frac{\partial f}{\partial x}\right)^{2}(\partial x)^{2}+\left(\frac{\partial f}{\partial y}\right)^{2}(\partial y)^{2}+\left(\frac{\partial f}{\partial z}\right)^{2}(\partial z)^{2} \ldots}
$$


The sensitivities obtained in various cases are presented in Table 4.1.

\begin{tabular}{lllll}
\hline Grating & Coating & Wavelength $(\mathbf{n m})$ & $\begin{array}{l}\boldsymbol{K}_{\varepsilon} \\
\left(10^{-6} \mu \varepsilon^{-1}\right)\end{array}$ & $\begin{array}{l} \pm \text { Error } \\
\left(10^{-6} \mu \varepsilon^{-1}\right)\end{array}$ \\
\hline A-11-13 & Acrylic & 1540.134 & 0.7944 & 0.0034 \\
A-a1-13 & Acrylic & 1540.139 & 0.7934 & 0.0028 \\
A-a2-10 & Acrylic & 1560.149 & 0.7927 & 0.0019 \\
A-p1-02 & Polyamide & 1540.084 & 0.8010 & 0.0019 \\
A-p2-09 & Polyamide & 1560.026 & 0.7988 & 0.0021 \\
A-p2-09 & Polyamide & 1560.013 & 0.7972 & 0.0022 \\
A-a1-12 & No Coating & 1540.166 & 0.7939 & 0.0028 \\
D-I-pa-08 & & 1539.101 & 0.7985 & 0.0020 \\
Dual Serial & Polyamide & 1544.949 & 0.8003 & 0.0020 \\
& & 1539.222 & 0.7942 & 0.0019 \\
D-II-pc-14 & & 1544.727 & 0.7954 & 0.0019 \\
Dual Collocated & Polyamide & & & \\
\hline
\end{tabular}

Table 4.1: Strain sensitivity characterization results

As was shown in section 3.2.1 it is expected that the wavelength normalized strain sensitivity in the $\mathrm{C}$-band is almost constant and equal to $0.796 \times 10^{-6} \mu \varepsilon^{-1}$. Previously reported results range from 0.7 to $0.82 \times 10^{-6} \mu \varepsilon^{-1}$ and are summarized in Table 4.2. The results produced in this work range from $0.793 \times 10^{-6} \mu \varepsilon^{-1}$ to $0.801 \times 10^{-6} \mu \varepsilon^{-1}$. The error on the characterization constant ranges from $\pm 0.002 \times 10^{-6} \mu \varepsilon^{-1}$ to $\pm 0.0034 \times 10^{-6} \mu \varepsilon^{-1}$. The main source of error is the uncertainty in the overall length of the fiber under test. 


\begin{tabular}{lll}
\hline Reference & Wavelength $(\mathrm{nm})$ & $\boldsymbol{K}_{\varepsilon}\left(10^{-6} \mu \varepsilon^{-1}\right)$ \\
\hline [Yoffe et al., 1995] & 1519.5 & 0.7 \\
[Hjelme et al., 1997] & 1527 & 0.70 \\
[Flockhart et al., 2004] & 1524 & 0.8062 \\
[Flockhart et al., 2004] & 1548 & 0.8076 \\
[Flockhart et al., 2004] & 1572 & 0.8073 \\
[Brady et al., 1996] & 1561 & 0.77 \\
[Caucheteur et al., 2004] & 1534 & 0.80 \\
[Caucheteur et al., 2004] & 1579 & 0.82 \\
\hline
\end{tabular}

Table 4.2: Previously reported strain sensitivity

\section{Strain Sensitivity Dependence on The Grating Center Wavelength:}

From Figure 4.4 and Figure 4.5 it can be observed that the wavelength dependence of the strain sensitivity has been observed only for the dual gratings.

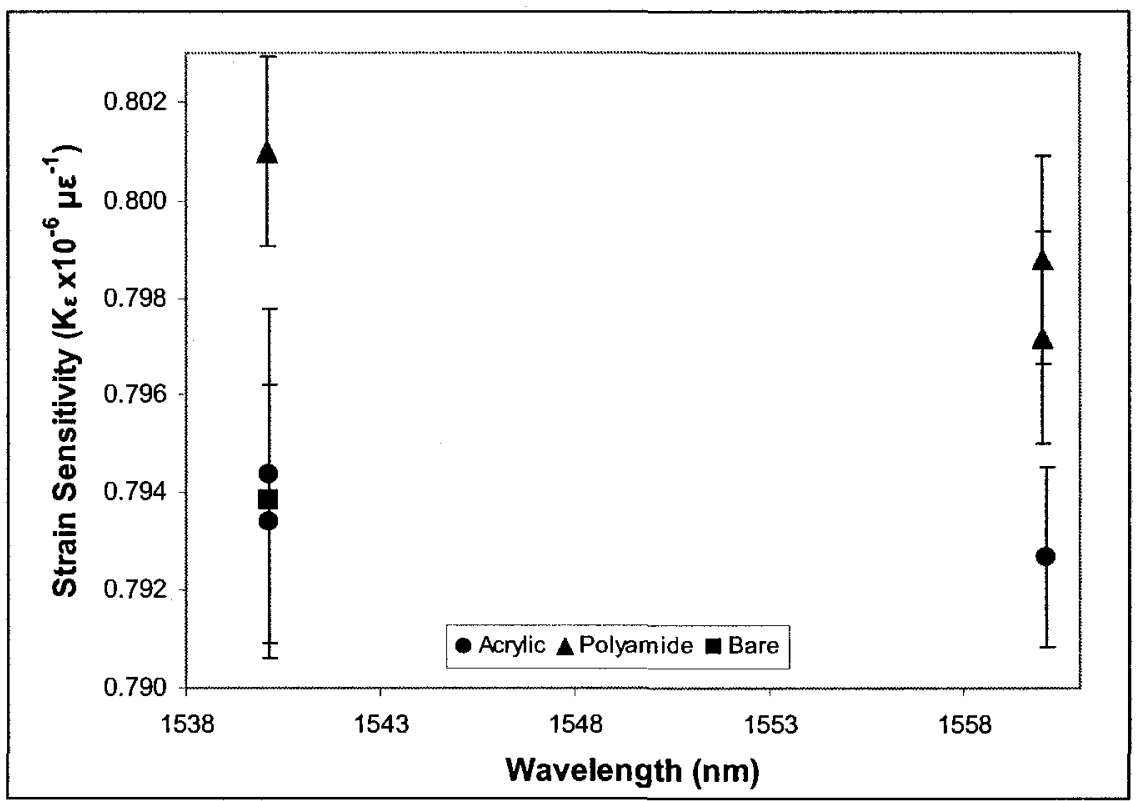

Figure 4.4: Strain sensitivity vs. wavelength for single gratings 
The increase is $0.00024 \times 10^{-6} \mu \varepsilon^{-1} \mathrm{~nm}^{-1}$ and $0.00036 \times 10^{-6} \mu \varepsilon^{-1} \mathrm{~nm}^{-1}$ for collocated and serial gratings respectively. However the increase is less then the error bars and was not observed for the single gratings. The possible reason for this trend appearing only for the dual gratings is because dual gratings are written in the same fiber and are tested at the same time and thus are not subject to the experiment repeatability errors. Therefore it can be concluded that higher wavelengths are more sensitive to strain however the effect is too small to be measured reliably and therefore is negligible in the C-band.

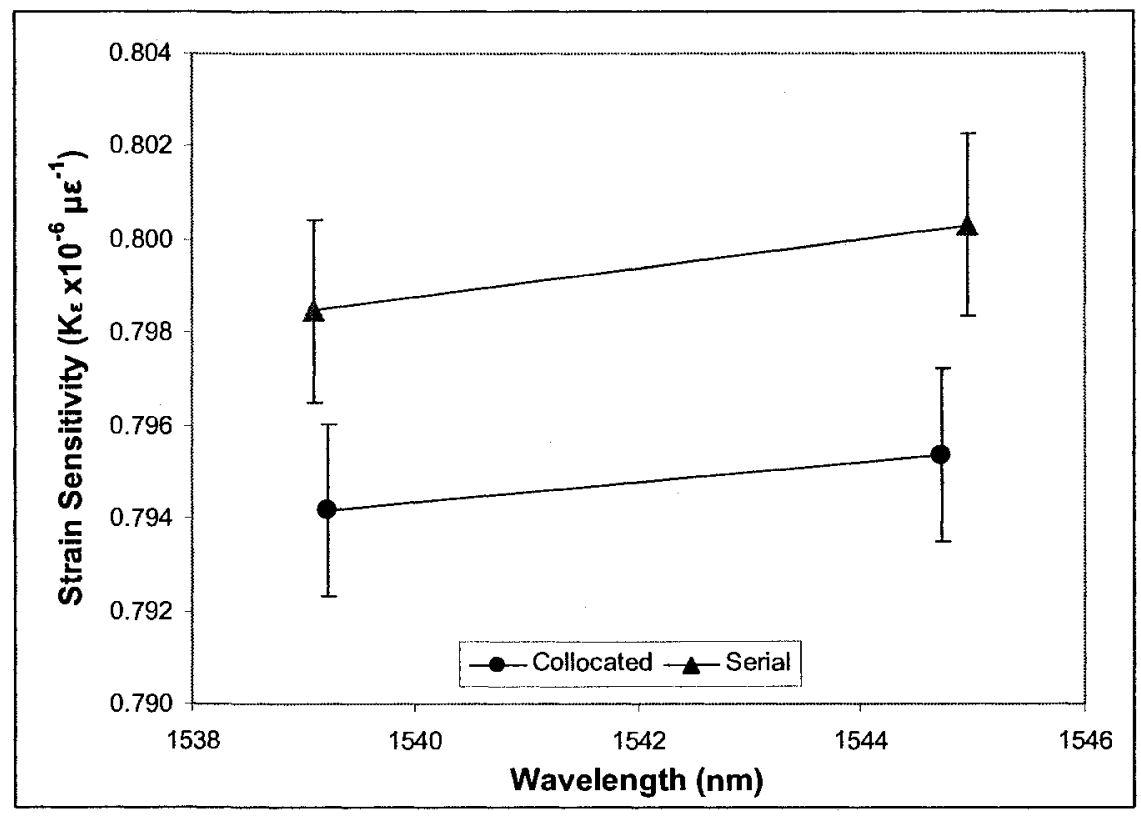

Figure 4.5: Strain sensitivity vs. wavelength for dual gratings

\section{Strain Sensitivity Dependence on The Coating.}

From Figure 4.4 it can also be observed that acrylic coated fiber Bragg gratings have lower strain sensitivity then polyamide. The maximum difference is $0.006 \mu \varepsilon^{-1}$ the discrepancy can be attributed to the different physical properties of acrylic and polyamide coated fibers because they come from different manufacturers. This conclusion is further 
supported by the fact that a bare FBG, which was prepared by mechanically stripping an acrylic coated FBG had the same sensitivity as the acrylic coated FBG at the same center wavelength. Therefore it can be concluded that polyamide coated FBGs that were tested do have slightly higher strain sensitivity than acrylic and bare FBG.

\section{Strain Sensitivity Dependence on The Configuration.}

From Figure 4.5 it can be observed that serial gratings have a slightly higher sensitivity than collocated grating. The difference is $0.005 \mu \varepsilon^{-1}$ which is on the scale of the experimental error. The 1539 collocated grating had also a lower sensitivity than 1540 single grating. The difference is $0.007 \mu \varepsilon^{-1}$. Therefore it can be concluded that collocated gratings do have slightly lower strain sensitivity than single or serial gratings.

\subsubsection{Temperature Characterization Procedure and Results}

The temperature characterization has been done using a Peltier heater as shown in Figure 4.6. The test temperature range was from $70{ }^{\circ} \mathrm{C}$ down to $-10^{\circ} \mathrm{C}$, with the grating resting unstrained inside the heater. To minimize temperature losses the whole set-up was insulated with styrofoam. The tests started at room temperature, then the grating was heated to $70^{\circ} \mathrm{C}$ and then cooled to $-10^{\circ} \mathrm{C}$ in steps of $10^{\circ} \mathrm{C}$. The grating was allowed to temperature stabilize for 10 minutes at each temperature point and the transmission spectrum was collected using the Ando AQ6317B Optical Spectrum Analyzer. The resolution of the instrument was set to $0.05 \mathrm{~nm}$. The center wavelength of the peak was determined using the same procedure as described in section 4.2.1. The temperature sensitivity was determined from the slope of the linear fit of the wavelength response as 
shown in Figure 4.7. Wavelength normalized temperature characterization constants have been determined by dividing the obtained slopes of the data sets by the manufacturer specified grating center wavelength and are summarized in Table 4.3.

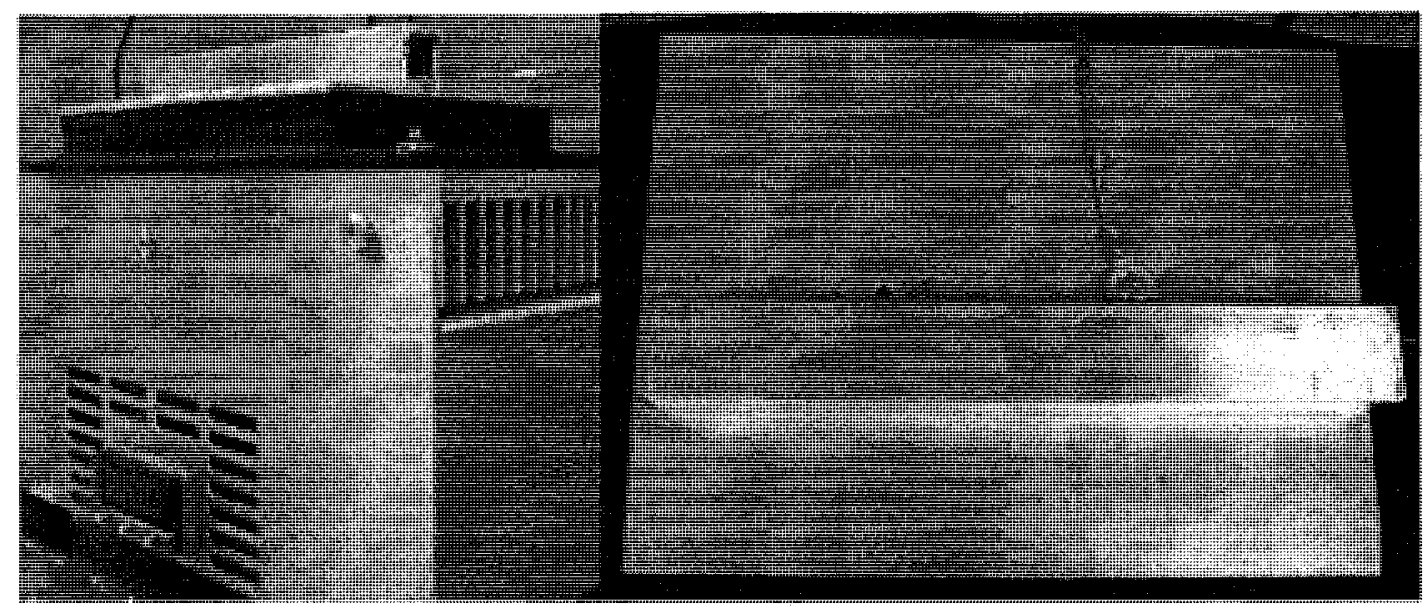

Figure 4.6: Uninsulated Peltier heater

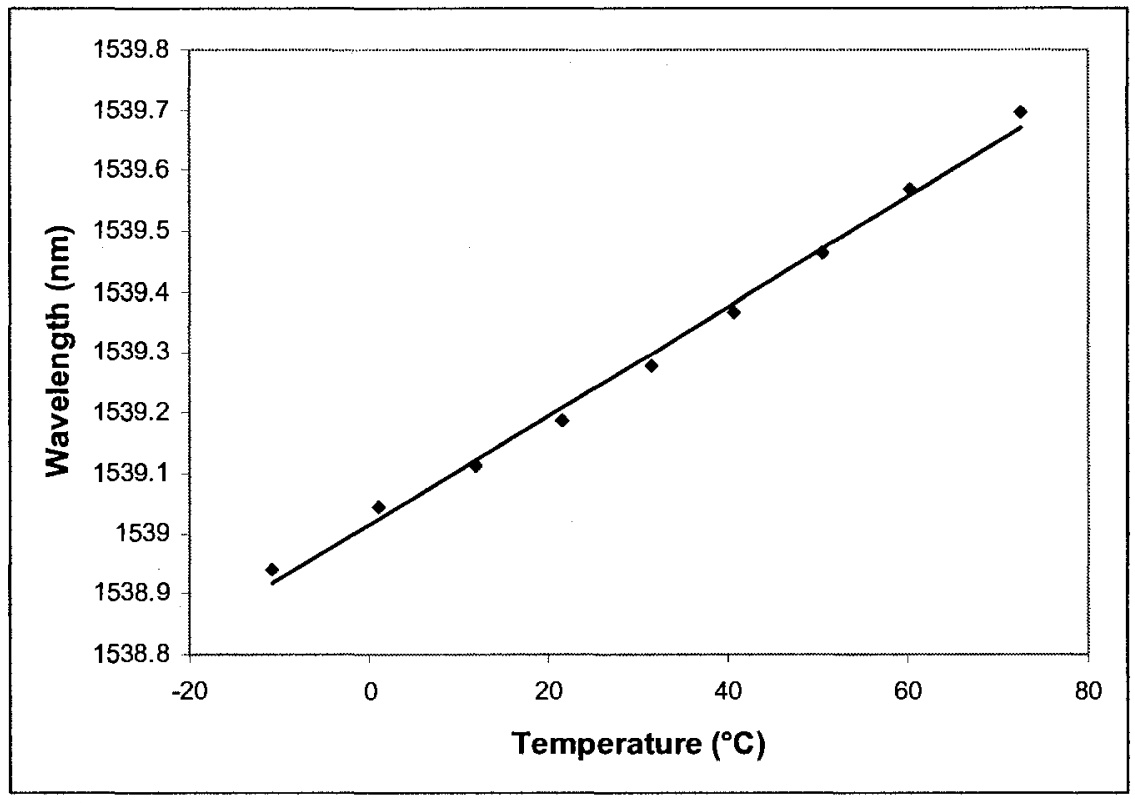

Figure 4.7: Typical temperature response. 


\begin{tabular}{|c|c|c|c|c|}
\hline Grating index & Coating & Wavelength (nm) & $K_{T} x 10^{6}{ }^{\circ} \mathrm{C}^{-1}$ & \pm Error $x 10^{-6}{ }^{\circ} \mathrm{C}^{-1}$ \\
\hline A-p1-02 & Polyamide & 1540 & 5.73 & 0.12 \\
\hline A-p1-03 & Polyamide & 1540 & 5.91 & 0.22 \\
\hline A-p2-08 & Polyamide & 1560 & 6.15 & 0.15 \\
\hline A-a1-12 & Acrylic & 1540 & 6.26 & 0.05 \\
\hline A-a1-12 & Acrylic & 1540 & 6.24 & 0.06 \\
\hline$A-a 2-10$ & Acrylic & 1560 & 6.30 & 0.05 \\
\hline$A-a 2-10$ & Acrylic & 1560 & 6.29 & 0.06 \\
\hline$A-a 2-10$ & Acrylic & 1560 & 6.30 & 0.06 \\
\hline A-a 1-12 & Bare & 1540 & 6.19 & 0.07 \\
\hline A-al-12 & Bare & 1540 & 6.29 & 0.07 \\
\hline \multirow{2}{*}{ D-II-11 } & \multirow{2}{*}{ Polyamide } & 1539 & 5.85 & 0.15 \\
\hline & & 1545 & 5.95 & 0.14 \\
\hline \multirow{2}{*}{ D-II-12 } & \multirow{2}{*}{ Polyamide } & 1539 & 5.84 & 0.10 \\
\hline & & 1545 & 5.96 & 0.14 \\
\hline \multirow{2}{*}{ D-II-13 } & \multirow{2}{*}{ Polyamide } & 1539 & 5.75 & 0.13 \\
\hline & & 1545 & 5.89 & 0.17 \\
\hline \multirow{2}{*}{ D-II-14 } & \multirow{2}{*}{ Polyamide } & 1539 & 5.85 & 0.17 \\
\hline & & 1545 & 5.85 & 0.18 \\
\hline \multirow{2}{*}{ D-II-14 } & \multirow{2}{*}{ Polyamide } & 1539 & 5.85 & 0.18 \\
\hline & & 1545 & 5.83 & 0.18 \\
\hline \multirow{2}{*}{ D-I-08 } & \multirow{2}{*}{ Polyamide } & 1539 & 5.87 & 0.18 \\
\hline & & 1545 & 5.84 & 0.21 \\
\hline \multirow{2}{*}{ D-I-09 } & \multirow{2}{*}{ Polyamide } & 1539 & 5.85 & 0.15 \\
\hline & & 1545 & 5.95 & 0.14 \\
\hline
\end{tabular}

Table 4.3: Temperature sensitivity results

As discussed in 3.2.2 the temperature sensitivity is expected to have a slightly nonlinear response. The polynomial fit gives an order of magnitude improvement over the linear fit in the $\chi^{2}$ value. However for applications not requiring better than a $2{ }^{\circ} \mathrm{C}$ accuracy the linear fit is usually a sufficient approximation Flockhart et al., 2004. 
Previously reported results of the temperature sensitivity of FBGs range from $6.13 \times 10^{-6}$ ${ }^{\circ} \mathrm{C}^{-1}$ to $8.2 \times 10^{-6}{ }^{\circ} \mathrm{C}^{-1}$ and are summarized in Table 4.4.

\begin{tabular}{lll}
\hline Reference & Wavelength $(\mathrm{nm})$ & $\boldsymbol{K}_{T}\left(\boldsymbol{x} 10^{-6}{ }^{\circ} \mathrm{C}^{-1}\right)$ \\
\hline [Sugden et al., 1997] & 1536 & 6.2 \\
[Flockhart et al., 2004] & 1524 & 8.1 \\
[Flockhart et al., 2004] & 1548 & 8.2 \\
[Flockhart et al., 2004] & 1572 & 8.15 \\
[Brady et al., 1996] & 1561 & 7.61 \\
[Caucheteur et al., 2004] & 1534 & 6.23 \\
[Caucheteur et al., 2004] & 1579 & 6.13 \\
\hline
\end{tabular}

Table 4.4: Previously reported temperature sensitivities

It should be noted that the temperature sensitivity is temperature dependent therefore the temperature range for which the sensitivity is quoted is particularly important. It has also been noted that doping concentration and hydrogenation do affect the temperature sensitivity. For example Sugden et al., 1997, has reported an increase in sensitivity for a heavily doped germanium fiber, $7.3 \times 10^{-6}{ }^{\circ} \mathrm{C}^{-1}$ and significantly decreased sensitivity for a Boron/Germanium doped fiber, $2.97 \times 10^{-6}{ }^{\circ} \mathrm{C}^{-1}$., has reported that the grating writing procedure also affects the temperature sensitivity. They experimented with the Boron/Germanium doped fibers and grating center wavelengths around $1550 \mathrm{~nm}$. The highest sensitivity that they observed was for a hydrogen free, long exposure gratings. It ranged from $7.1 \times 10^{-6}{ }^{\circ} \mathrm{C}^{-1}$ to $6.4 \times 10^{-6}{ }^{\circ} \mathrm{C}^{-1}$. Common commercially available gratings have a sensitivity of $6.1 \times 10^{-6}{ }^{\circ} \mathrm{C}^{-1}$ for hydrogen free fibers and $5.8 \times 10^{-6}{ }^{\circ} \mathrm{C}^{-1}$ for hydrogenated fibers. The lowest sensitivity was achieved for hydrogenated, long exposure gratings: $4.5 \times 10^{-6}{ }^{\circ} \mathrm{C}^{-1}$. Gratings in our study were short exposure gratings, 
written in standard telecommunication fibers with hydrogen loading. Therefore, from the previously reported data it is expected that their sensitivity would be around $6 \times 10^{-6}{ }^{\circ} \mathrm{C}^{-1}$. Experimentally determined temperature sensitivities ranged from $5.73 \times 10^{-6}{ }^{\circ} \mathrm{C}^{-1}$ to 6.30 $\mathrm{x} 10^{-6}{ }^{\circ} \mathrm{C}^{-1}$ the errors range from $0.05 \times 10^{-6}{ }^{\circ} \mathrm{C}^{-1}$ to $0.22 \times 10^{-6}{ }^{\circ} \mathrm{C}^{-1}$. The error is mainly attributed to the low number of data points taken and to the non-linearity of the response. Graphically, the experimentally determined sensitivity results are shown in Figure 4.8 and Figure 4.9.

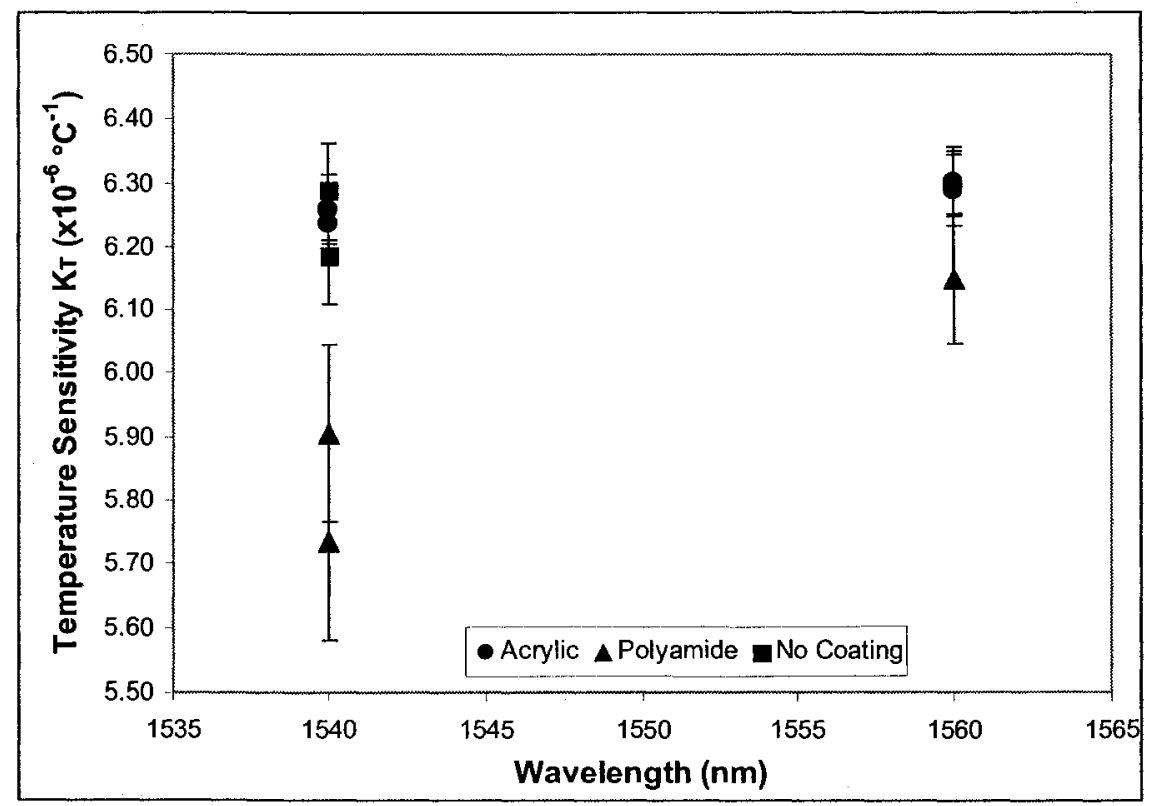

Figure 4.8: Temperature sensitivity for single gratings 


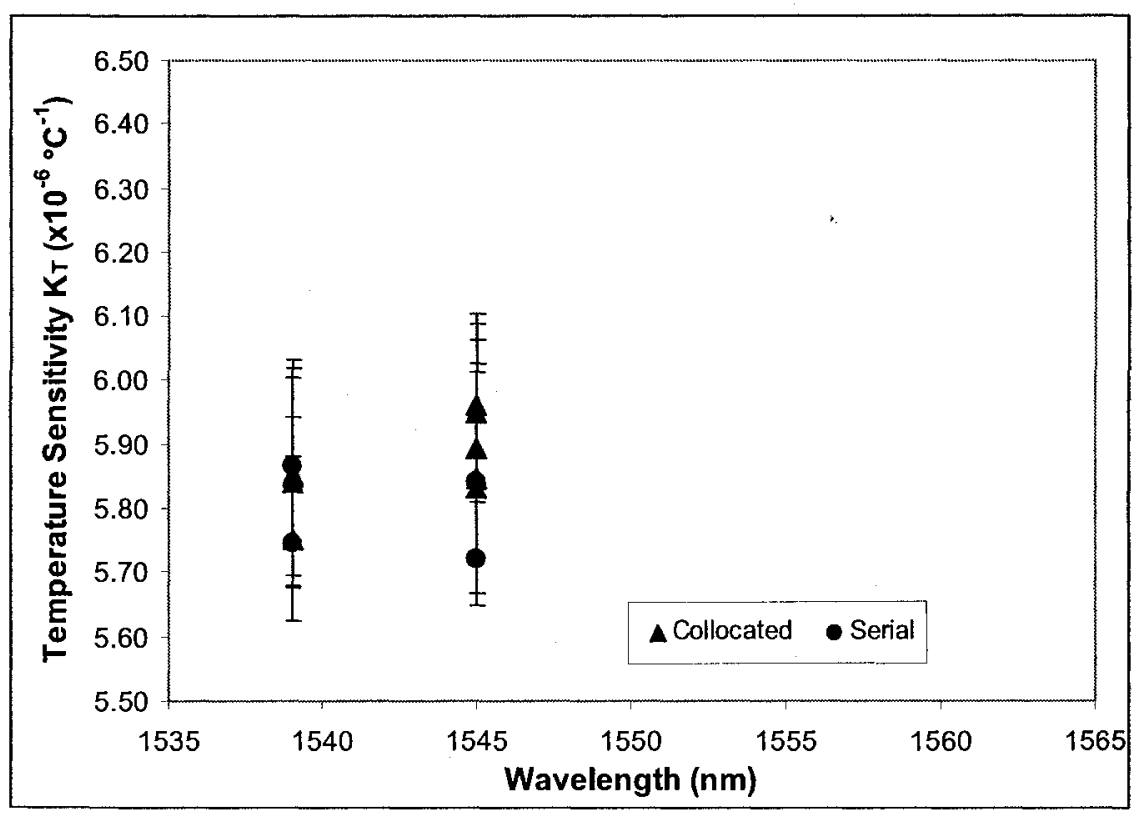

Figure 4.9: Temperature sensitivity for serial and collocated gratings

\section{Temperature sensitivity dependence on wavelength}

From Figure 4.8 a slight wavelength dependence of the temperature characterization coefficient can be observed. For polyamide coated gratings it is $\approx 0.4 \times 10^{-6}{ }^{\circ} \mathrm{C}^{-1} \mathrm{~nm}^{-1}$. For Acrylic coated gratings it is $\approx 0.06 \times 10^{-6}{ }^{\circ} \mathrm{C}^{-1} \mathrm{~nm}^{-1}$. Higher wavelengths are slightly more sensitive to temperature. The difference is on the scale of experimental error and is hard to evaluate exactly for a given wavelength range.

\section{Temperature Sensitivity Dependence on Coating}

From Figure 4.8 it can be also noticed that Acrylic coated fibers have higher temperature sensitivity than polyamide the variation is $\approx 0.5^{\circ} \mathrm{C}^{-1}$. It can be partially accounted for by the different temperature expansion coefficients of acrylate and polyamide. The Acrylate's thermal expansion coefficient is $68 \times 10^{-6} \mathrm{~m} / \mathrm{mK} \mathrm{EDL}, 2006$, 
which is two times bigger than the thermal expansion of polyamide $30 \times 10^{-6} \mathrm{~m} / \mathrm{mK}$, Engineers Handbook, 2006, and therefore it is expected that acrylic coated gratings would have a slightly higher sensitivity. A more plausible explanation is that as previously mentioned, acrylate coated gratings are made from SMF-28 fiber while the polyamide gratings are made from the OFS fiber which might have a slightly different composition and doping level which is known to affect the sensitivity. As a confirmation, stripped acrylic grating did not show any difference in sensitivity when compared to the acrylic coated gratings. Polyamide coated FBGs are slightly less sensitive to temperature than acrylic and bare FBGs however the difference is small for the -13 to $70{ }^{\circ} \mathrm{C}$ range.

\section{Temperture Sensitivity Dependence on Configuration}

As shown in Figure 4.9 there is no dependence of the temperature sensitivity on the FBG configuration.

\section{Wavelength Response For an Extended Temperature Range}

In the present work it has also been investigated whether gratings with different coatings behave differently at temperatures significantly below $0^{\circ} \mathrm{C}$. Figure 4.10 presents the experimental data for the 70 to $-40{ }^{\circ} \mathrm{C}$ cooling test for polyamide and acrylic coated FBGs. Both gratings exhibit some nonlinearity for temperatures below zero, however the one coated with polyamide retained roughly the same slope while the slope of the acrylic coated grating almost doubled. The increased sensitivity of the acrylic coated fiber is attributed to the low glass transition temperature of the acrylic recoat material Maier et al., 2004, Flockhart et al., 2004. The typical UV cured acrylic recoating material present 
on the fibre section containing the FBG (DSM Desolite: Desotech 950-200) has a glass transition temperature $\mathrm{Tg}$ around $-4{ }^{\circ} \mathrm{C}$ Desotech, 2007. The material properties change significantly over this temperature range, i.e. below $T \mathrm{~g}$ the coating is significantly harder Maier et al., 2004.

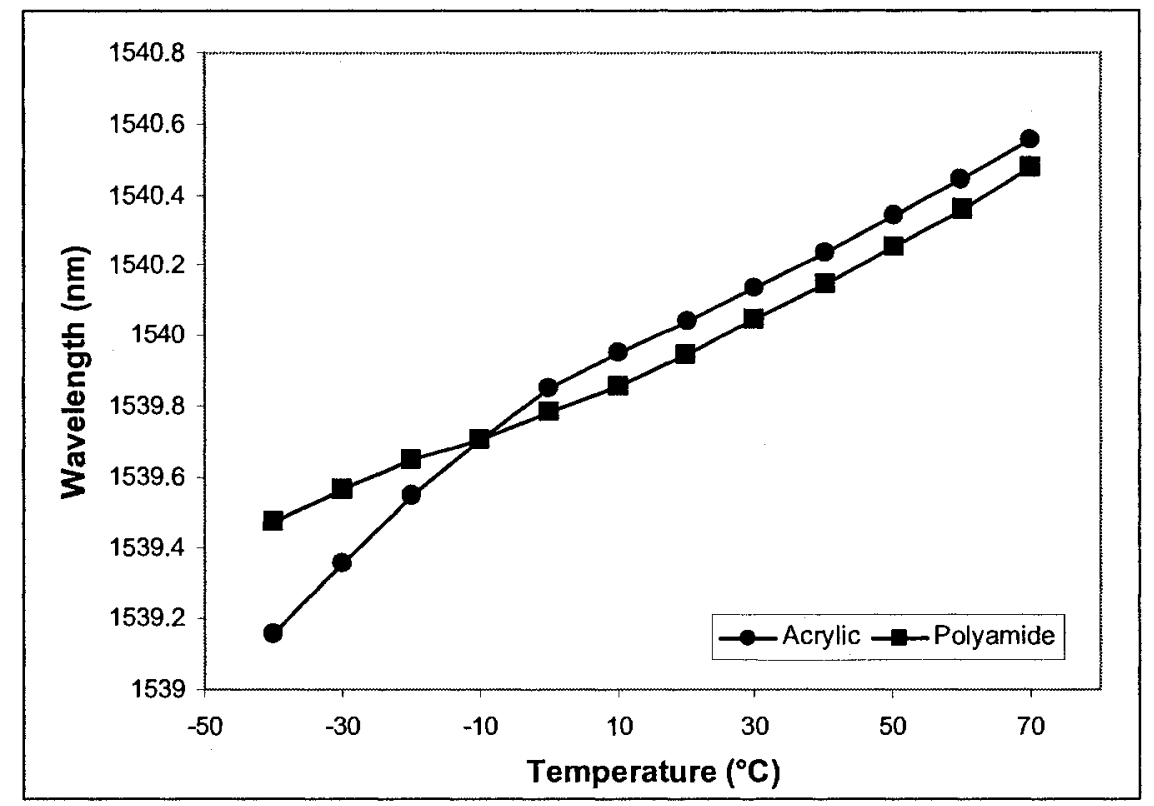

Figure 4.10: Temperature sensitivity of the Acrylic and Polyamide coated $1540 \mathrm{~nm} F B G$ for the expanded temperature range

\subsubsection{Combined Temperature and Strain Characterization}

This set of characterization tests were aimed at determining the temperature dependence of the strain response of the FBG. The temperature dependence of the strain sensitivity depends on the temperature dependence of the $\mathrm{P}_{\mathrm{e}}$ coefficient. To the best of our knowledge no suitable model exists, however the temperature dependence of the strain sensitivity has been studied experimentally. James et al., 2002 have reported that for gratings with center wavelengths of $1315 \mathrm{~nm}, 1310 \mathrm{~nm}$ and $1305 \mathrm{~nm}$ the strain response is constant for temperatures ranging from $20{ }^{\circ} \mathrm{C}$ down to $-268{ }^{\circ} \mathrm{C}$. However 
their strain characterization range was rather small - only 0 to $350 \mu \varepsilon$. Zhang et al., 2006 characterized gratings with center wavelengths ranging from $1540 \mathrm{~nm}$ to $1555 \mathrm{~nm}$ for a larger strain range: 0 to $5500 \mu \varepsilon$. Their conclusion was that for a temperature range of $-150^{\circ} \mathrm{C}$ to $42^{\circ} \mathrm{C}$ the strain sensitivity decreases linearly with a slope of $-5.19 \times 10^{-4} \varepsilon^{-10} \mathrm{C}^{-1}$.

Our experimental procedure was a combination of the temperature and strain characterization procedures. A bare optical fiber with room temperature center wavelength of $1540 \mathrm{~nm}$ was used for combined strain and temperature tests. The strain sensitivity was determined at $-12.4,20.4,20.6,45.2,71.5$ and $72{ }^{\circ} \mathrm{C}$. The set up was the same as for the strain characterization experiment with the addition of a Peltier heater to control the temperature (see Figure 4.11). The fiber containing the grating was routed through an aluminum tube and attached to a stationary post at one side and translation stage on the other. At every temperature point the grating was allowed to temperature stabilize and the strain characterization procedure was repeated in exactly the same way as discussed in the effective strain sensitivity measurement, section 4.2.1.

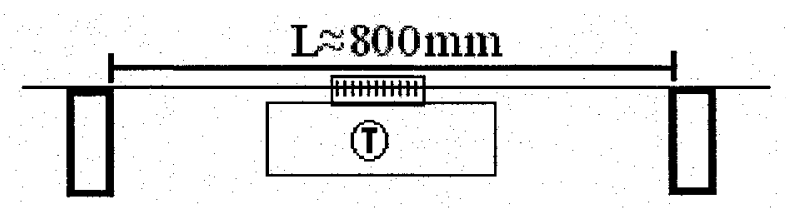

Figure 4.11: Combined temperature and strain experimental set-up

The results for the temperature dependence of the strain sensitivity are presented in Table 4.5: 


\begin{tabular}{ll}
\hline Temeperature $\left({ }^{\circ} \mathrm{C}\right)$ & $\mathrm{K}_{\varepsilon}\left(x 10^{-6}{ }^{\circ} \mathrm{C}^{1}\right)$ \\
\hline-12.4 & 0.800 \\
20.4 & 0.794 \\
20.6 & 0.798 \\
45.2 & 0.792 \\
71.5 & 0.792 \\
72 & 0.791
\end{tabular}

Table 4.5: Temperature dependence of the strain sensitivity for strain range of $2500 \mu \varepsilon$.

Graphically the results are shown in Figure 4.12 It can be observed that strain sensitivity decreases for increasing temperatures with the slope of $-0.1 \varepsilon^{-1}{ }^{\circ} \mathrm{C}^{-1}$. The maximum variation between the $72{ }^{\circ} \mathrm{C}$ and $-12.4{ }^{\circ} \mathrm{C}$ measurement is $1.08 \%$. The increase in sensitivity with decreasing temperature is slightly less than $-0.519 \varepsilon^{-1}{ }^{\circ} \mathrm{C}^{-1}$ reported by Zhang et al., 2006. The discrepancy can be attributed to the limited accuracy of our experimental set-up. Our results are based on only six points for a smaller temperature and strain range. 


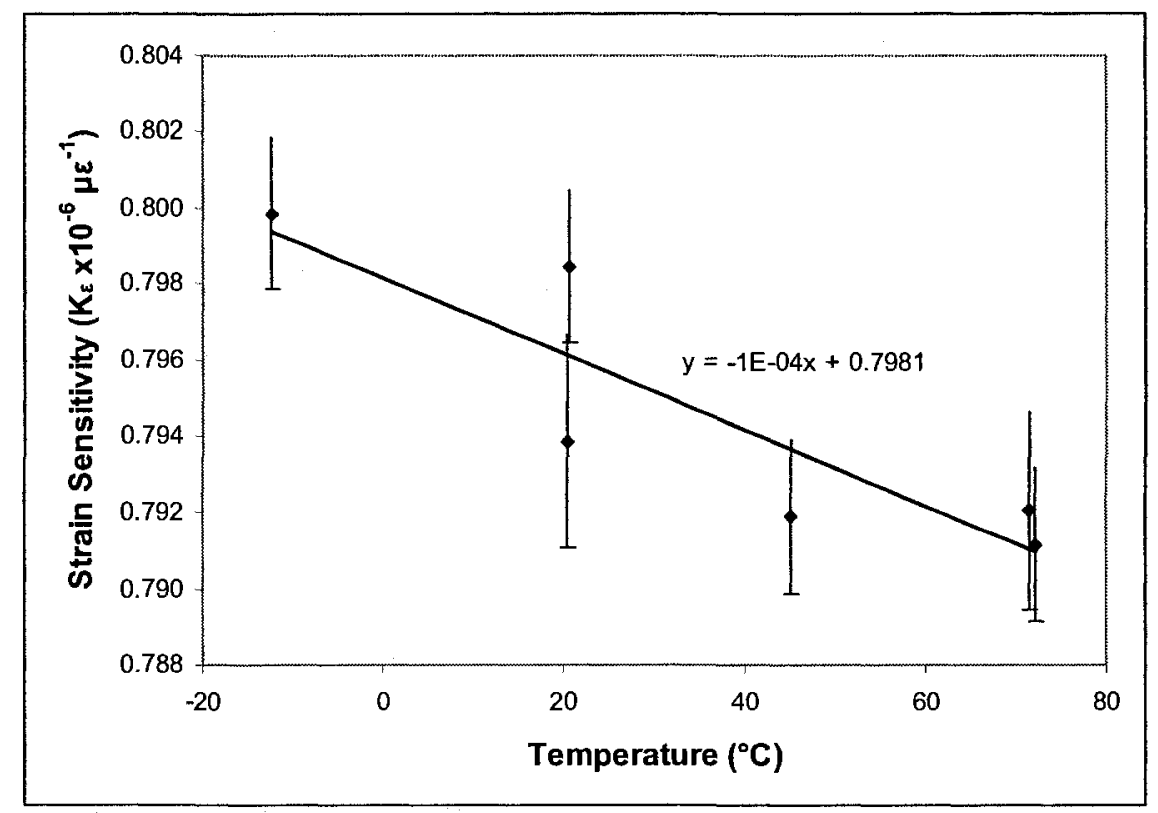

Figure 4.12: Temperature dependence of the strain sensitivity

Based on our results and the ones previously reported by Zhang et al., 2006, for temperatures ranging -12 to $72{ }^{\circ} \mathrm{C}$ the strain sensitivity of the gratings within the $\mathrm{C}$-band is expected to vary by $1 \%$ with higher sensitivity for the lower temperatures and lower sensitivity for higher temperatures.

\subsubsection{Dual Grating Error Analysis}

To analyze the feasibility of using a dual grating sensor system for temperature and strain discrimination, the condition number of the sensitivity matrix discussed in section 3.2.4 must be found. Using the Matlab cond( ) function the condition number of the $1539 / 1545$ grating pairs tested in this study have been calculated and are shown in Table 4.6. 


\begin{tabular}{lllll}
\hline Grating & Wavelength $(\mathrm{nm})$ & $\boldsymbol{K}_{T}\left(\mathbf{1 0}^{-6}{ }^{\circ} \mathrm{C}^{-1}\right)$ & $\boldsymbol{K}_{\varepsilon}\left(10^{-6} \mu \varepsilon^{-1}\right)$ & Condition number \\
\hline \multirow{2}{*}{ D-I-pa-08 } & 1539 & 5.87 & 0.798 & 2413 \\
& 1545 & 5.84 & 0.800 & \\
\hline \multirow{2}{*}{ D-II-pc-14 } & 1539 & 5.85 & 0.794 & 2749 \\
& 1545 & 5.83 & 0.795 & \\
\hline
\end{tabular}

Table 4.6: Dual grating matrix condition numbers

The condition numbers are extremely high, which implies that these grating pairs would not allow the discrimination between strain and temperature. The reason for such high condition numbers is their extremely similar temperature and strain sensitivity coefficients for the two wavelengths. To decrease the condition number either the wavelength separation between the two gratings should be increased or the physical sensitivity of one of the gratings significantly altered.

To confirm the theoretical hypothesis the, D-II-pc-14, 1539/1545nm dual, collocated, polyamide coated grating was subjected to temperature changes ranging from -29 to $+40^{\circ} \mathrm{C}$ from the reference temperature of $20^{\circ} \mathrm{C}$ and strains up to $2800 \mu \varepsilon$. The experimental procedure was the same as the one described in section 4.2.3. Applied and calculated temperature and strain are shown in Table 4.7. From the results shown in Table 4.7. The maximum strain error was $2300 \mu \varepsilon$ and the maximum temperature error was $310^{\circ} \mathrm{C}$. This confirms that indeed a $1539 / 1545 \mathrm{~nm}$ collocated grating is not suitable for temperature strain discrimination. 


\begin{tabular}{|c|c|c|c|c|}
\hline & & \multicolumn{3}{|c|}{ Applied temperature } \\
\hline & & $-29^{\circ} \mathrm{C}$ & $1^{\circ} \mathrm{C}$ & $40^{\circ} \mathrm{C}$ \\
\hline \multirow{6}{*}{$\begin{array}{l}\text { Applied } \\
\text { strain }\end{array}$} & $2791 \mu \varepsilon$ & $\begin{array}{c}-66^{\circ} \mathrm{C} \\
3085 \mu \varepsilon\end{array}$ & $\begin{array}{c}142^{\circ} \mathrm{C} \\
1750 \mu \varepsilon\end{array}$ & $\begin{array}{c}-4^{\circ} \mathrm{C} \\
3125 \mu \varepsilon\end{array}$ \\
\hline & $2114 \mu \varepsilon$ & $\begin{array}{l}\text { NA } \\
\text { NA }\end{array}$ & $\begin{array}{l}-123^{\circ} \mathrm{C} \\
3025 \mu \varepsilon\end{array}$ & $\begin{array}{l}\text { NA } \\
\text { NA }\end{array}$ \\
\hline & $1436 \mu \varepsilon$ & $\begin{array}{c}37^{\circ} \mathrm{C} \\
965 \mu \varepsilon \\
\end{array}$ & $\begin{array}{c}-7^{\circ} \mathrm{C} \\
1485 \mu \varepsilon\end{array}$ & $\begin{array}{l}-100^{\circ} \mathrm{C} \\
2468 \mu \varepsilon \\
\end{array}$ \\
\hline & $759 \mu \varepsilon$ & $\begin{array}{l}\mathrm{NA} \\
\mathrm{NA}\end{array}$ & $\begin{array}{c}311^{\circ} \mathrm{C} \\
-1543 \mu \varepsilon \\
\end{array}$ & $\begin{array}{l}\text { NA } \\
\text { NA }\end{array}$ \\
\hline & $81 \mu \varepsilon$ & $\begin{array}{l}\text { NA } \\
\text { NA }\end{array}$ & $\begin{array}{l}136^{\circ} \mathrm{C} \\
-909 \mu \varepsilon\end{array}$ & $\begin{array}{l}41^{\circ} \mathrm{C} \\
85 \mu \varepsilon\end{array}$ \\
\hline & $0 \mu \varepsilon$ & $\begin{array}{c}33^{\circ} \mathrm{C} \\
-438 \mu \varepsilon \\
\end{array}$ & $\begin{array}{l}\text { NA } \\
\text { NA }\end{array}$ & $\begin{array}{l}\text { NA } \\
\text { NA }\end{array}$ \\
\hline
\end{tabular}

Table 4.7: Applied and calculated temperature and strain.

\subsection{Bonded Temperature and Strain Sensitivity Characterization.}

In the previous section the sensitivity of free gratings has been experimentally determined. In this section the same gratings are epoxy bonded to aluminium and the temperature and strain characterization is repeated. As in the previous section the results are then grouped and the variations in sensitivity due to wavelength, coating and configuration are analyzed and compared to the sensitivities obtained for the free FBGs.

\subsubsection{Bonding Procedure}

Two identical T2024 aluminium plates labelled FOS1 and FOS2 with dimension $482.6 \times 50.8 \times 6.35 \mathrm{~mm}(19 \times 2 \times 0.25 \mathrm{in})$ were selected as the substrates for the bonded tests. 5 gratings were bonded on FOS1 and 4 gratings were bonded on FOS2. The gratings were positioned in the center of a beam one besides another with the separation of $2 \mathrm{~mm}$ as shown in Figure 4.13. That way the effects of non-uniform strains at the edges are 
minimized and it can be assumed that the gratings only see uniform axial and compressive transverse strain when the beams are elongated.
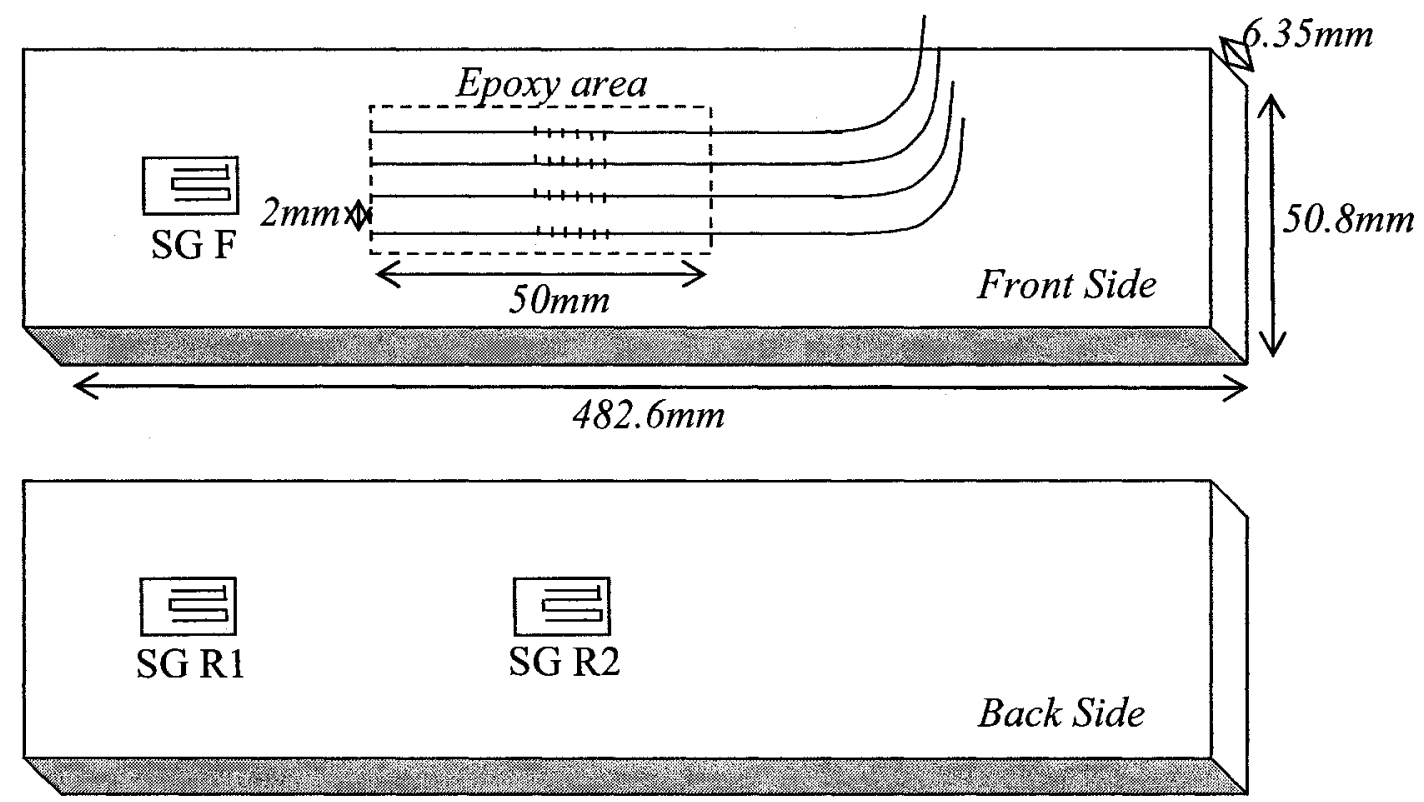

Figure 4.13: Grating layout

To assure that the gratings were in as close contact with the substrate as possible they were first taped down at the edges to the substrate with the masking tape and then an even, bubble free, $\approx 1 \mathrm{~mm}$ thick layer of Vishay $\mathrm{M}$ bond AE-10 epoxy was poured on top of the gratings. The effective bonded gauge length was $50 \mathrm{~mm}$. The epoxy was then allowed to cure for 24 hours at room temperature.

\subsubsection{Resistive Strain Gauges}

To measure strains in the beams Micro-Measurements EA-06-031CE-350 $350 \Omega$ resistive strain gauges were attached as shown in Figure 4.13. As it was impossible to measure strain in the same spot where the gratings were mounted three strain gauges 
were used to ensure the strain is evenly distributed across the substrate. SG F was attached on the same side as the FBGs, SG R1 was on the back side across from the SG F and SG R2 was on the back side across from the FBGs. SG R2 readings were considered the true strain and SG R1 and SG F were used to verify that the strain is uniform across the gauge length, on both sides of the beam.

Over the course of strain testing a number of resistive strain gauge issues have been identified. Figure 4.14 shows the strain data read by three strain gauges vs. load for FOS2 during the first trial of testing.

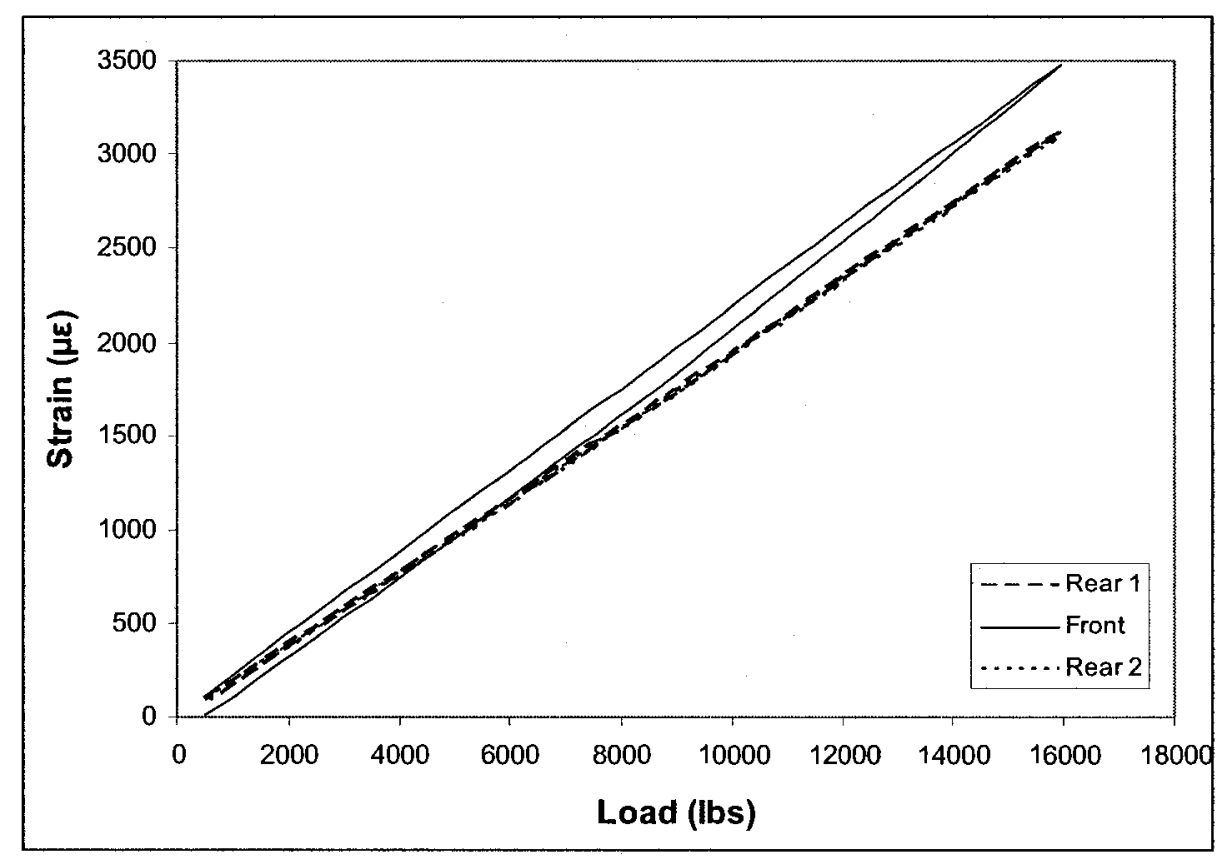

Figure 4.14: Resistive strain gauge data FOS2

It can be observed that front gauge exhibits a noticeable amount of hysterisis and is deviating from the two back gauges. Figure 4.15 shows the same data after the front gauge has been replaced, now all three gauges are reading the same strain. 


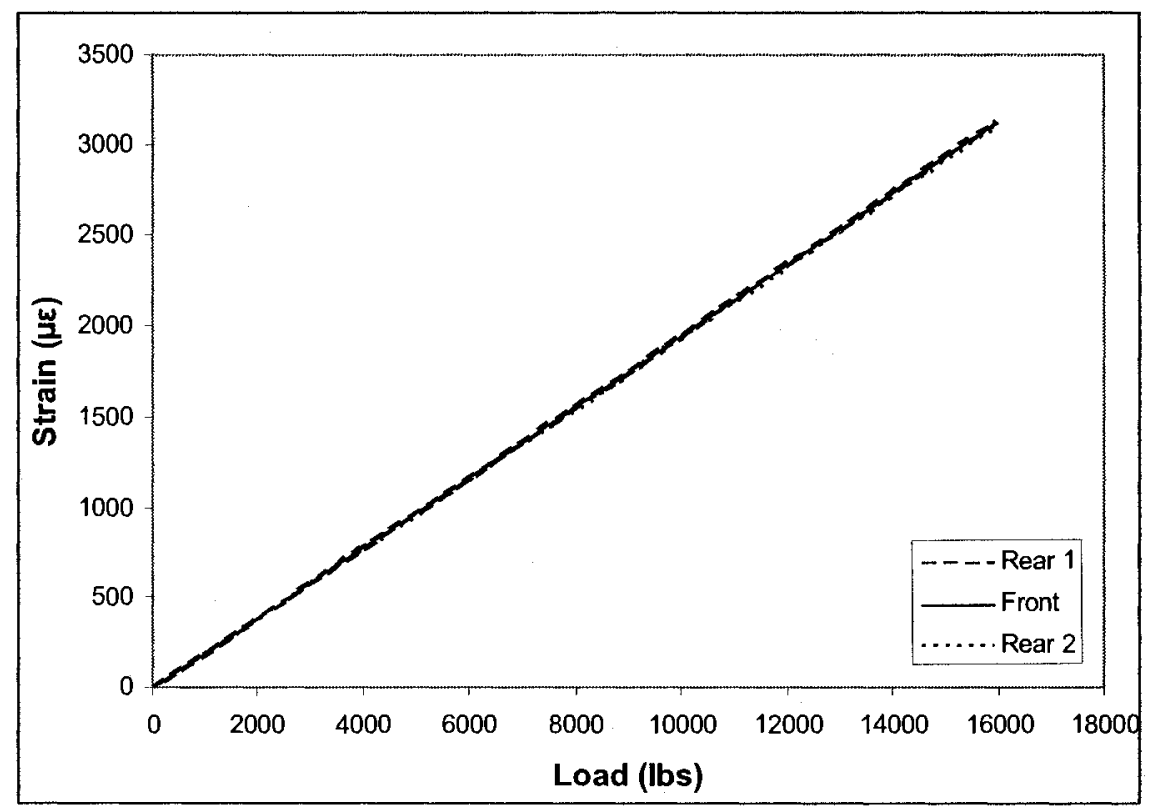

Figure 4.15: Resistive strain gauge FOS2 after the front gauge has been replaced

A similar issue has been observed on FOS1, again as can be seen from Figure 4.16 the front gauge is slightly deviating from the two back gauges.

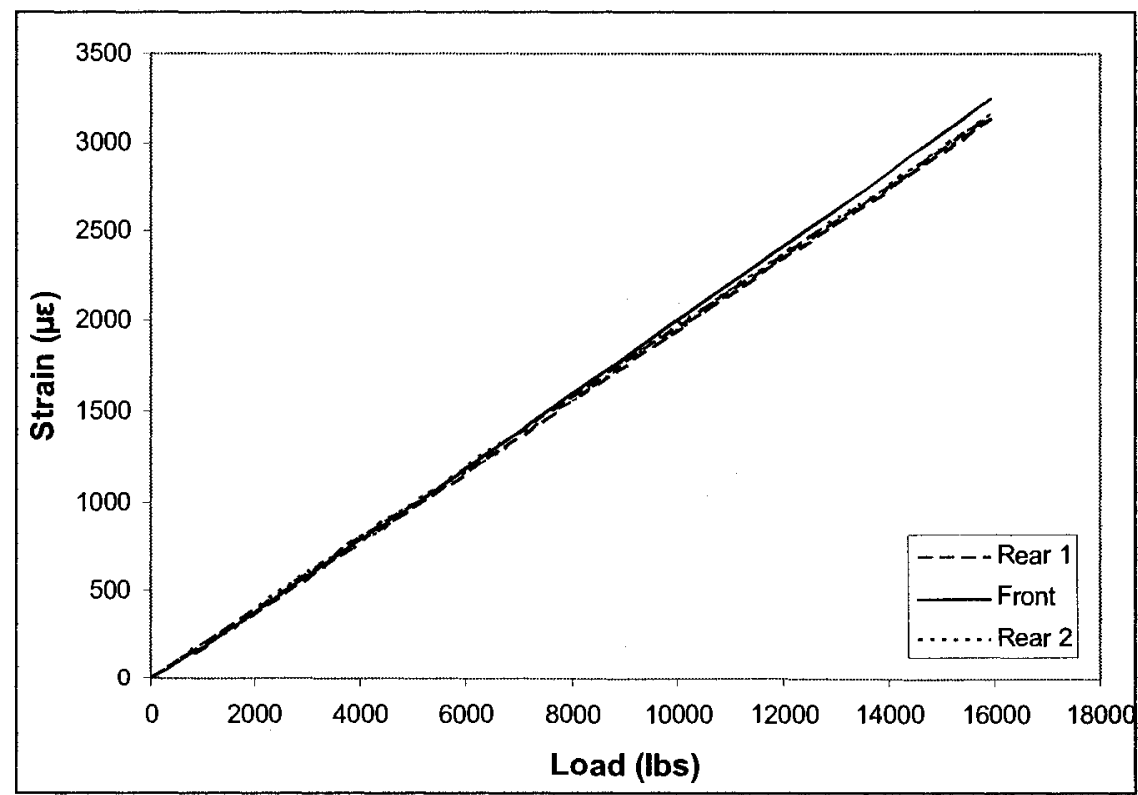

Figure 4.16: Resistive strain gauge FOS1 
Figure 4.17 shows the response with the front gauge replaced, as in the previous case the readings are now more consistent. The initial deviations are attributed to mounting errors which led to the poor contact of the strain gauges with a substrate.

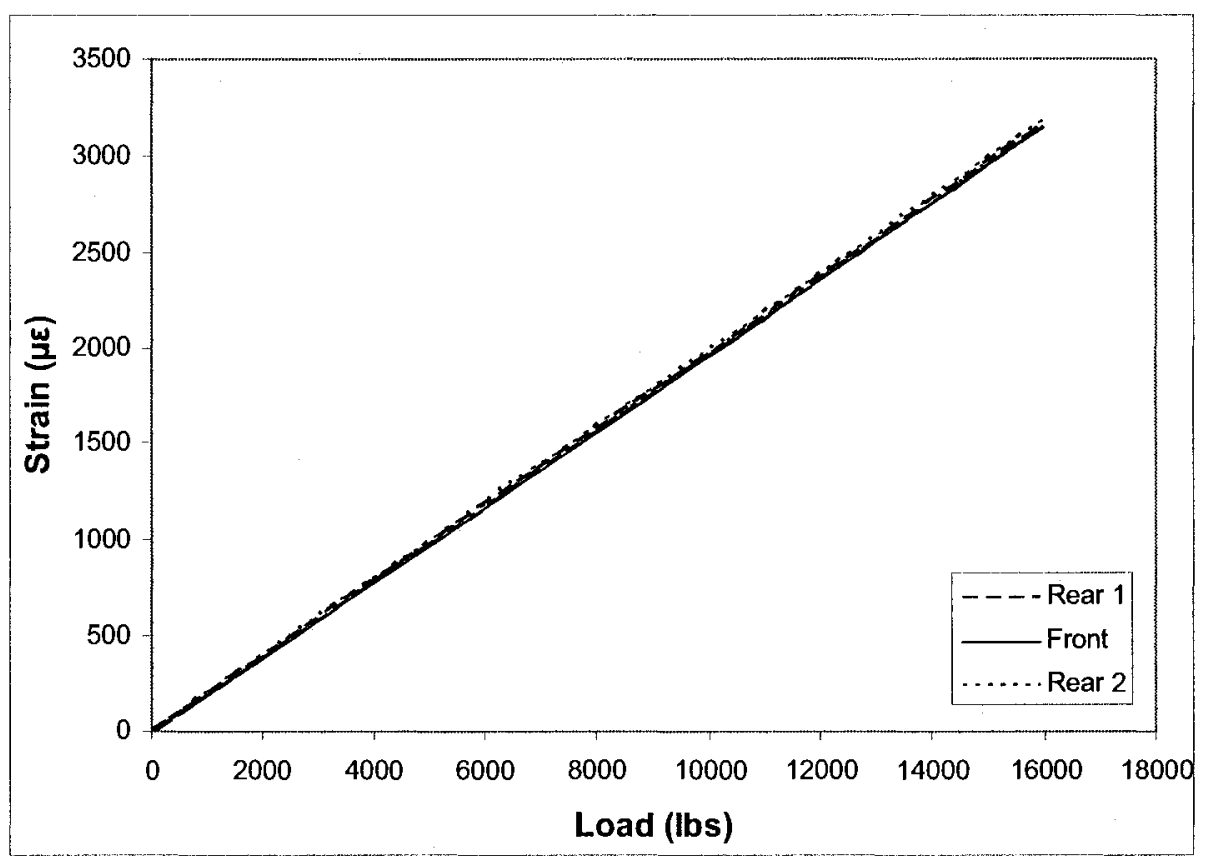

Figure 4.17: Resistive strain gauge FOS1

\subsubsection{Bonded Strain Characterization Procedure and Results}

The strain characterization was carried out on MTS tensile test machines. The load was applied in 20 steps of $800 \mathrm{lbs}(3.6 \mathrm{kN})$ up to a maximum load of $16000 \mathrm{lbs}(71.2 \mathrm{kN})$ and then back down to 0lbs. At each step the load was held constant for 40 seconds. The strain gauge and load data were continuously collected by data acquisition board with the sampling frequency of $1 \mathrm{~Hz}$. The wavelength data was collected using the HP86120C wavemeter with the sampling frequency of $0.5 \mathrm{~Hz}$. At each step, where the load was constant, strain and wavelength data were averaged and then strain was plotted versus wavelength. The slope of the graph was then used to calculate the wavelength normalized 
strain sensitivity of a grating under test. Both the up and down cycles were used in the calculation of the slope as little hysterisis was observed.

A number of steps have been taken to make sure that there was no epoxy degradation and that the optical gauges are producing repeatable results. Initially FOS2 has been cycled 6 times up and down with the wavelength data recorded continuously on a polyamide coated dual collocated FBG. The corresponding characterisation plots for the $1539 \mathrm{~nm}$ grating are shown in Figure 4.18.

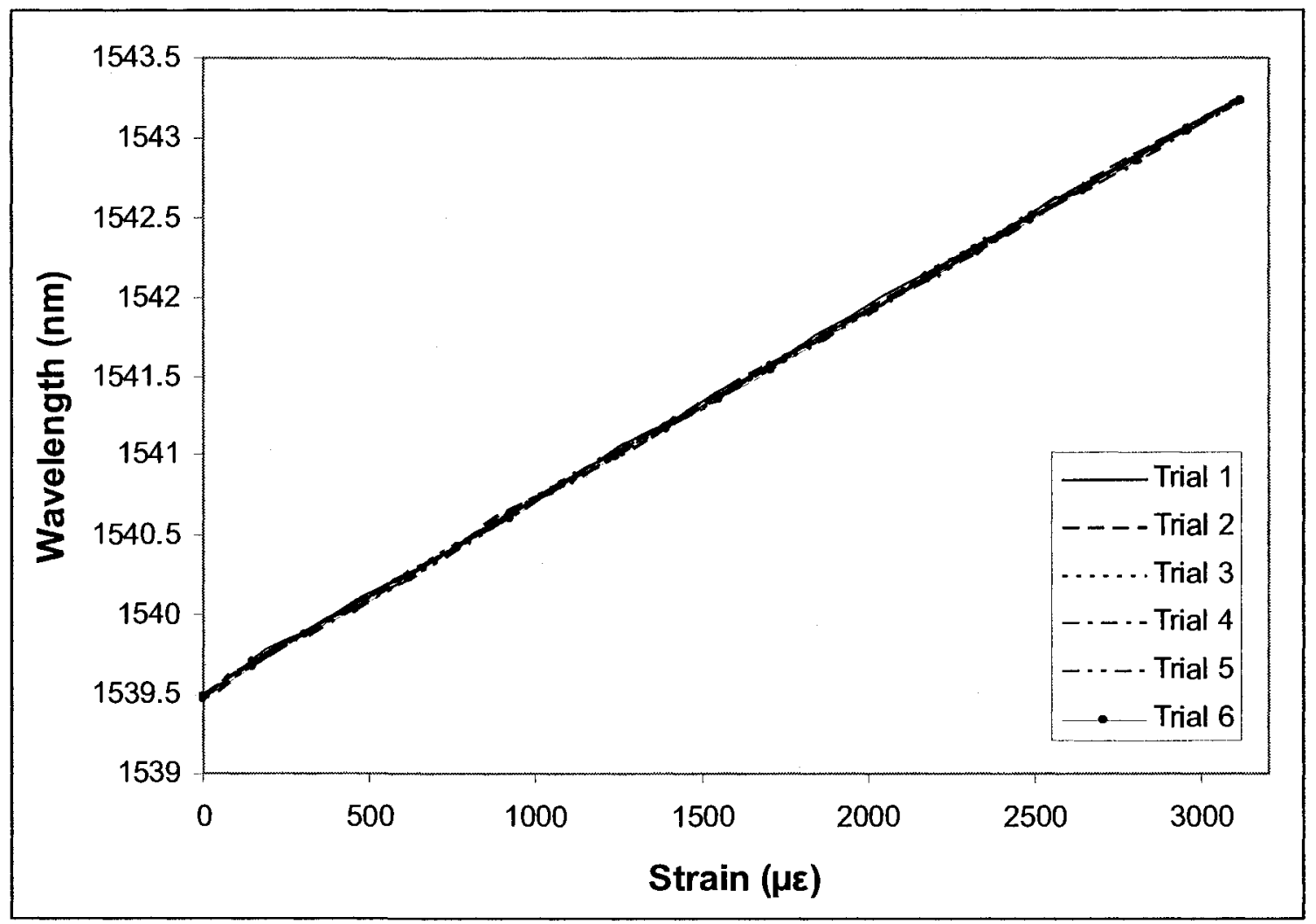

Figure 4.18: Repeatability results for polyamide coated dual FBG on FOS2

As can be seen from Figure 4.18 all 6 trials produced the same result without any deviations. That is despite the fact that in trials $1-4$ only two strain gauges were used as a 
reference while for trials 5 and 6 the third strain gauge was replaced (for the reasons explained in section 4.3.2) and all three strain gauges were used in the analysis. The sensitivity slopes were calculated for all six trials and the standard deviation did not exceed $0.003 \mu \varepsilon^{-1}$. This translates into a maximum error of $0.4 \%$ on the experimentally obtained strain sensitivity. The rest of the gratings on FOS2 were tested only once and the error reported is the error of the slope.

FOS1 was tested using the same procedure and the initial epoxy disband test was repeated 3 times on an acrylate coated $1560 \mathrm{~nm}$ single FBG. The corresponding characterization plots are shown in Figure 4.19.

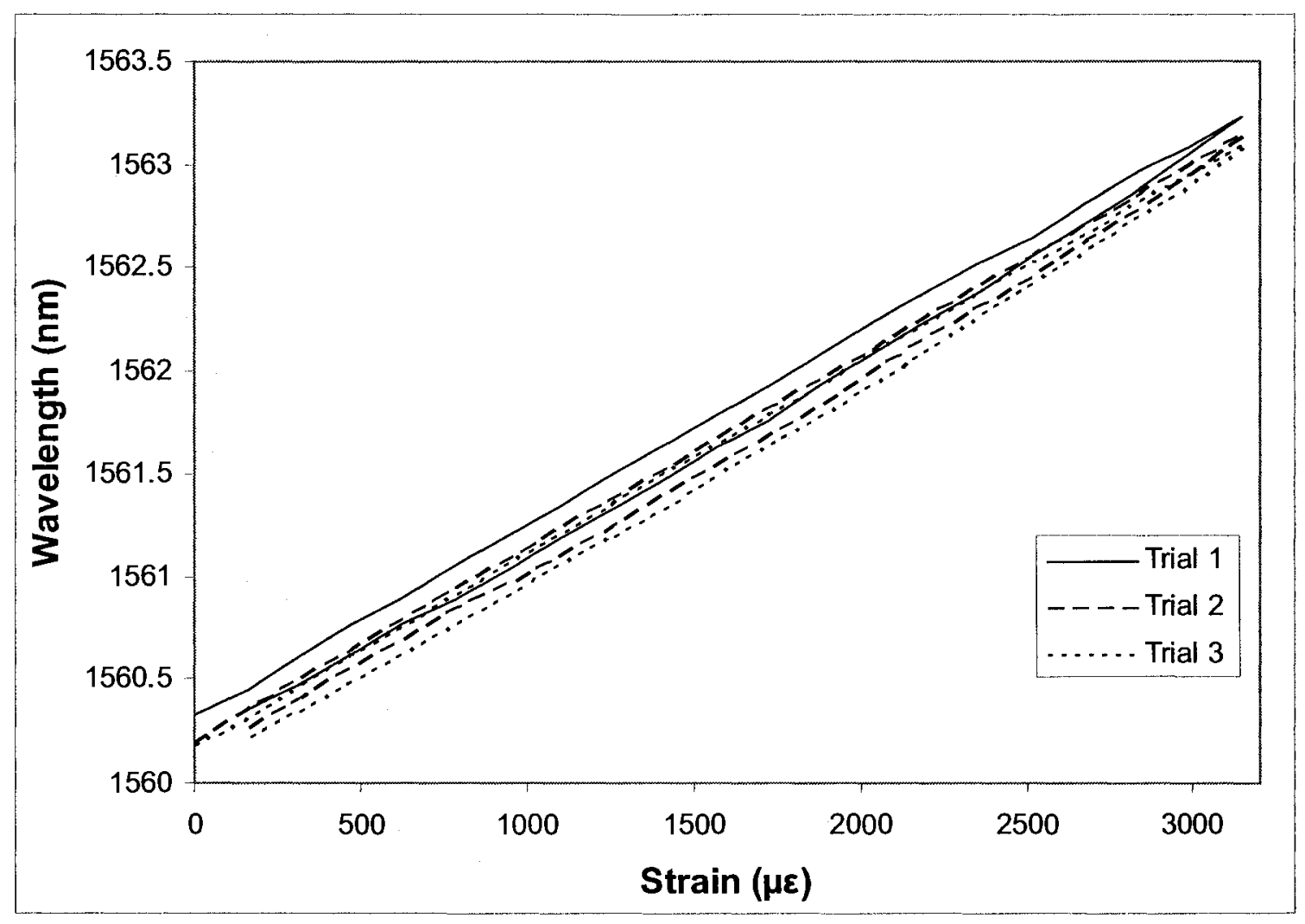

Figure 4.19: Repeatability results for acrylate coated single FBG on FOSI 
This time some hysterisis and overall center wavelength decrease can be observed from trial 1 to trial 3. Such response is expected because the acrylate is a thick and soft coating and does not transfer strain very well. This issue will be discussed further later. Despite the apparent issues the slopes were very similar and the three standard deviations were still $0.005 \times 10^{-6} \mu \varepsilon^{-1}$, which translates to a $0.9 \%$ error on the obtained sensitivity.

Obtained strain sensitivity for all of the bonded gratings can be found in Table 4.8. The last $\%$ difference column represents the discrepancy with the free characterization results. Graphically the results are shown in Figure 4.20 for single gratings and Figure 4.21 for dual gratings.

\begin{tabular}{|c|c|c|c|c|c|c|}
\hline $\begin{array}{l}\text { Grating } \\
\text { index }\end{array}$ & Coating & Plate & $\begin{array}{l}\text { Wavelength } \\
(\mathrm{nm})\end{array}$ & $\begin{array}{l}K \varepsilon \\
10^{-6} \mu \varepsilon^{-1} \\
\end{array}$ & $\begin{array}{l} \pm \text { Error } \\
10^{-6} \mu \varepsilon^{-1}\end{array}$ & $\begin{array}{l}\% \\
\text { difference }\end{array}$ \\
\hline A-p1-02 & Polyamide & FOS 2 & 1540 & 0.778 & 0.002 & -2.9 \\
\hline A-p1-03 & Polyamide & FOS 1 & 1540 & 0.771 & 0.002 & -3.6 \\
\hline A-p2-09 & Polyamide & FOS 1 & 1560 & 0.764 & 0.002 & -4.2 \\
\hline A-a1-13 & Acrylic & FOS 1 & 1540 & 0.545 & 0.003 & -31.4 \\
\hline$A-a 2-10$ & Acrylic & FOS 1 & 1560 & 0.610 & 0.002 & -23.0 \\
\hline A-a1-12 & No Coating & FOS 1 & 1540 & 0.752 & 0.004 & -5.2 \\
\hline \multirow{2}{*}{ D-II-pc-14 } & \multirow{2}{*}{ Polyamide } & \multirow{2}{*}{ FOS 2} & 1539 & 0.779 & 0.001 & -1.9 \\
\hline & & & 1545 & 0.773 & 0.003 & -2.8 \\
\hline \multirow{2}{*}{ D-II-pc-12 } & \multirow{2}{*}{ Polyamide } & \multirow{2}{*}{ FOS 2} & 1539 & 0.780 & 0.001 & NA \\
\hline & & & 1545 & 0.775 & 0.002 & NA \\
\hline \multirow{2}{*}{ D-I-pa-08 } & \multirow{2}{*}{ Polyamide } & \multirow{2}{*}{ FOS 2} & 1539 & 0.788 & 0.002 & -1.4 \\
\hline & & & 1545 & 0.776 & 0.002 & -3.0 \\
\hline
\end{tabular}

Table 4.8: Bonded strain sensitivity results 


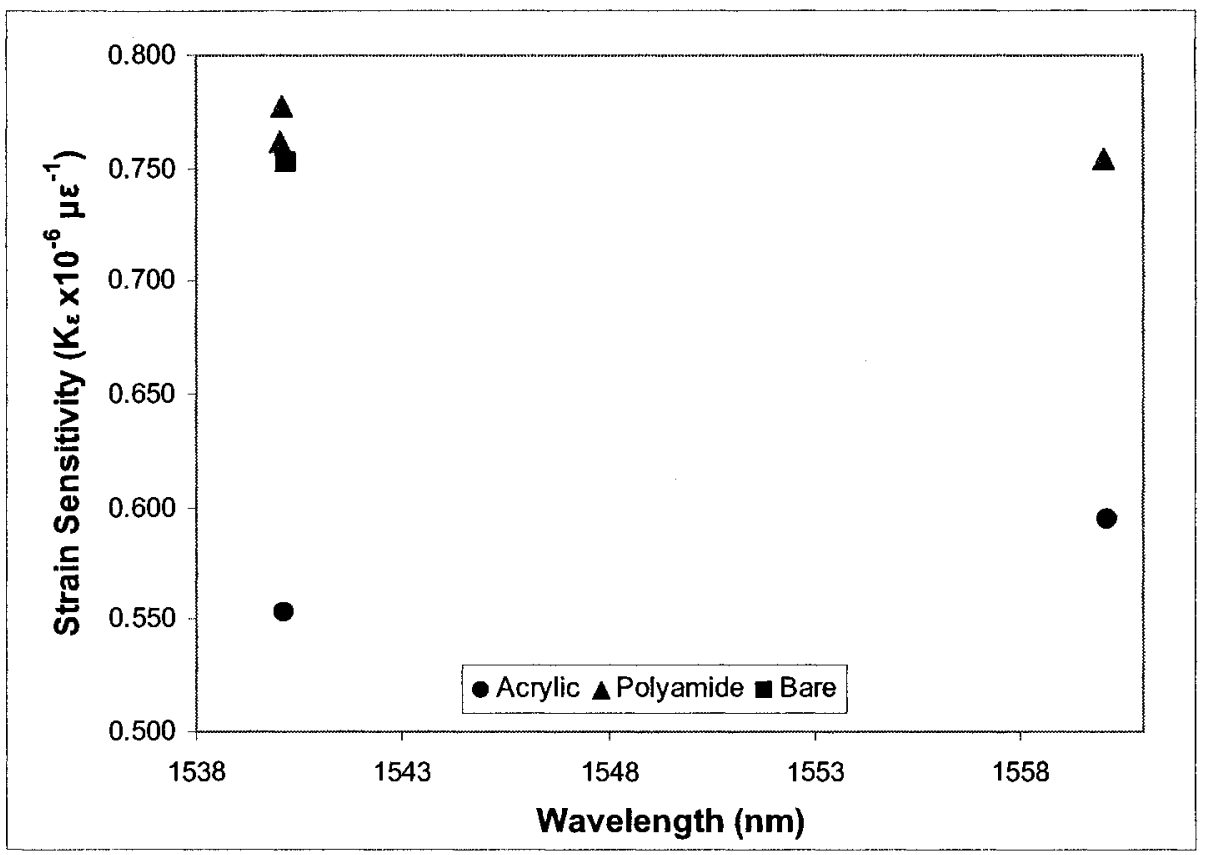

Figure 4.20: Strain sensitivity single gratings

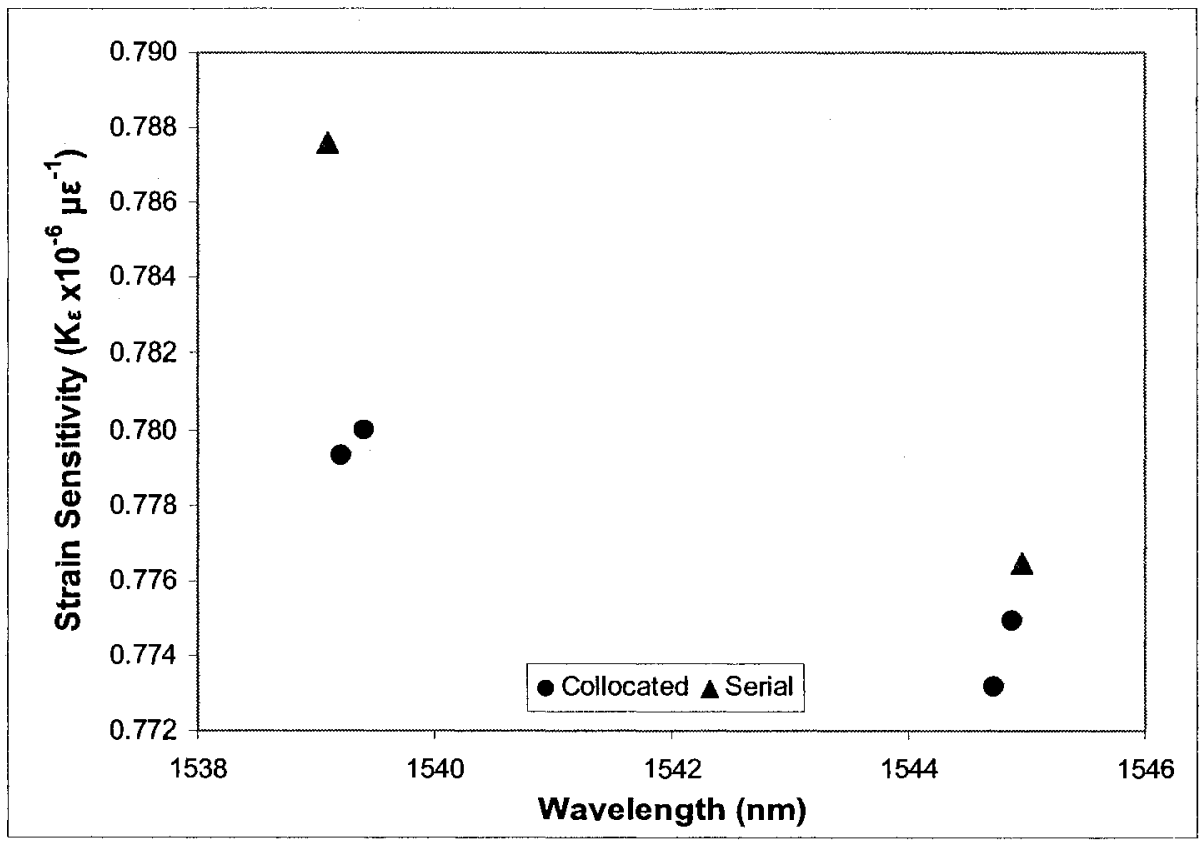

Figure 4.21: Strain sensitivity dual gratings 


\section{Strain Sensitivity Dependence on Wavelength:}

As can be seen from Figure 4.20Figure 4.21 the strain sensitivity did not depend significantly on the wavelength. The only exception is abnormally low sensitivity for the higher wavelength gratings in the dual grating pairs, especially for the serial grating. This is contrary to what has been observed for free gratings and is most likely due to the different FBG interrogators used in the two experiments. Free gratings have been interrogated with a spectrum analyzer and the peak data has been extracted manually, while bonded gratings have been interrogated with a wavemeter which automatically outputs the peak data. To verify the validity of the wave meter all gratings on FOS 2 have been retested with a Micron optics si 720 sensing analyzer. The reflection spectrum of the polyamide coated serial dual grating with the center wavelengths of 1539 and $1545 \mathrm{~nm}$ is shown in Figure 4.22. It can be seen that the peaks are actually somewhat noisy and indeed the wavemeter might have had problems resolving the true center wavelength. Figure 4.23 shows the spectrum shifted such that the $1539 \mathrm{~nm}$ peaks are superimposed. It shows the differential shift due to strain for the two gratings. It can be seen that the 1545 $\mathrm{nm}$ grating shifted slightly more then the $1539 \mathrm{~nm}$ grating, which is consistent with the previous findings. The comparison of the wavemeter and spectrum analyzer results is presented in Table 4.9 , the percent difference is less then $2 \%$. 


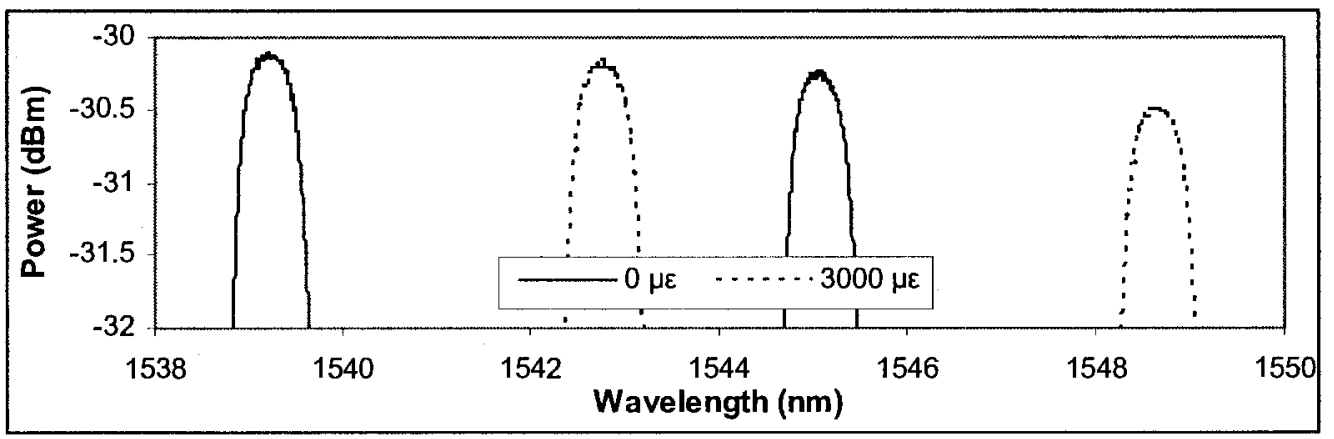

Figure 4.22: Reflection spectrum for two different loads, measured with si720

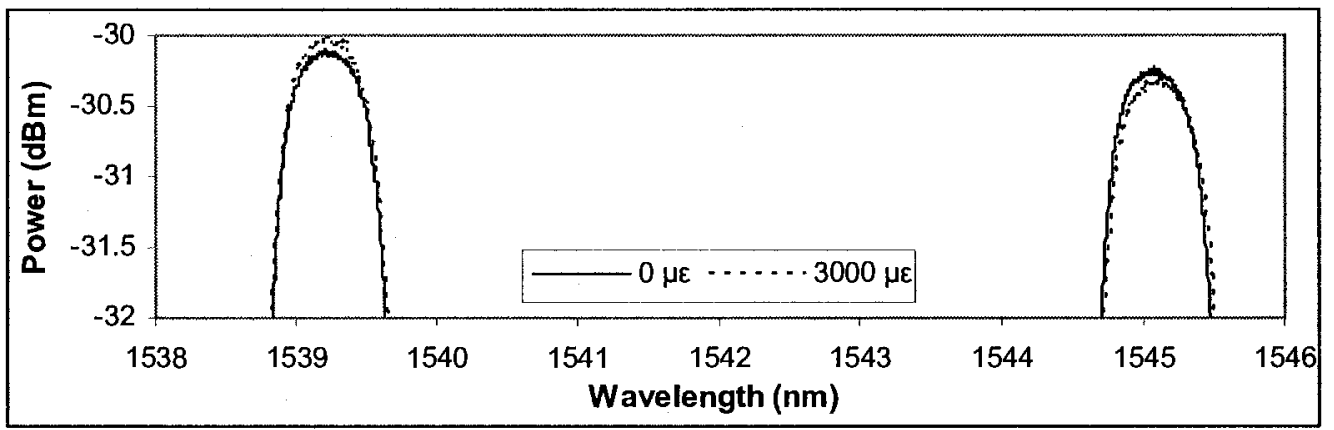

Figure 4.23: Superimposed reflection spectrum for two different loads, measured with si720

\begin{tabular}{|c|c|c|c|c|c|c|}
\hline $\begin{array}{l}\text { Grating } \\
\text { Index }\end{array}$ & Coating & Plate & $\begin{array}{l}\text { Wavelength } \\
\text { (nm) }\end{array}$ & $\begin{array}{l}\text { KE wavemeter } \\
10^{-6} \mu \varepsilon^{-1}\end{array}$ & $\begin{array}{l}K \varepsilon \text { OSA } \\
10^{-6} \mu \varepsilon^{-1} \\
\end{array}$ & $\begin{array}{l}\% \\
\text { difference }\end{array}$ \\
\hline A-p1-02 & Polyamide & FOS 2 & 1560 & 0.779 & 0.781 & 0.3 \\
\hline \multirow{2}{*}{ D-II-pc-14 } & \multirow{2}{*}{ Polyamide } & \multirow{2}{*}{ FOS 2} & 1539 & 0.779 & 0.771 & -1.0 \\
\hline & & & 1545 & 0.774 & 0.774 & 0.0 \\
\hline \multirow{2}{*}{ D-II-pc-12 } & \multirow{2}{*}{ Polyamide } & \multirow{2}{*}{ FOS 2} & 1539 & 0.782 & 0.780 & -0.2 \\
\hline & & & 1545 & 0.776 & 0.770 & -0.8 \\
\hline \multirow{2}{*}{ D-I-pa-08 } & \multirow{2}{*}{ Polyamide } & \multirow{2}{*}{ FOS 2} & 1539 & 0.788 & 0.774 & -1.8 \\
\hline & & & 1545 & 0.776 & 0.779 & 0.4 \\
\hline
\end{tabular}

Table 4.9: Retest results on FOS2 with a spectrum analyzer 


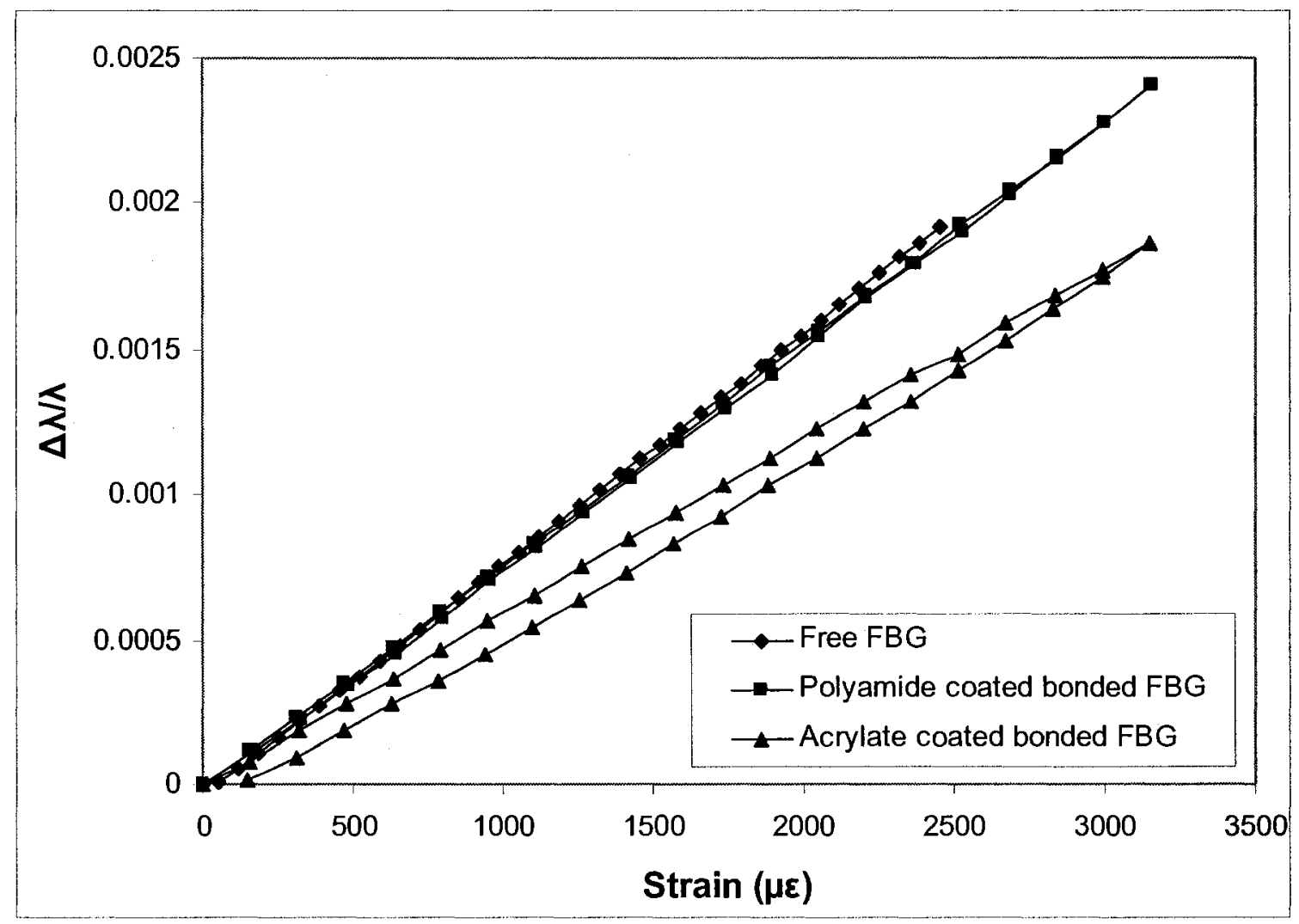

Figure 4.24: Strain sensitivity dependence on coating

\section{Strain Sensitivity Dependence on Coating}

As can be seen from Figure 4.24, when bonded the sensitivity of acrylic coated gratings was significantly less then the sensitivity of the polyamide coated gratings. The difference can be attributed to a significantly softer and thicker acrylate coating. According to Corning, Bennett et al., 2007 standard acrylic coatings are made to protect the grating and actually transfer as little strain as possible while polyamide coatings are hard and thin and therefore transfer strain very well. In general it is expected that epoxy and coatings will reduce the strain sensitivity of the grating, moreover the gauge length of the sensor will also affect the strain transfer $\mathrm{Li}$ et al, 2006. The results 
show that when compared to the free gratings, bonded polyamide and bare gratings had 1.4 to $5.2 \%$ lower sensitivity then the free gratings. Such decrease can be attributed to the strain transfer loss in the epoxy, but the actual transfer of the strain from the coating to the core is good. On the other hand bonded acrylic gratings had 23 to $31 \%$ lower sensitivity then free gratings, exhibited some hysterisis and overall wavelength drift as can be seen from Figure 4.24 and Figure 4.19. The decrease can be attributed to the combined effect of strain transfer loss in the epoxy and soft acrylic coating, with the later being the dominating effect.

\section{Strain Sensitivity Dependence on Configuration}

As in the case of free gratings, bonded serial configuration was slightly less sensitive then bonded arrayed (see Figure 4.21).

\section{Repeatability}

All gratings on FOS1 were also retested three times to check for the repeatability of the results. The comparison of the three trials is shown in Table 4.10

\begin{tabular}{|c|c|c|c|c|c|c|}
\hline $\begin{array}{l}\text { Grating } \\
\text { Index }\end{array}$ & Coating & Plate & $\begin{array}{l}K_{\varepsilon} \text { trial } 1 \\
10^{-6} \mu \varepsilon^{-1}\end{array}$ & $\begin{array}{l}K_{\varepsilon} \text { trial } 2 \\
10^{-6} \mu \varepsilon^{-1}\end{array}$ & $\begin{array}{l}K_{\varepsilon} \text { trial } 3 \\
10^{-6} \mu \varepsilon^{-I}\end{array}$ & $\begin{array}{l}\text { Max \% } \\
\text { difference }\end{array}$ \\
\hline A-p1-03 & Polyamide & FOS 1 & 0.772 & 0.770 & 0.772 & -0.271 \\
\hline A-p2-09 & Polyamide & FOS 1 & 0.764 & 0.765 & 0.766 & 0.271 \\
\hline A-a1-13 & Acrylic & FOS 1 & 0.561 & 0.529 & 0.505 & -9.891 \\
\hline$A-a 2-10$ & Acrylic & FOS 1 & 0.597 & 0.623 & 0.620 & 4.334 \\
\hline A-a $1-12$ & No Coating & FOS 1 & 0.752 & NA & NA & $\mathrm{NA}$ \\
\hline
\end{tabular}

Table 4.10: Re-test results on FOS1

As expected the polyamide gratings had very repeatable sensitivity with the \% difference not exceeding $0.3 \%$, on the other hand the maximum sensitivity difference for 
acrylic coated gratings was close to $10 \%$. The only grating for which the sensitivity could not be verified was $1540 \mathrm{~nm}$ bare grating. It appeared that the wave meter could not capture the peak data for all strain values and therefore the sensitivity could not be determined. When the grating was verified with a spectrum analyzer it appeared that a crack had developed and the spectrum now consisted of a superposition of a typical Fabry-Perrot interference pattern formed by a crack cavity and the grating itself. The spectrum is shown in Figure 4.25 the center wavelength of such peak is indeed difficult to determine with a wavemeter. This shows that even though good strain transfer can be achieved with bare gratings the reliability of such sensor is greatly reduced.

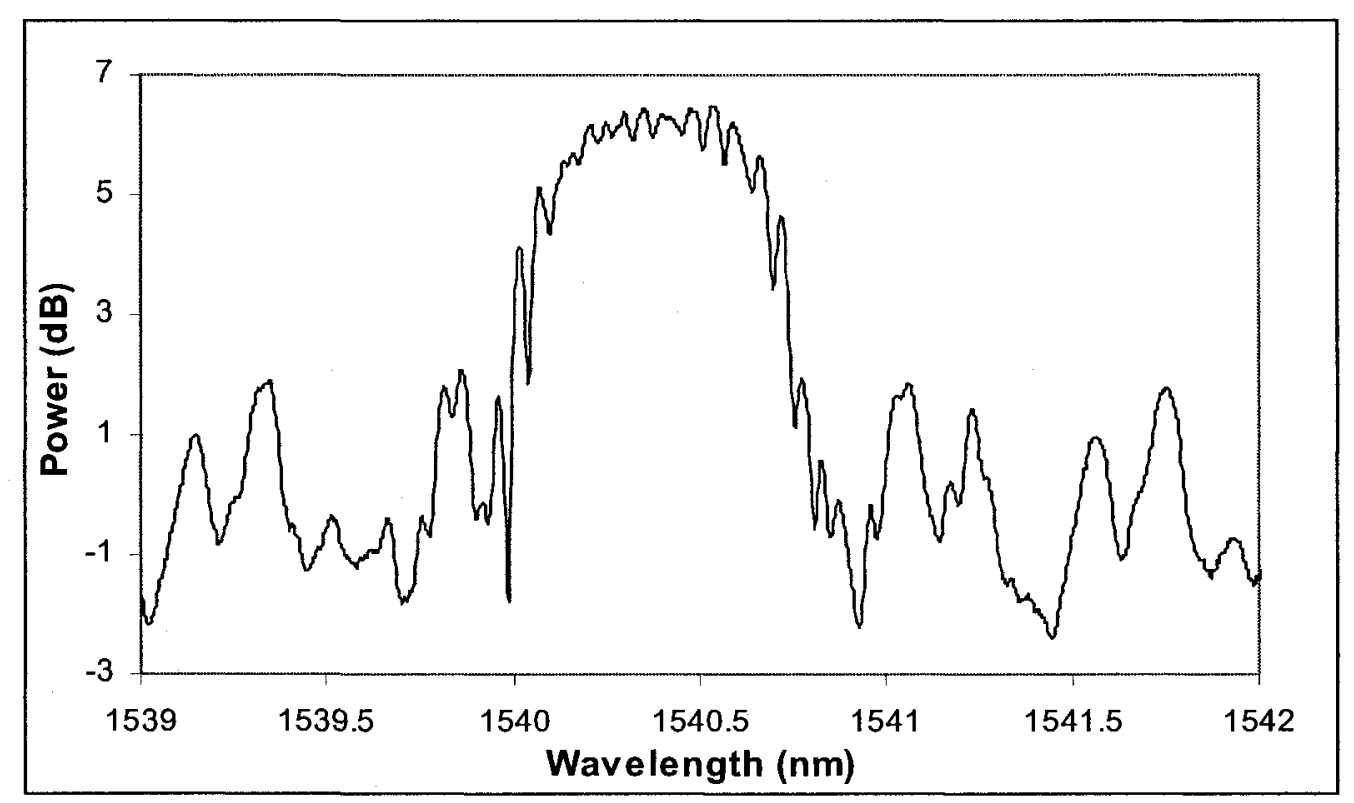

Figure 4.25: Reflection spectrum of a $1540 \mathrm{~nm}$ bare grating

\subsubsection{Bonded Temperature Characterization Procedure and Results}

Temperature characterization tests have been carried out using an ESPEC temperature chamber on both aluminium samples simultaneously. The samples were first heated from 
room temperature up to $80^{\circ} \mathrm{C}$ in $20^{\circ} \mathrm{C}$ increments and then cooled down to $-20^{\circ} \mathrm{C}$ in $15^{\circ} \mathrm{C}$ increments. To ensure the samples reached the desired temperature, strain gauge data from the two front strain gauges has been constantly monitored and the wavelength data was not collected until the strain gauges did not change more then $10 \mu \varepsilon$ in the last 10 minutes. The total for ramp and hold time was around 40 minutes. Once the temperature reached stable point the reflection spectrum has been collected using Micron Optics si720 Sensing analyzer and the peak data extracted the same way as has been done in section 4.2.2. To avoid frequent reconnection of the fibers JDS SB series switch has been used. The temperature sensitivity has been determined from the slope of the wavelengths vs. temperature plots, the same way as it has been done in section 4.2.2. The results are presented in Table 4.11 and graphically in Figure 4.26 and Figure 4.27.

\begin{tabular}{lllll}
\hline $\begin{array}{l}\text { Grating } \\
\text { index }\end{array}$ & Coating & Plate & $\begin{array}{l}\text { Wavelength } \\
(\text { nm })\end{array}$ & $\begin{array}{l}\boldsymbol{K}_{\boldsymbol{T}} \\
\mathbf{1 0}^{-6}{ }^{\circ} \boldsymbol{C}^{-1}\end{array}$ \\
\hline A-p1-02 & Polyamide & FOS 2 & 1540 & 25.6 \\
A-p1-03 & Polyamide & FOS 1 & 1540 & 25.9 \\
A-p2-09 & Polyamide & FOS 1 & 1560 & 25.6 \\
A-a1-13 & Acrylic & FOS 1 & 1540 & 21.0 \\
A-a2-10 & Acrylic & FOS 1 & 1560 & 23.9 \\
A-a1-12 & No Coating & FOS 1 & 1540 & 26.0 \\
& & & 1539 & 26.0 \\
D-II-pc-14 & Polyamide & FOS 2 & 1545 & 26.3 \\
& & & 1539 & 25.6 \\
D-II-pc-12 & Polyamide & FOS 2 & 1545 & 25.8 \\
& & & 1539 & 25.5 \\
D-I-pa-08 & Polyamide & FOS 2 & 1545 & 25.8 \\
\hline
\end{tabular}

Table 4.11: Bonded Temperature sensitivity results 


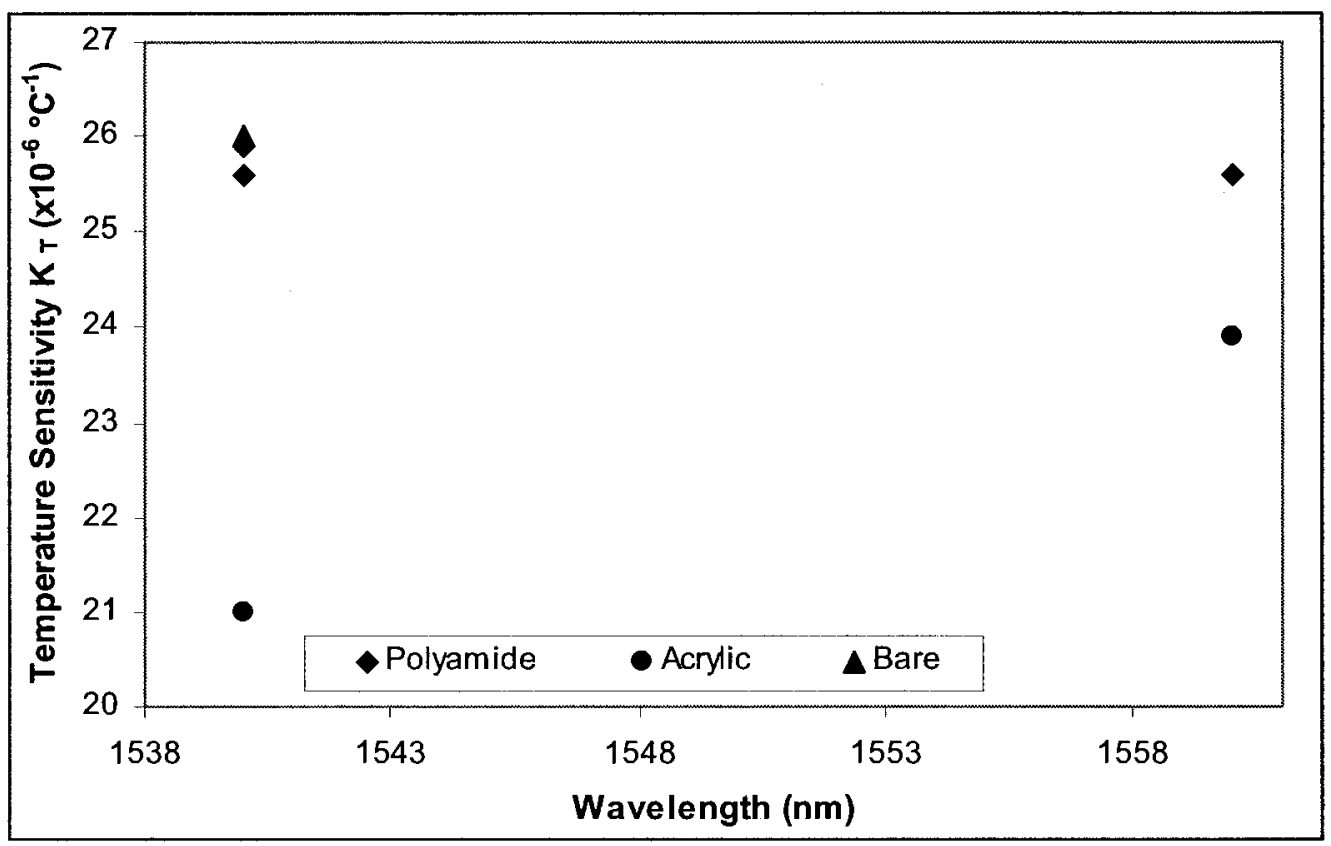

Figure 4.26: Bonded temperature sensitivity for single gratings

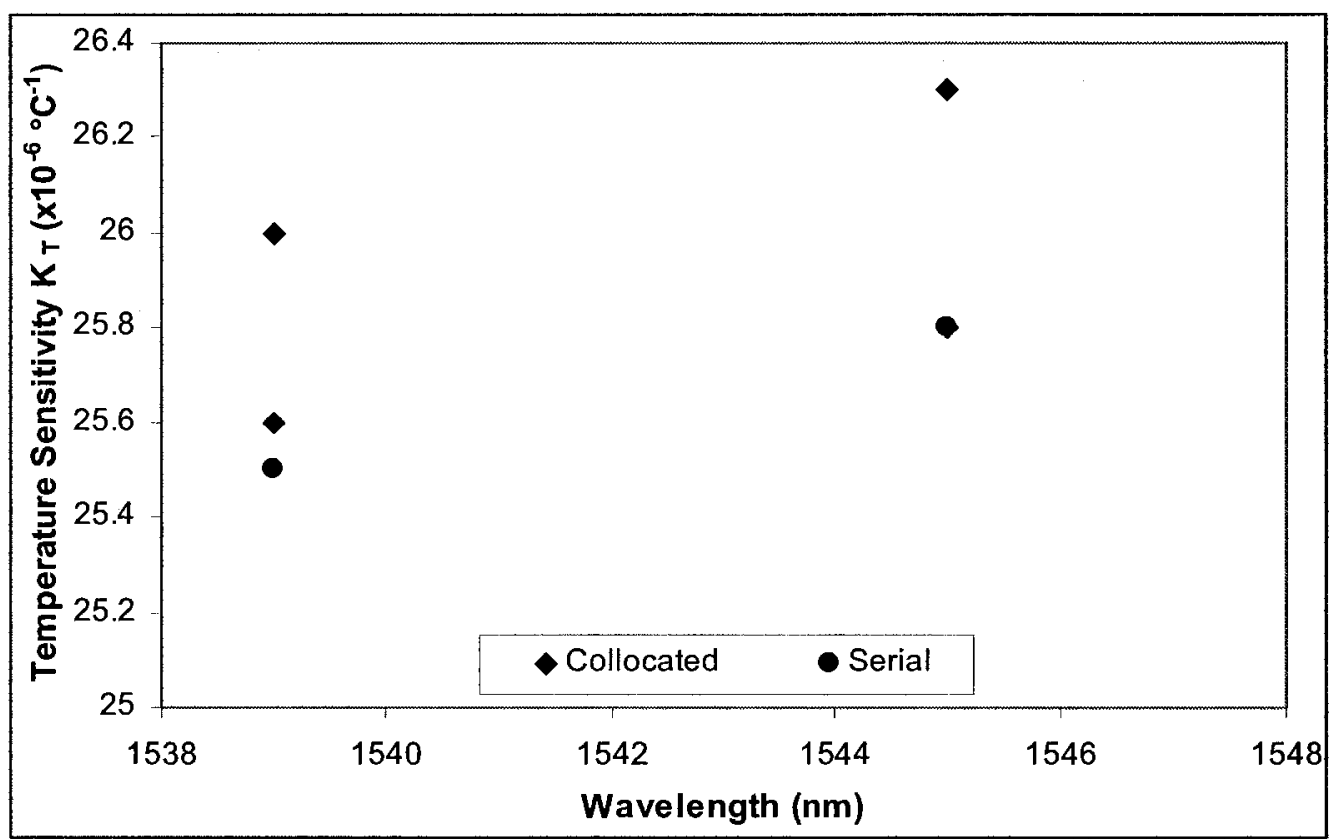

Figure 4.27: Bonded temperature sensitivity for dual gratings 
As can be seen from Table 4.11 Overall the temperature sensitivity is less then the theoretically predicted $30.52 \times 10^{-6}{ }^{\circ} \mathrm{C}^{-1}$. The discrepancy can be attributed to the incomplete thermal expansion induced strain transfer through the protective coatings.

\section{Temperature Sensitivity Dependence on Wavelength}

As can be seen from Figure 4.26 temperature sensitivity of single gratings did not display any apparent wavelength dependence. The reason is that bonding condition differences from grating to grating most likely cause much more variations then center wavelength. For dual gratings the higher wavelength grating did have a consistently higher sensitivity then lower wavelength grating. The difference is not large but is consistent with the free characterization results.

\section{Temperature Sensitivity Dependence on Coating}

Temperature sensitivity dependence on coating can be seen from Figure 4.26. Acrylic coated gratings displayed a consistently lower temperature sensitivity then bare and polyamide coated gratings. The lowest temperature sensitivity is reported for the 1540 acrylic coated A-a1-13 which also had the lowest strain sensitivity. Polyamide coated gratings had higher temperature sensitivity which is consistent because polyamide coating is harder and therefore transfers strain better.

\section{Temperature Sensitivity Dependence on Configuration}

As can be seen from collocated gratings didn't have a significantly more different temperature sensitivity than serial. 


\subsection{Summary}

In this chapter the sensitivity of free and bonded gratings to temperature and strain has been determined. The proceedings of the free characterization work have been presented at an international conference, Mrad et al. 2007. For free gratings it can be concluded that there is little dependence of the sensitivities on the center wavelength or configuration, however for very low temperatures the acrylic coating stiffens up and the sensitivity becomes highly non-linear. During the bonded tests it has also been determined that softer, acrylate coating does significantly reduce the strain sensitivity and therefore for bonded applications it is better to use polyamide coated gratings. Finally the low wavelength dependence of the sensitivities does not allow to discriminate between temperature and strain using just a pair of gratings with closely spaced center wavelengths. Therefore in order to effectively discriminate between temperature and strain using just a pair of gratings the sensor must be designed in such a way that either the temperature or strain sensitivity of one of the gratings is significantly different from that of the another. This can be done either by writing the gratings under different conditions such as exposure time and fiber hydrogenation, or by isolating one of the gratings from either temperature or strain. In both approaches system complexity and cost must be considered. 


\section{Chapter 5: Tilted Gratings, Vibration and Bend Sensing}

Currently there are two main limitations of tilted FBGs sensor designs. They take up a large wavelength span making multiplexing difficult and cladding modes can only be detected in transmission interrogation while in most cases it is easier to interrogate a sensor in reflection using the same fiber to carry the signal to and from the grating.

In the first part of this chapter the sensitivity of the individual cladding modes to bending will be studied in more detail to gain a better understanding of the processes involved. Specifically the orientation dependence of the sensitivity will be addressed and a sensor capable of discrimination between orthogonal directions of bending will be proposed. A proof of concept temperature independent strain gauge based on only the Ghost and the Bragg modes will also be demonstrated. In the second part a novel method to interrogate some of the cladding modes in reflection will be discussed and a proof of concept vibration sensor interrogated by an inexpensive power detector will be demonstrated.

\subsection{Tilted Grating Fabrication}

One centimetre long tilted gratings were inscribed in hydrogen-loaded Corning SMF28 fibers using a pulsed $\mathrm{KrF}$ excimer laser and the phase mask technique Hill et al., 1993, Lemaire et al., 1993. The grating period was $536 \mathrm{~nm}$ and the tilt angle ranged from $2^{\circ}$ to 
$4^{\circ}$. The tilt angle of the gratings affects the amount of power coupled from the $\mathrm{LP}_{01}$ core mode into the $\mathrm{LP}_{1 \mathrm{~m}}$ cladding modes. As shown in the theory section Figure 3.8 for the tilt angles ranging from $3^{\circ}$ to $6^{\circ}$ the coupling to the Bragg mode decreases much faster than the coupling to the cladding modes. For work in transmission the relative strength of the cladding modes and Bragg is unimportant, as long as they are detectable. Therefore for transmission experiments mainly $3^{\circ}$ and $4^{\circ}$ gratings were used.

\subsection{Cladding Mode Sensitivity to Bending, in Transmission.}

As discussed in the theory section bending asymmetrically changes the refractive index profile of the cladding modes, which changes the coupling to the cladding modes, affects the center wavelength and causes new modes to appear at nearby wavelengths. The dominating effect though is the change in power coupling. Preliminary results demonstrated that cladding modes experience several $\mathrm{dB}$ of power variation which depends on the orientation of bending and the position of the cladding mode relative to the Bragg. Typically lower order modes are more sensitive than higher order modes with the exception of the ghost mode which is only sensitive to small radius bending. Therefore in the following sections the analysis has been broken down into three curvature ranges: $\infty$ to $0.2 \mathrm{~m}$ curvature radii sensing using cladding modes located $2.42 \mathrm{~nm}$ away from the Bragg, 0.5 to $0.06 \mathrm{~m}$ curvature radii sensing using cladding modes located $5.71 \mathrm{~nm}$ away from the Bragg, and finally 0.04 and $0.025 \mathrm{~m}$ curvature radii sensing using the ghost mode located $1.75 \mathrm{~nm}$ away from the Bragg.

The advantage of using cladding modes a fixed distance away from the Bragg is that the measurements can be made temperature insensitive since temperature shifts the Bragg 
and the cladding modes by the same amount without affecting the power coupling to either of the modes. In contrast to the previous work the systematic study of the orientation dependence of the bending is performed and the repeatability issues are addressed. Finally a practical sensor which can be multiplexed and can take into account the temperature effects will be demonstrated based on the ghost mode alone.

\subsubsection{Large Curvature Bending Experimental Set-up.}

There are a number of approaches to implementing a fiber bending experiment. The most important issue is accurately measuring the curvature radii ranging form several meters down to several centimetres. The most common set-up is implemented by freely attaching a fiber to a thin elastic plate such that no strain is induced while the fiber is bent. Bending of a plate can then be done using either four-point Zhao et al., 2004 or three-point Rao et al., 2003 bending set-up. In this work a combination of the techniques has been used to achieve large bending range.

The fiber was taped to the surface of a thin piece of metal with the dimensions of $152.4 \times 11.45 \times 0.4 \mathrm{~mm}$. The experimental set-up is shown in Figure 5.1. The bend was induced using an $l$-translation stage and measured using an $l, h$-translation stage. The curvature radius was calculated using equation 5.1, Liu et al., 2000.

$$
R=\frac{\Delta h}{2}+\frac{\Delta l^{2}}{2 \Delta h}
$$




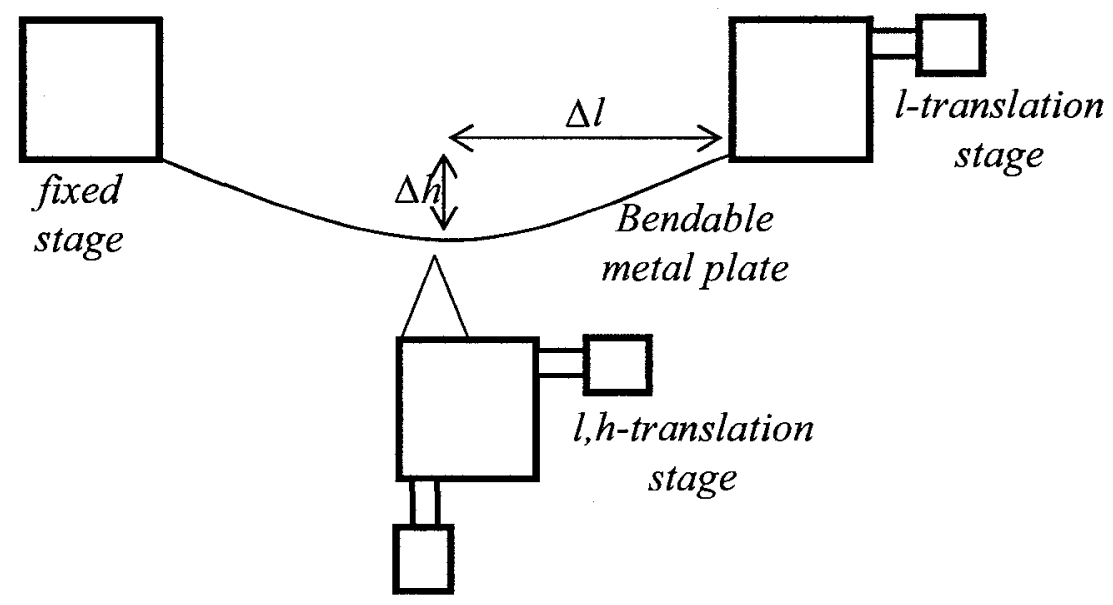

Figure 5.1: Bending set up

With this set up, curvatures ranging from $1 \mathrm{~m}$ to $10 \mathrm{~cm}$ can be induced in the $5 \mathrm{~cm}$ middle section and measured accurately. For large curvatures (between $1 \mathrm{~m}$ and $0.2 \mathrm{~m}$ ) the whole beam is approximately circular the $l$-translation stage is used to measure $\Delta l$ and the $l, h$-translation stage to measure $\Delta h$. For smaller curvatures only the middle section is circular since the beam has finite rigidity and eventually starts to fold as $\Delta l$ is reduced. Therefore for small curvature the $l, h$-translation stage is used to measure both $\Delta l$ and $\Delta h$ on the middle $5 \mathrm{~cm}$ section only. The fiber is taped using thin strips of masking tape on either side of the grating. This is done so that the grating slips a little bit as the curvature is decreased and no significant strain is induced in the fiber. Due to the way the gratings are written, the orientation of the bending is also very important. From Figure 3.1 it can be seen that the grating planes are only tilted in the $x-z$ plane while there is no tilt in the $y-z$ plane. Therefore the bending in those respective directions is also expected to 
produce different results. To keep track of the bending directions glass slides have been glued to the fiber in the $\mathrm{x}-\mathrm{z}$ plane prior to inscription.

\subsubsection{Large Curvature Bending $\infty>\mathrm{R}>0.2 \mathrm{~m}$.}

The spectrum evolution of the lower order cladding modes for bending radii in the range of $\infty$ to $0.2 \mathrm{~m}$ in the $\mathrm{x}-\mathrm{z}$ plane is shown in Figure 5.2 while the $\mathrm{y}-\mathrm{z}$ plane bending is shown in Figure 5.3.

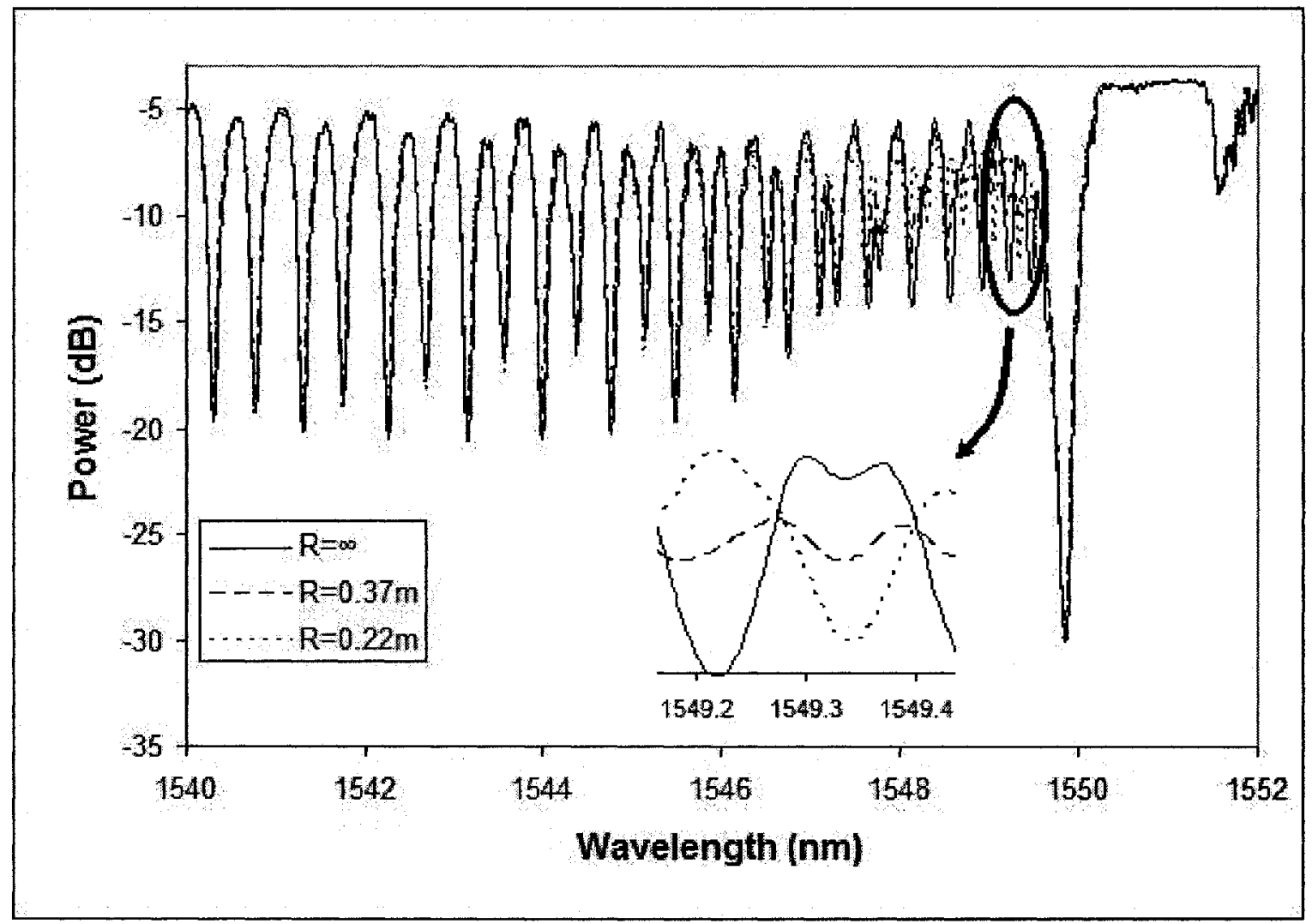

Figure 5.2: Large $R$ bending, $x-z$ plane bending 


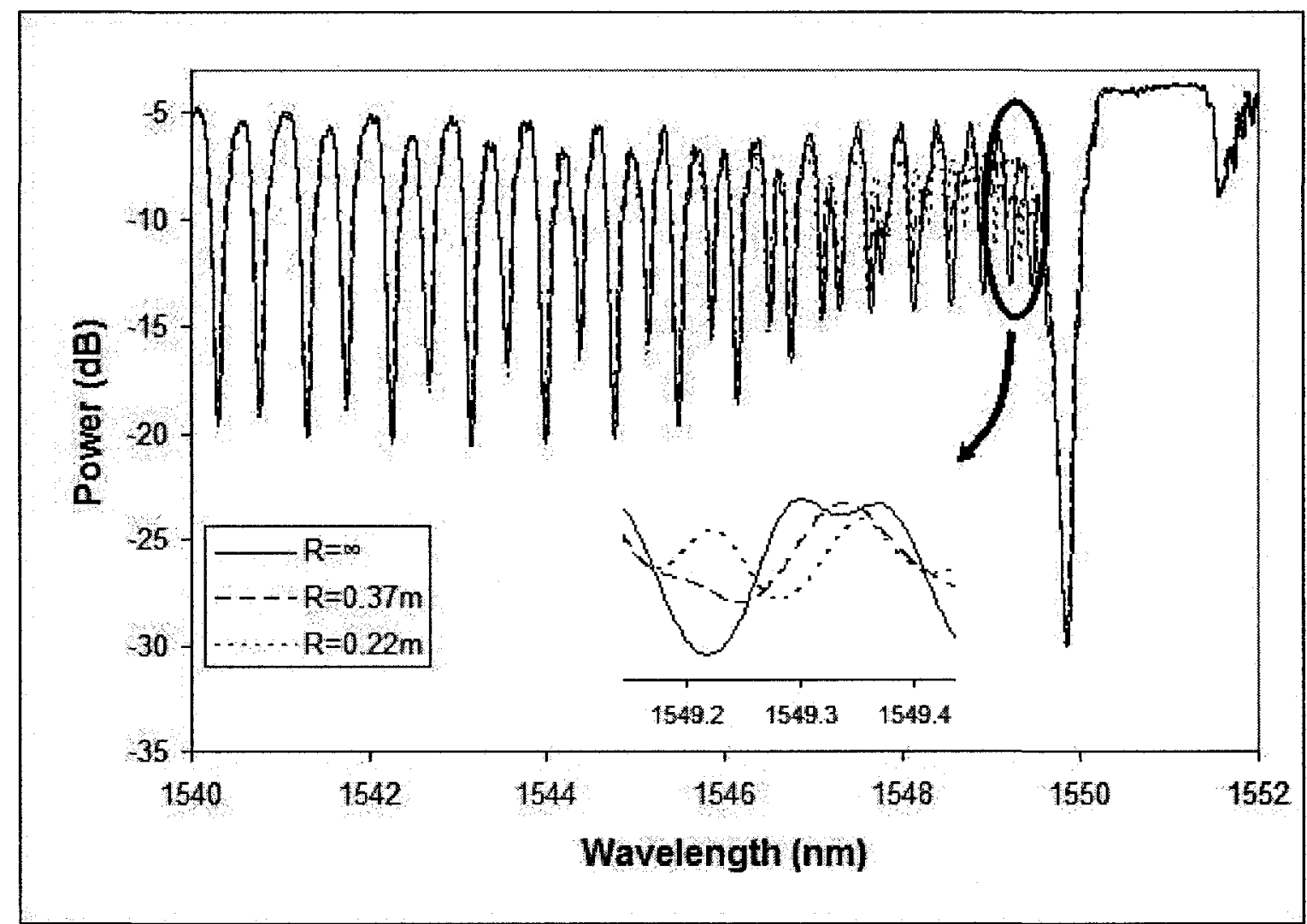

Figure 5.3: Large $R$ bending, $y$-z plane bending

The transmission spectrums were collected using the Micron Optics Si720 Sensing Analyzer. As can be seen from the figures there is a number of low order modes that undergo power coupling variation during bending. To increase the accuracy of the results all of the modes should be used however not all of them are well pronounced and to keep the analysis simple only two peaks will be considered. The small peak at $1549.35 \mathrm{~nm}$ representing the $\mathrm{LP}_{0,5}$ mode and a larger peak at $1549.218 \mathrm{~nm}$ representing the $\mathrm{LP}_{1,5}$ mode. When bending in the $\mathrm{x}-\mathrm{z}$ plane the large peak decays and experiences a shift towards smaller wavelengths. The smaller peak grows and shifts slightly to higher wavelengths. However when bending in the y-z plane the large peak splits into two distinct peaks that slightly spread in wavelength while the small peak disappears. 
To quantify the bend sensitivity, the normalized power within a $20 \mathrm{pm}$ window located 2.42nm away from the Bragg is plotted versus the curvature radius in Figure 5.4. $0^{\circ}$ and $180^{\circ}$ orientations correspond to the $\mathrm{x}-\mathrm{z}$ axis bending while $90^{\circ}$ and $270^{\circ}$ correspond to the $y-z$ axis bending. It can be seen that $x-z$ axis bending results in a significant power decrease while $y-z$ axis bending just contributes to the noise in the measurements. This demonstrates that by monitoring power in a small wavelength window centered on a predefined cladding mode a direction sensitive bend sensor can be implemented. The peaks are detectable for curvature radii up to $0.2 \mathrm{~m}$, past that they are more difficult to resolve since lower order modes are spaced very closely to each other and too many new modes start to appear. Therefore for smaller curvature radii higher order cladding modes should be used.

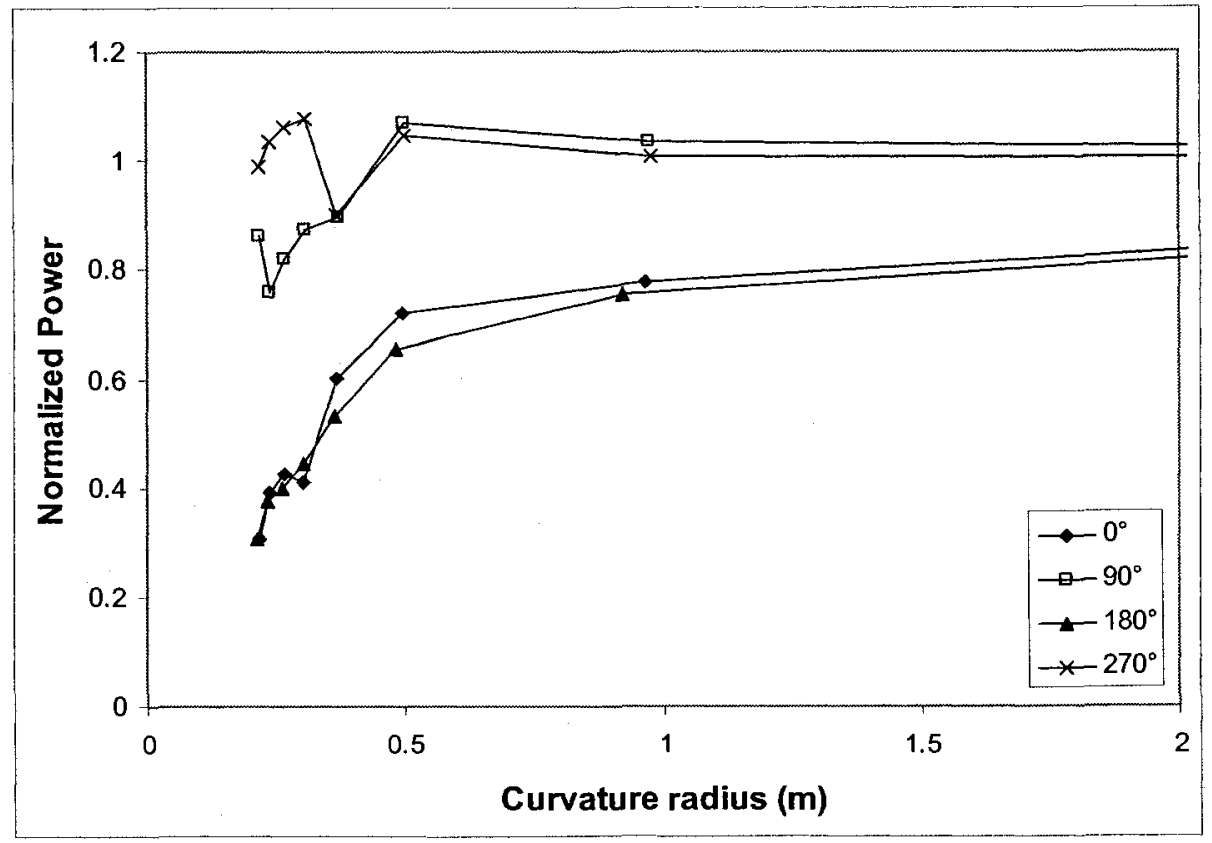

Figure 5.4: Cladding mode coupling sensitivity versus bending for two different orientations 


\subsubsection{Medium Curvature Bending 0.5 $>\mathbf{R}>0.06 \mathrm{~m}$.}

Figure 5.5 shows the evolution of the grating spectrum for the curvature radius reaching $0.066 \mathrm{~m}$ in the $\mathrm{x}-\mathrm{z}$ plane. It can be seen that coupling strength increases or decreases alternatively for higher order cladding modes more then $5 \mathrm{~nm}$ away from the Bragg. One mode decays the next one grows, and so on. On the contrary, in the y-z plane all modes decay, see Figure 5.6.

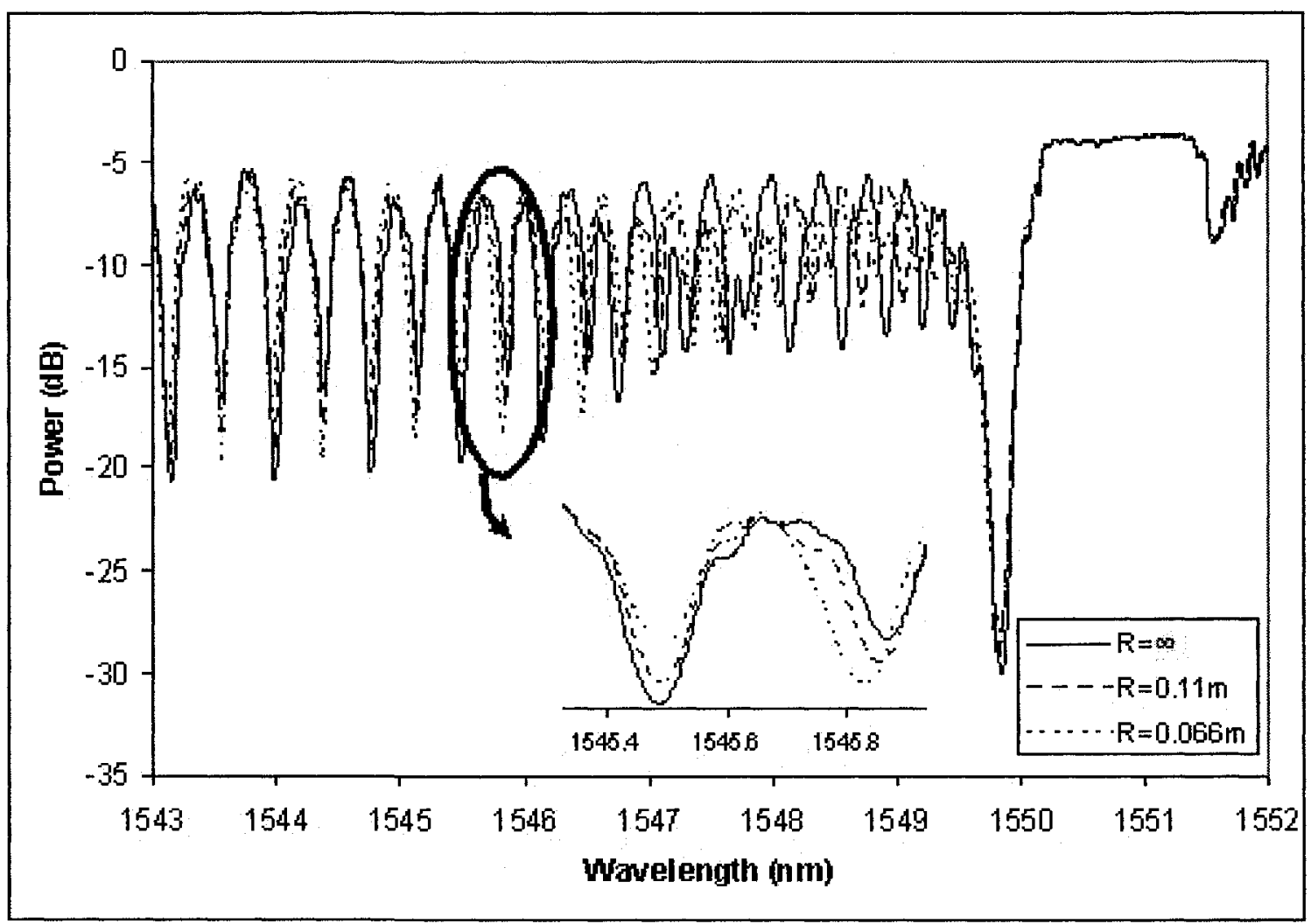

Figure 5.5: Large $R$ bending, in the $x-z$ plane 


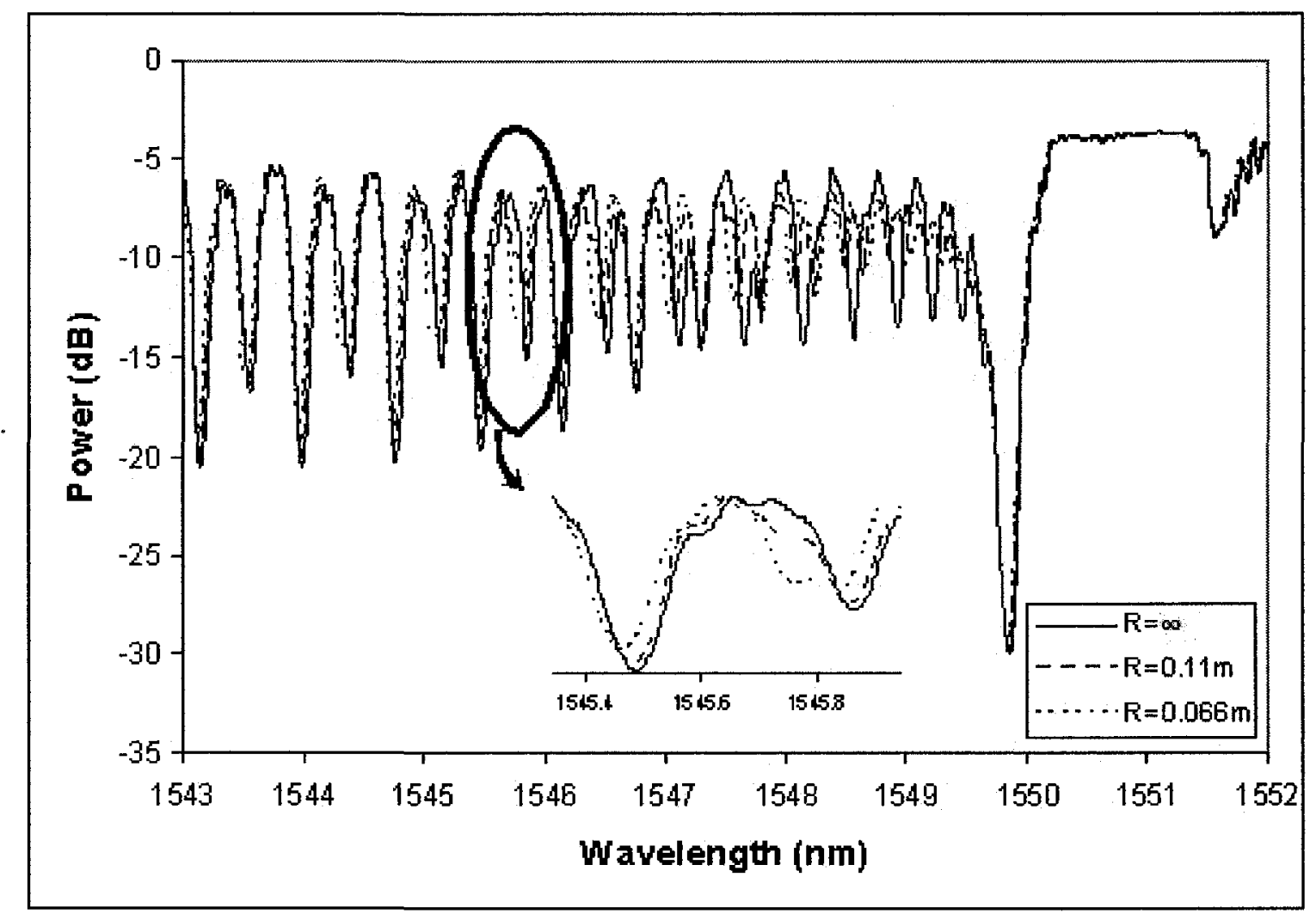

Figure 5.6: Large $R$ bending, in the $y$-z plane

Therefore again, by proper mode selection an orientation sensitive bend sensor can be implemented. To verify that this result can be repeated a second 4 degree grating has been tested, the writing conditions were kept the same however the spectrum did turn out different as shown in Figure 5.7. 


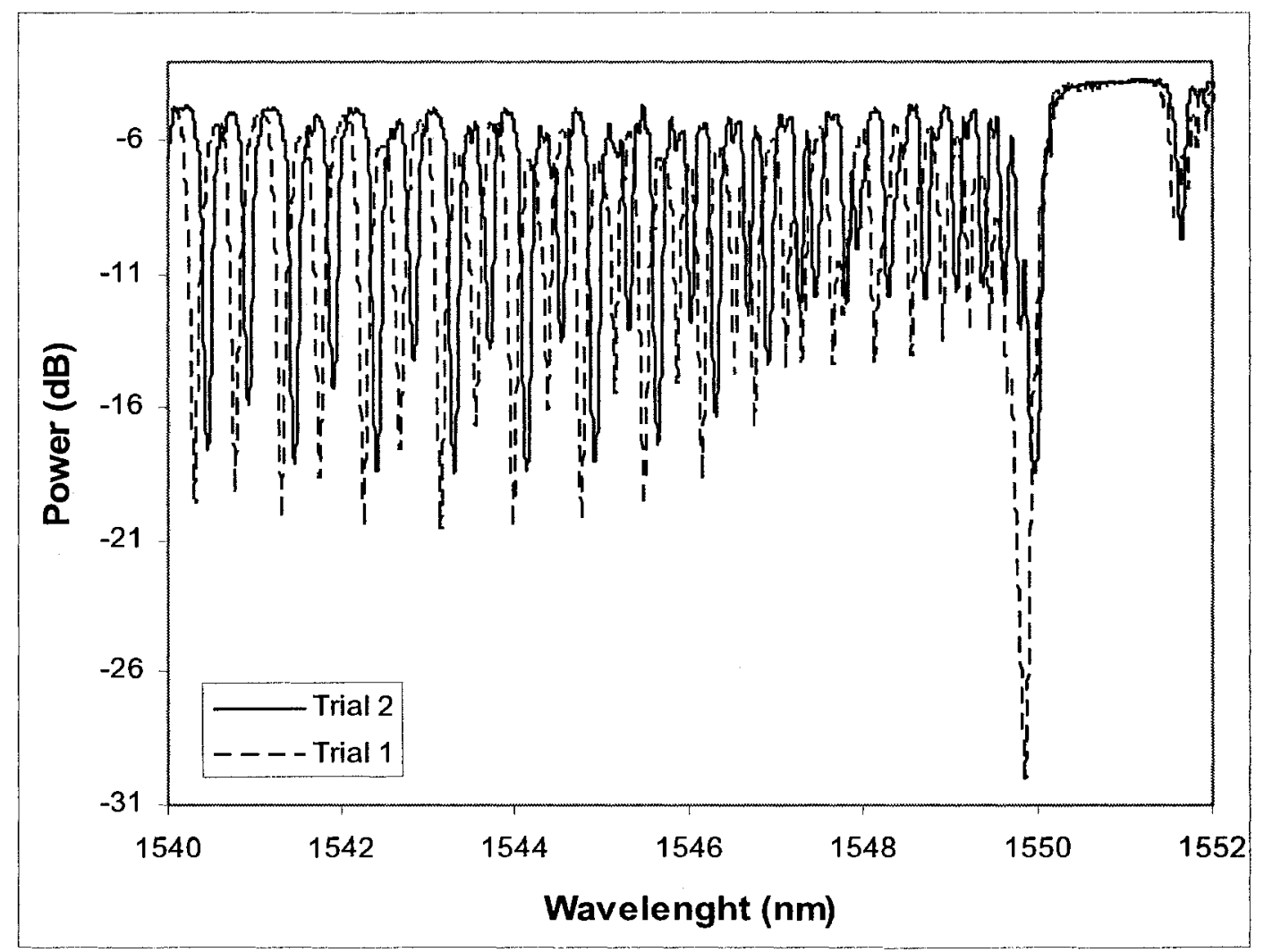

Figure 5.7: Repeatability comparison of the two spectrums

The strength of the Bragg modes was the same for both gratings however trial 2 had a clean single Bragg notch while trial 1 had multiple notches indicating that the grating was chirped during the writing conditions. The ghost mode strength was also very different for the two trials. The ghost mode for trial 1 was $26 \mathrm{~dB}$ while the ghost mode for trial 2 was only $15 \mathrm{~dB}$. The discrepancy can be attributed to either a tilt angle miss match or a wider Bragg mode of trial 1 which caused too many ghost modes to overlap. The higher order cladding modes turned out relatively the same for both trials with the cladding modes of trial 1 being slightly deeper. 
To compare the bending sensitivity of the gratings a cladding mode centered at $1545.86 \mathrm{~nm}$ and located $5.71 \mathrm{~nm}$ away from the Bragg was selected for trial 1 grating and the mode centered at 1546.02 and located $5.63 \mathrm{~nm}$ away from the Bragg was selected for trial 2. The bending sensitivity for trial 1 grating is shown in Figure 5.8 while the sensitivity of trial 2 is shown in Figure 5.9. It can be seen that both trials exhibit the same orientation dependence. The $x-z$ plane bending results in the mode growth while the $y-z$ bending results in the mode decay. The magnitude is slightly different though. The maximum peak shift for trial 1 was $3 \mathrm{~dB}$ while for trial 2 it was only $2 \mathrm{~dB}$. The difference might be attributed to the experimental setup repeatability errors as well as the differences in the spectrum profiles. But in general it can be concluded that the results are repeatable.

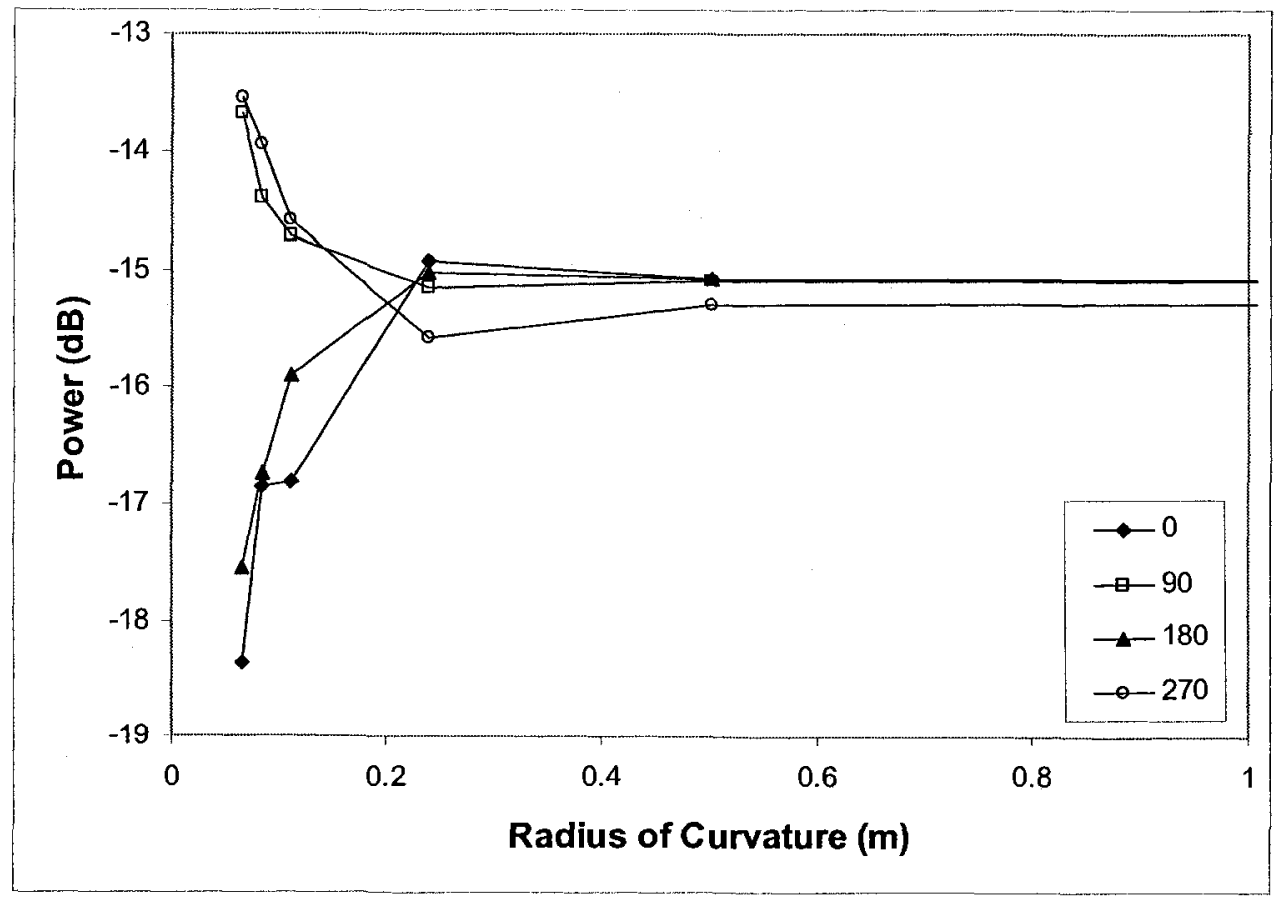

Figure 5.8: Trial 1 bending vs. curvature 


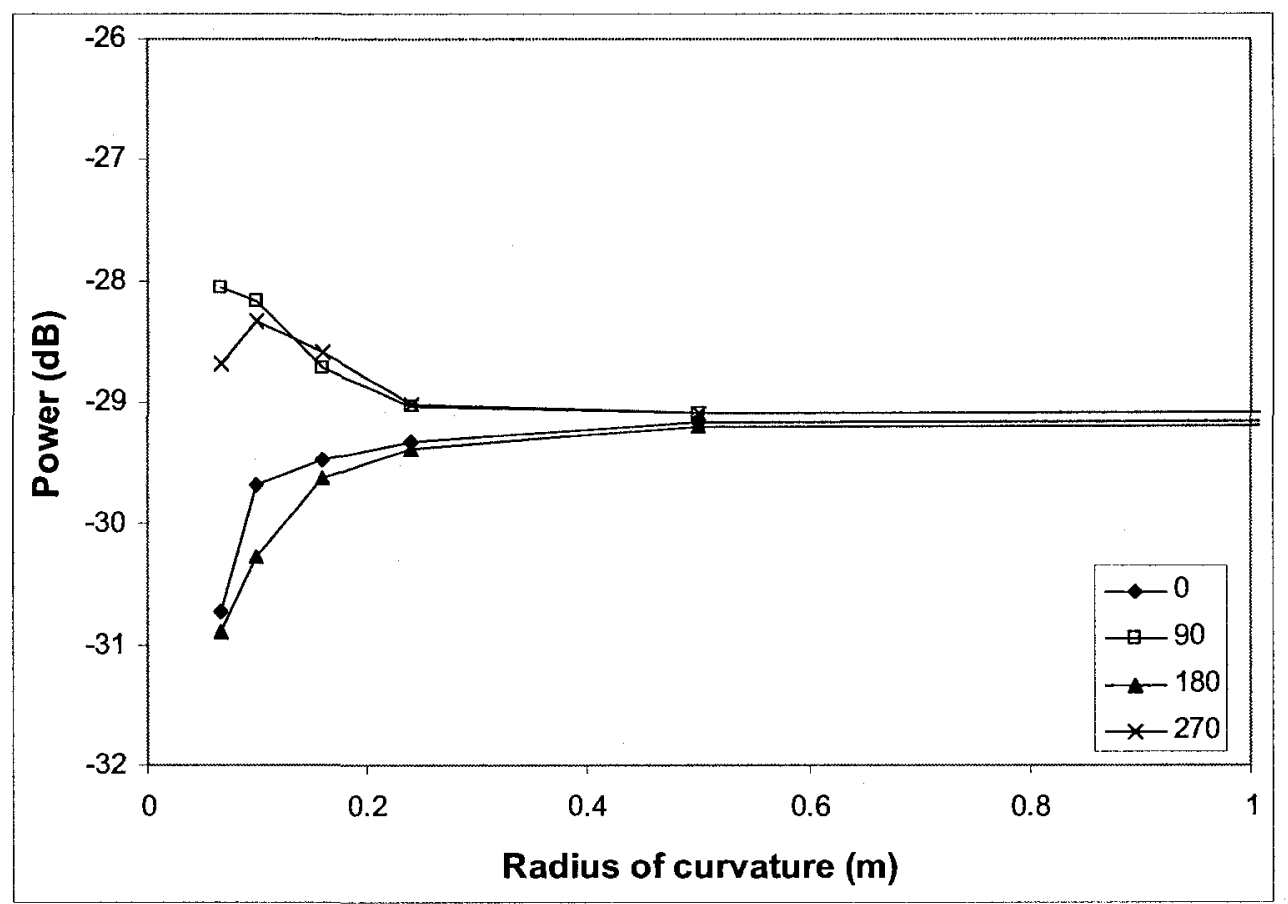

Figure 5.9: Trial 2 bending vs. curvature

\subsubsection{Small Curvature Bending 0.05m $>R>0.02 \mathrm{~m}$.}

Another cladding mode which is of a particular interest in bending experiments is the ghost mode. As mentioned earlier it is the first peak after the Bragg and consists of a number of cladding modes. As can be seen from Figure 5.5 and Figure 5.6 it is not particularly sensitive to bending up to the curvatures of $0.066 \mathrm{~m}$ however it does become very sensitive for smaller radii. Since the beam bending set-up was not suitable any more for such small bending radii the bending was done by looping the fiber once and pulling on the two free ends using translation stages. Figure 5.10 shows the evolution of the ghost mode of a 3 degree titled grating around $44 \mathrm{~mm}$ radii. Figure 5.11 shows the evolution for radii around $27 \mathrm{~mm}$. In this experiment the orientation was not controlled. 


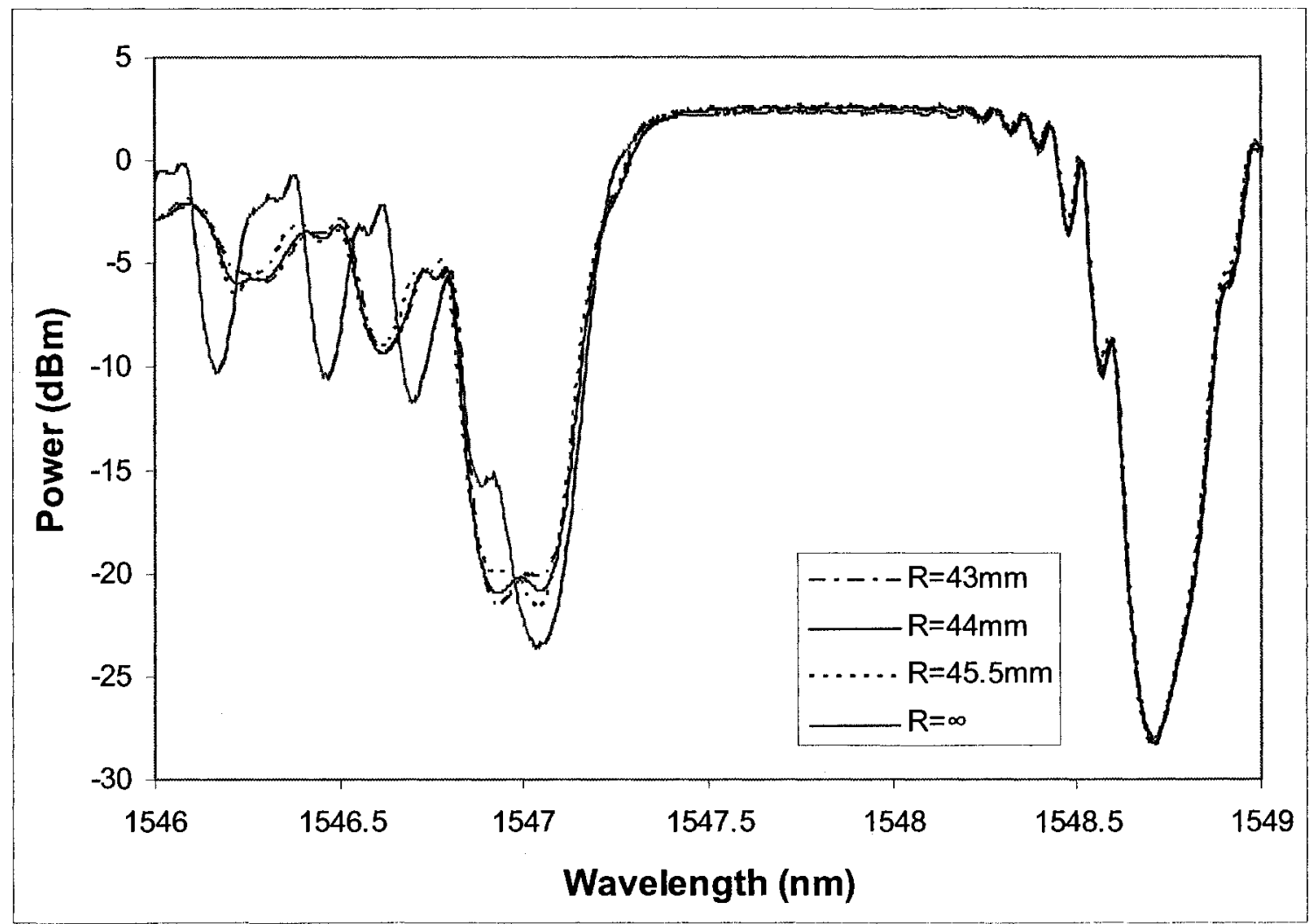

Figure 5.10:Transmission spectrum for the curvature ranging from 45.5 to $43 \mathrm{~mm}$

As mentioned in the theory, bending changes the refractive indices and coupling coefficients of the cladding modes. The Ghost mode itself is made up of a number of cladding modes. Therefore as the fiber is bent the ghost mode is swept by a number of modes that alternate in transmission notch depth. This results in apparent splitting of the Ghost mode into two notches one of which becomes shallower and another deeper as the bending radius changes. 


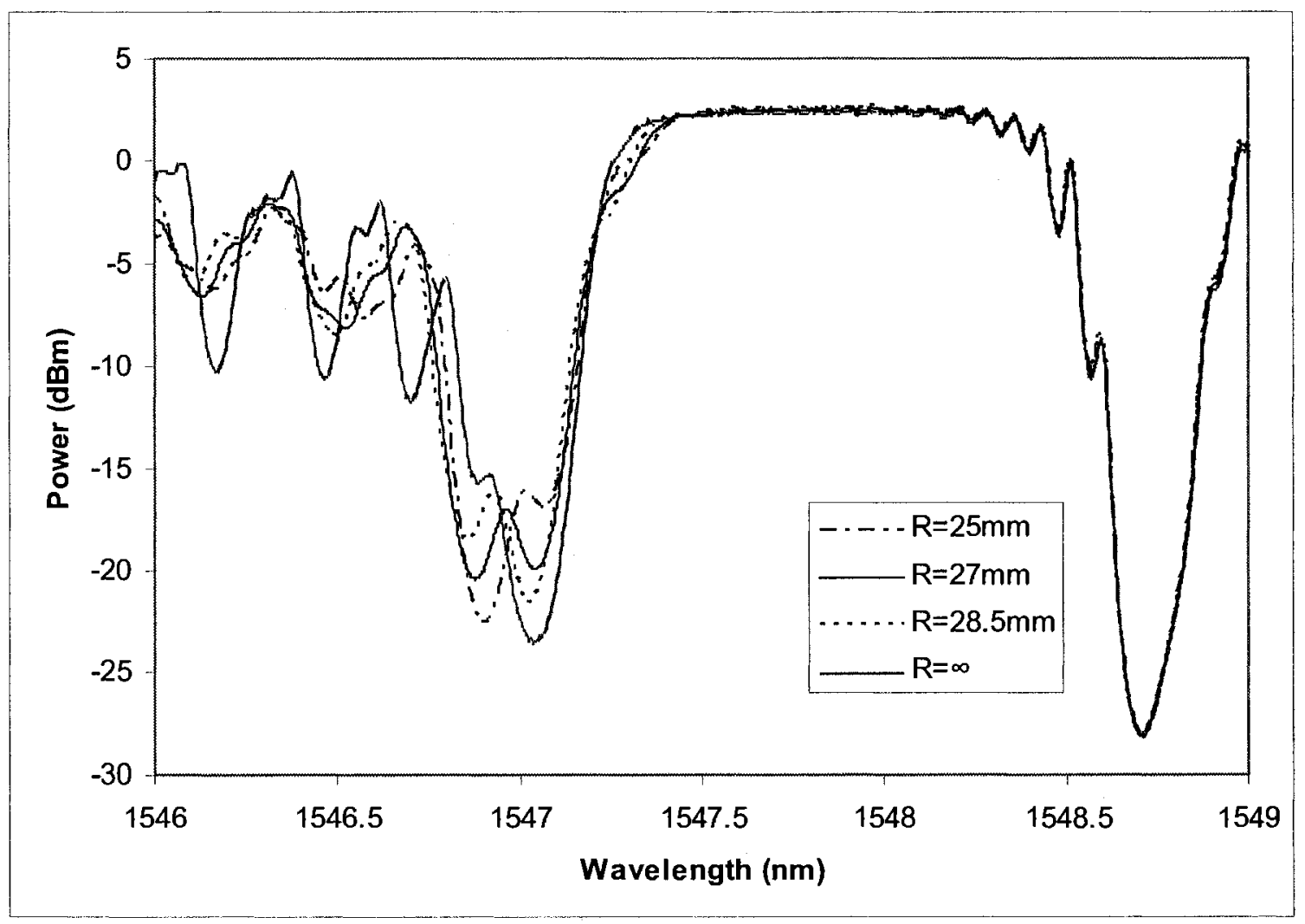

Figure 5.11:Transmission spectrum for the curvature ranging 28.5 to $25 \mathrm{~mm}$

In Figure 5.10 the ghost mode splits for the curvature radius of $44 \mathrm{~mm}$ in Figure 5.11 the ghost mode splits for the curvature radius of $27 \mathrm{~mm}$. To verify if the splitting is affected by the temperature, the fiber was looped with a $27 \mathrm{~mm}$ radius and placed on top of a Peltier heater plate. The heater temperature was then raised from $23^{\circ} \mathrm{C}$ up to $70^{\circ} \mathrm{C}$ and then lowered down to $-6^{\circ} \mathrm{C}$. As predicted in section 3.3.2 the whole spectrum shifted in wavelength as the grating was cooled from $70^{\circ} \mathrm{C}$ down to $-6^{\circ} \mathrm{C}$, however the depth of the Bragg and the Ghost mode remained the same. The experimental data is shown in Figure 5.12 where all of the traces are centered on the $-6^{\circ} \mathrm{C}$ trace. 


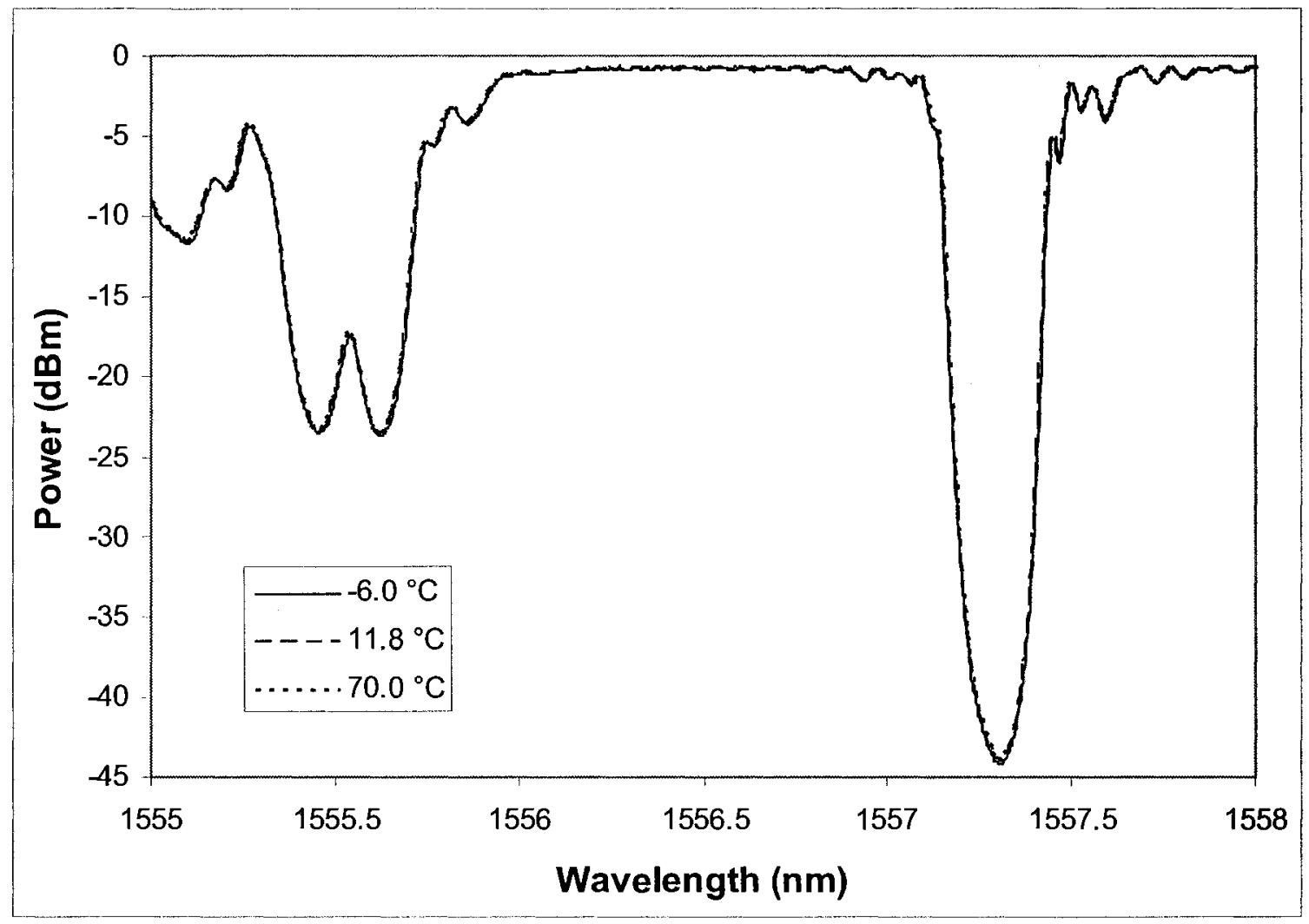

Figure 5.12:Temperature sensitivity of a TFBG coiled with a $27 \mathrm{~mm}$ radius

To verify the orientation dependence and repeatability again two gratings with marked tilt plane orientations have been used. Figure 5.13 and Figure 5.14 show the ghost mode transmission spectrum evolution for curvatures radii around $27 \mathrm{~mm}$ and two different orientations. It can be seen that for the $\mathrm{x}-\mathrm{z}$ orientation splitting can easily be observed but for the $y-z$ orientation the ghost mode remains essentially unchanged. To illustrate the difference ghost peak power vs. bending for four different orientations is plotted in Figure 5.15. 


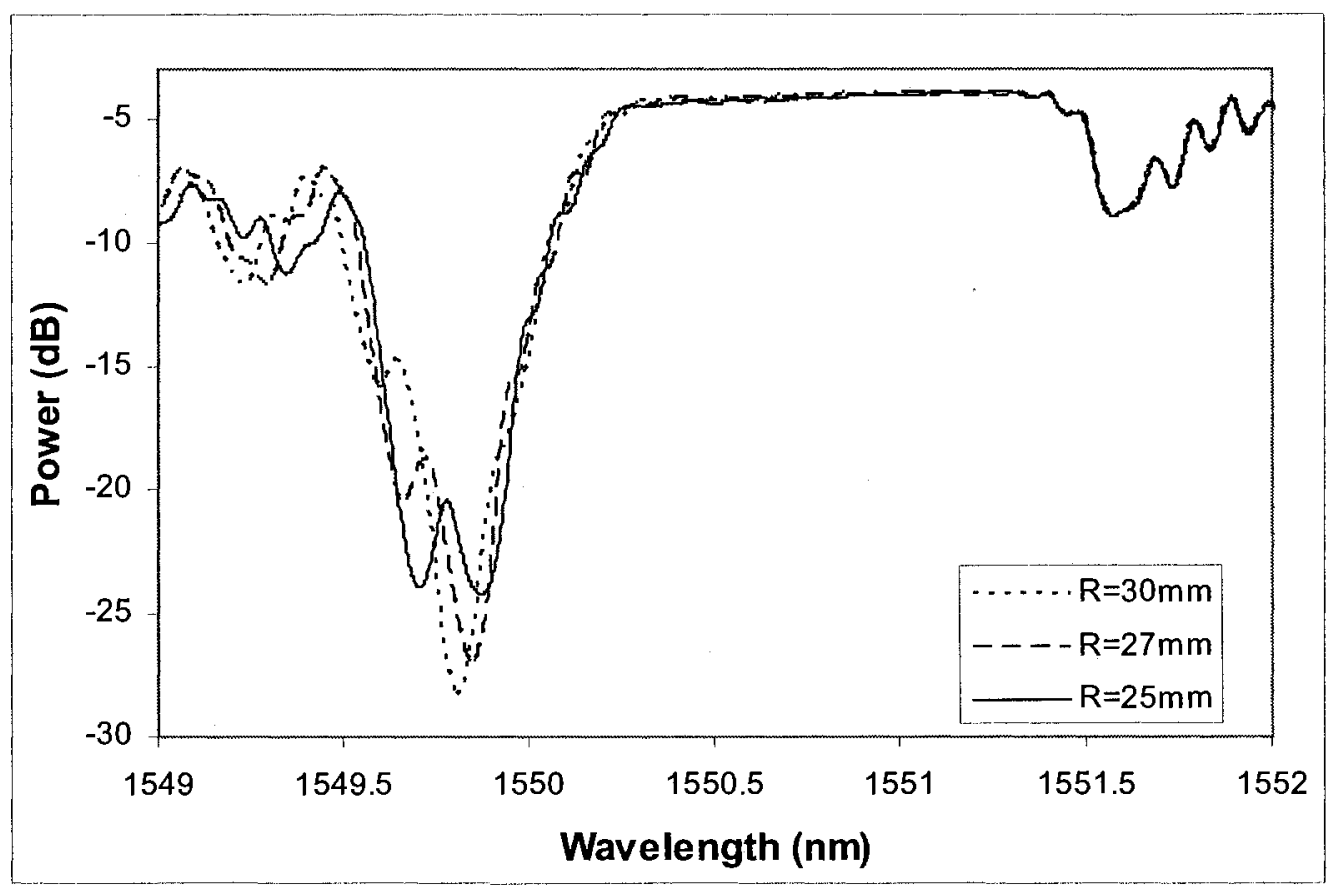

Figure 5.13: Ghost mode bending sensitivity $x-z$ plane

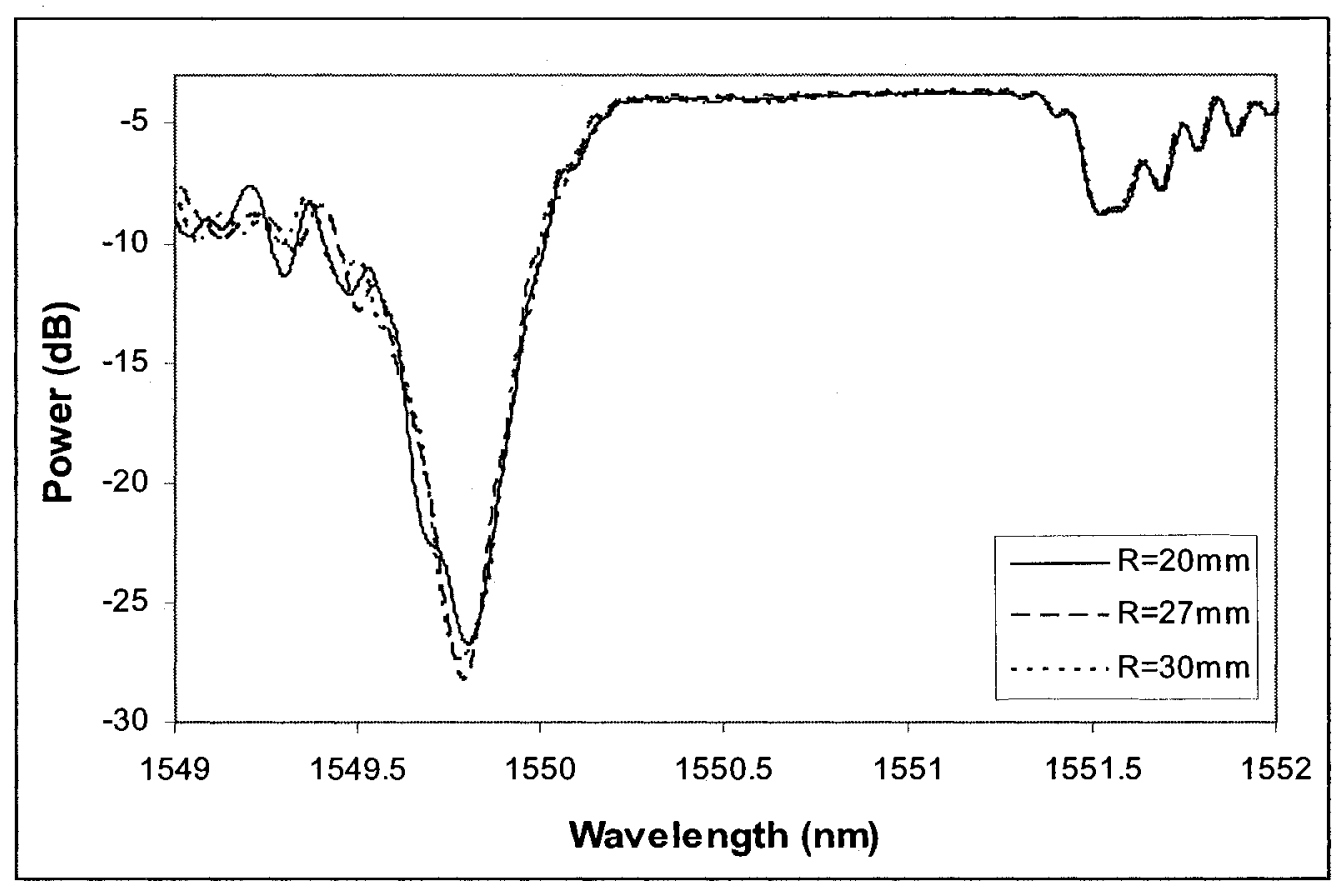

Figure 5.14: Ghost mode bending sensitivity $y-z$ plane 


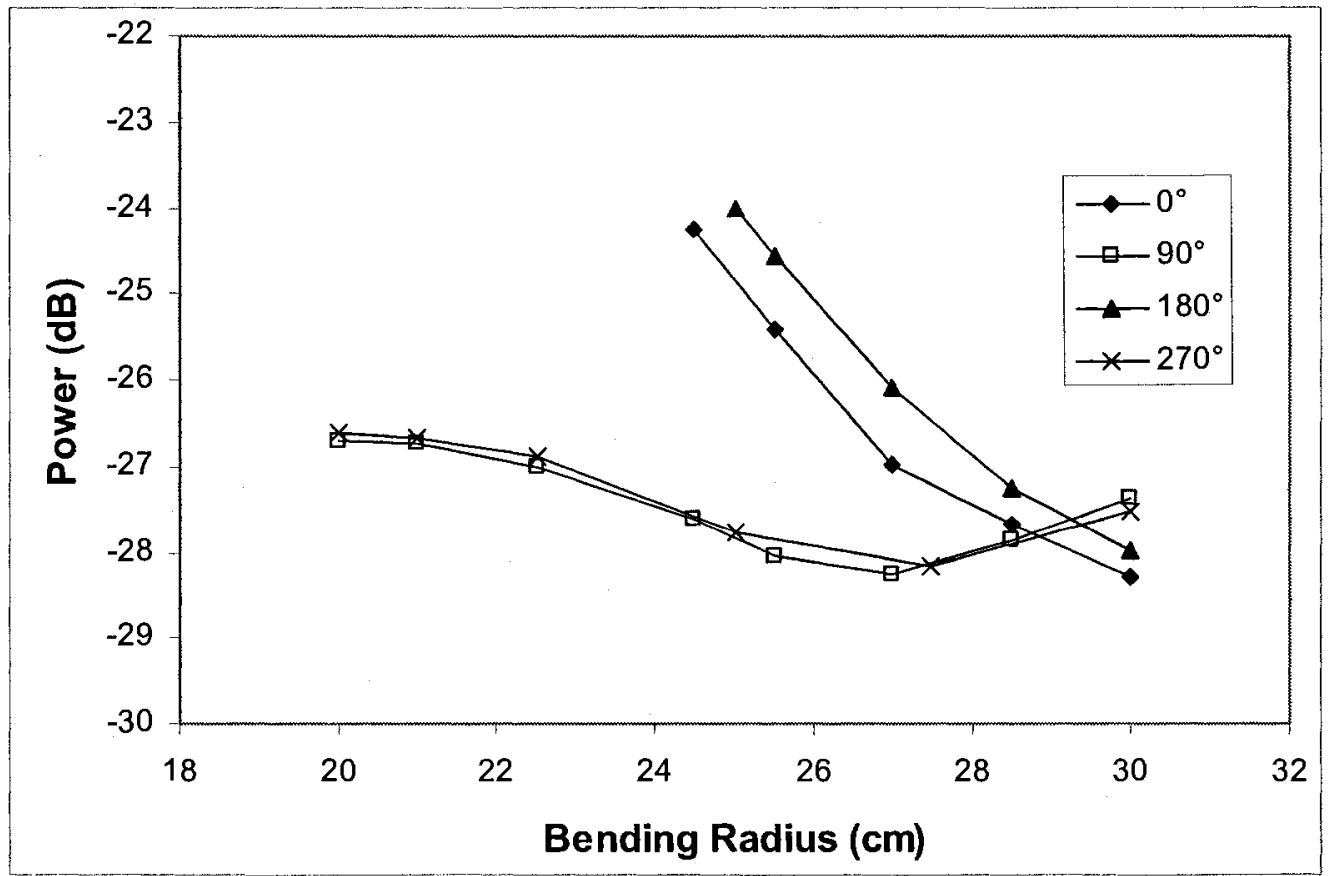

Figure 5.15: Ghost mode power coupling vs. the bending radius

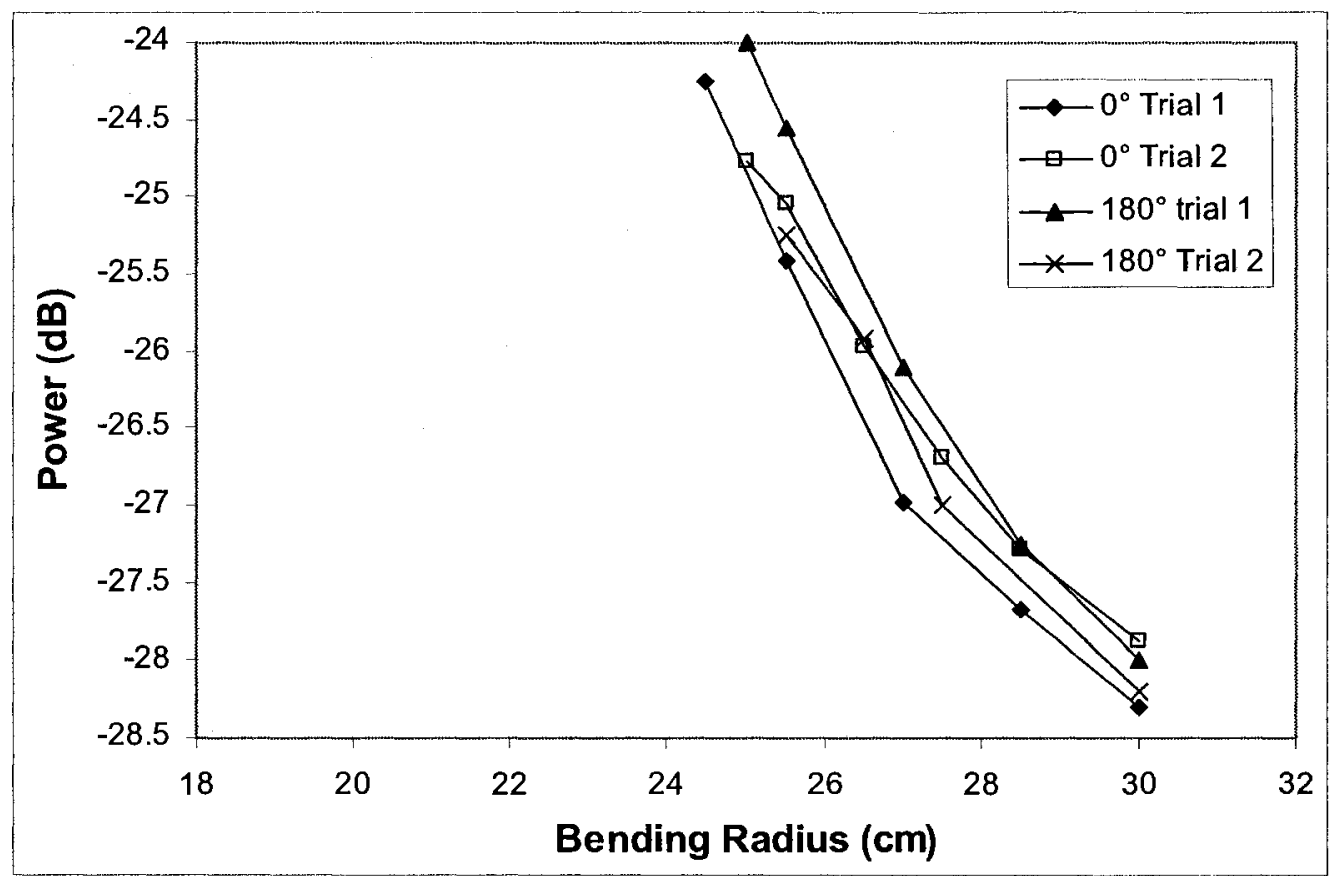

Figure 5.16: Ghost mode power coupling vs. the bending radius for two different gratings 
From Figure 5.15 it can be seen that again the $0^{\circ}$ and $90^{\circ}$ orientations which are corresponding to the $\mathrm{x}-\mathrm{z}$ plane had the highest sensitivity to bending. When repeated on a trial 2 grating the sensitivity to bending was also very similar as can be seen from Figure 5.16 proving that the results are repeatable and do not depend significantly on the grating profile.

\subsubsection{Proof of Concept, Temperature Independent Strain Gauge Demonstration.}

To demonstrate the capability of measuring strain with such sensor a fiber containing a grating was bonded to an ASTM E8M $6061 \mathrm{~T} 6$ aluminum substrate. Bonding was done with a room temperature curable epoxy. The fiber was bent with the curvature radius of $24.8 \mathrm{~mm}$ and bonded in two spots $26.42 \mathrm{~mm}$ away from each other as shown in Figure 5.17. The opposite side of the substrate was outfitted with the Vishay CEA-06-240UZ120 piezo-resistive strain gage for reference.

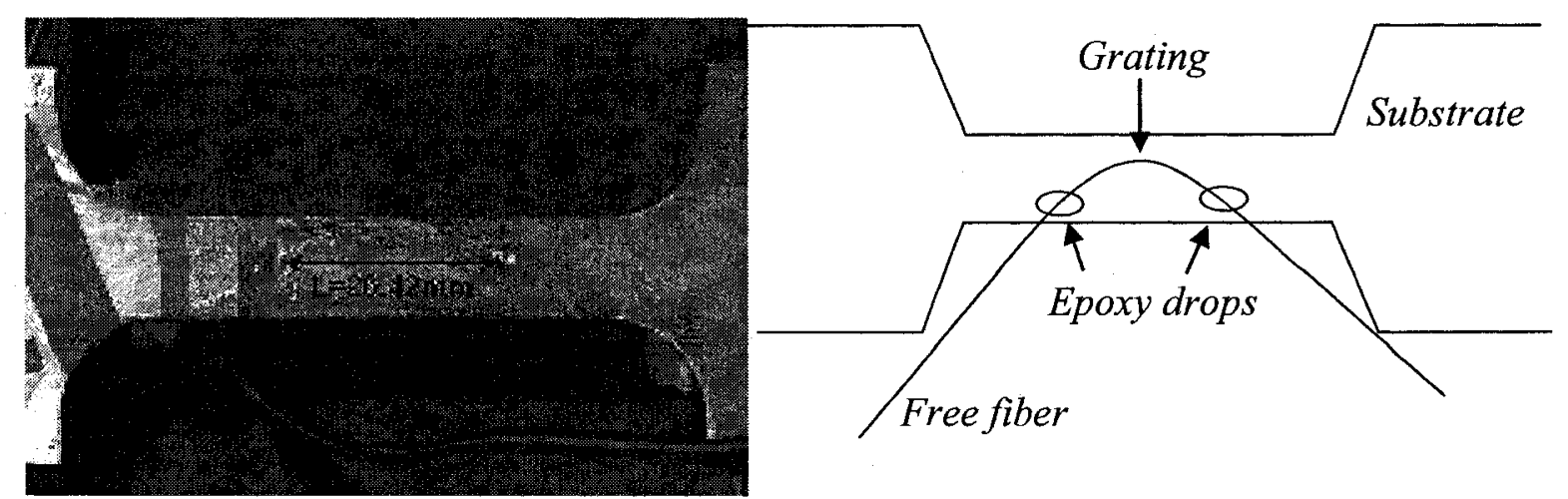

Figure 5.17:TFBG mounting set-up

The aluminium specimen was then axially stretched using a hydraulic pump up to a maximum stress of $28 \mathrm{kN}$ in $2 \mathrm{kN}$ increments. At each increment the transmission 
spectrum was collected using the Micron Optics Optical Sensing Analyzer Si 720 with a resolution of 3pm. The evolution of the transmission spectrum is shown in Figure 5.18. It can be seen that as the specimen is strained the effective arc length increase which in turn increases the curvature radius and decreases the power differential between the two ghost mode peaks. The power difference between the first two ghost modes versus the strain gage reading is plotted in Figure 5.19.

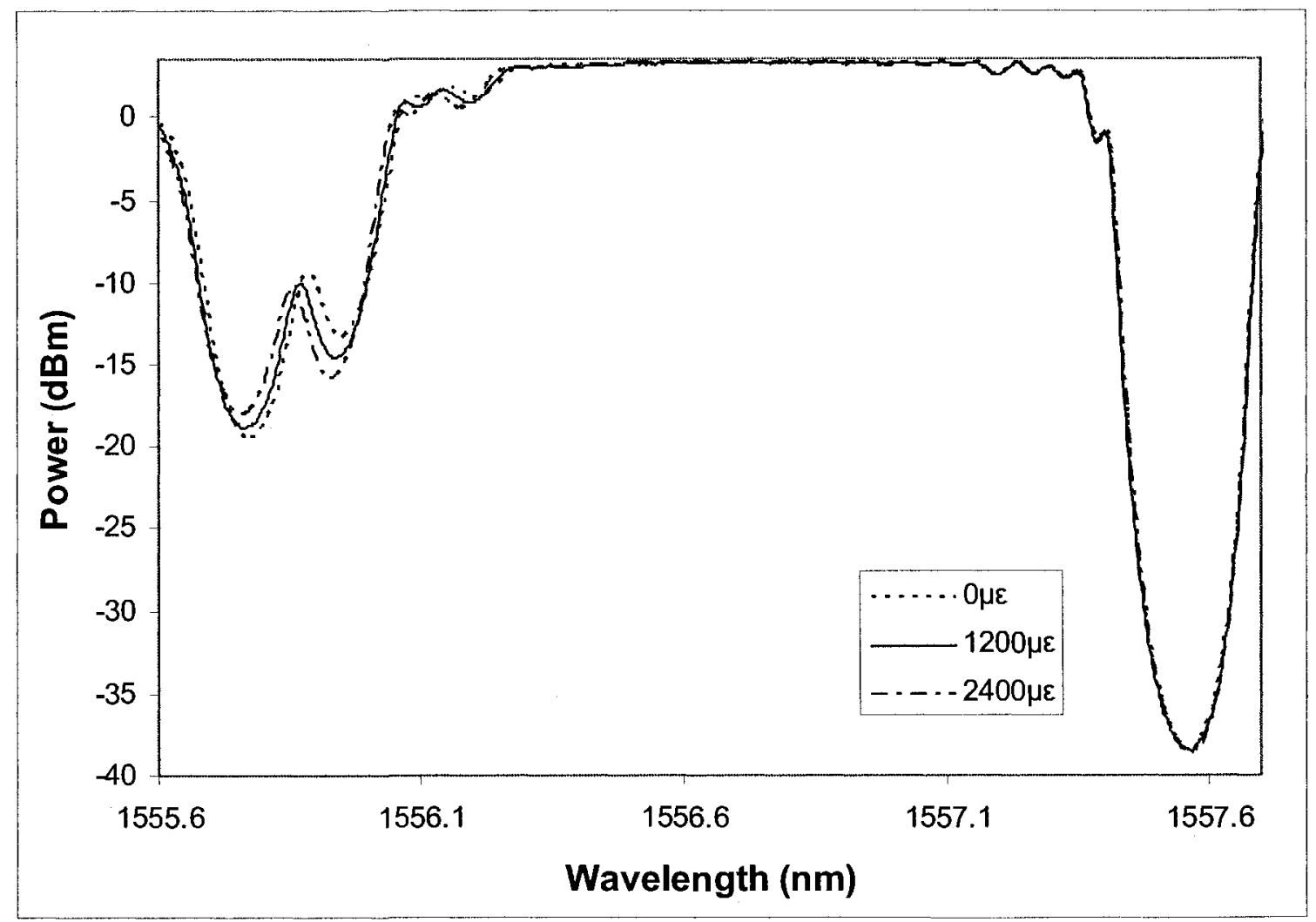

Figure 5.18: Transmission spectrum evolution of a $3^{\circ} \mathrm{TFBG}$ bonded to an aluminum substrate under strain 


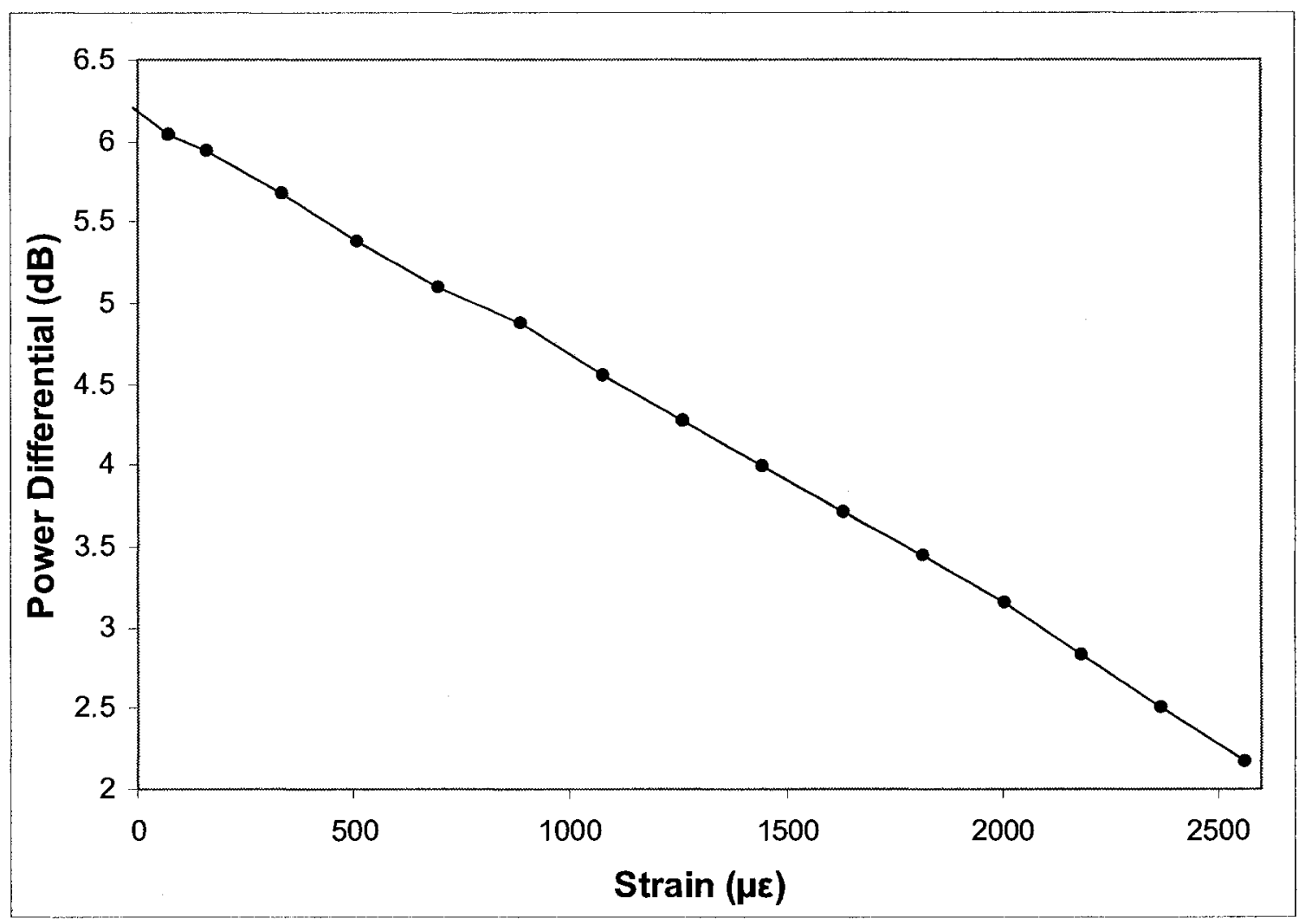

Figure 5.19: Ghost mode power differential vs. strain

As can be seen the response is linear within the range tested. The sensitivity is $1.5 \times 10^{-3} \mathrm{~dB} / \mu \varepsilon$. The test was stopped at $2500 \mu \varepsilon$ to avoid damage to the aluminium substrate however in this configuration the sensor is capable of measuring strains on the order of $10000 \mu \varepsilon(1 \%)$ since, as can be seen in Figure 5.18, the peaks only shifted through a quarter of their range.

\subsubsection{Temperature-Strain Discrimination}

To verify that the strain measurements are indeed temperature independent the aluminium sample was heated to $80^{\circ} \mathrm{C}$ and then cooled down to $-20^{\circ} \mathrm{C}$ with $20^{\circ} \mathrm{C}$ increments inside an Espec SH-241 temperature chamber. The wavelength shift of the 
Bragg resonance was used to monitor temperature and the power differential between the two ghost modes was used to monitor strain. The strain versus temperature plot is shown in Figure 5.20, solid line. Since the aluminium sample was not under stress during the experiment the slope of the response should give the coefficient of thermal expansion of the substrate.

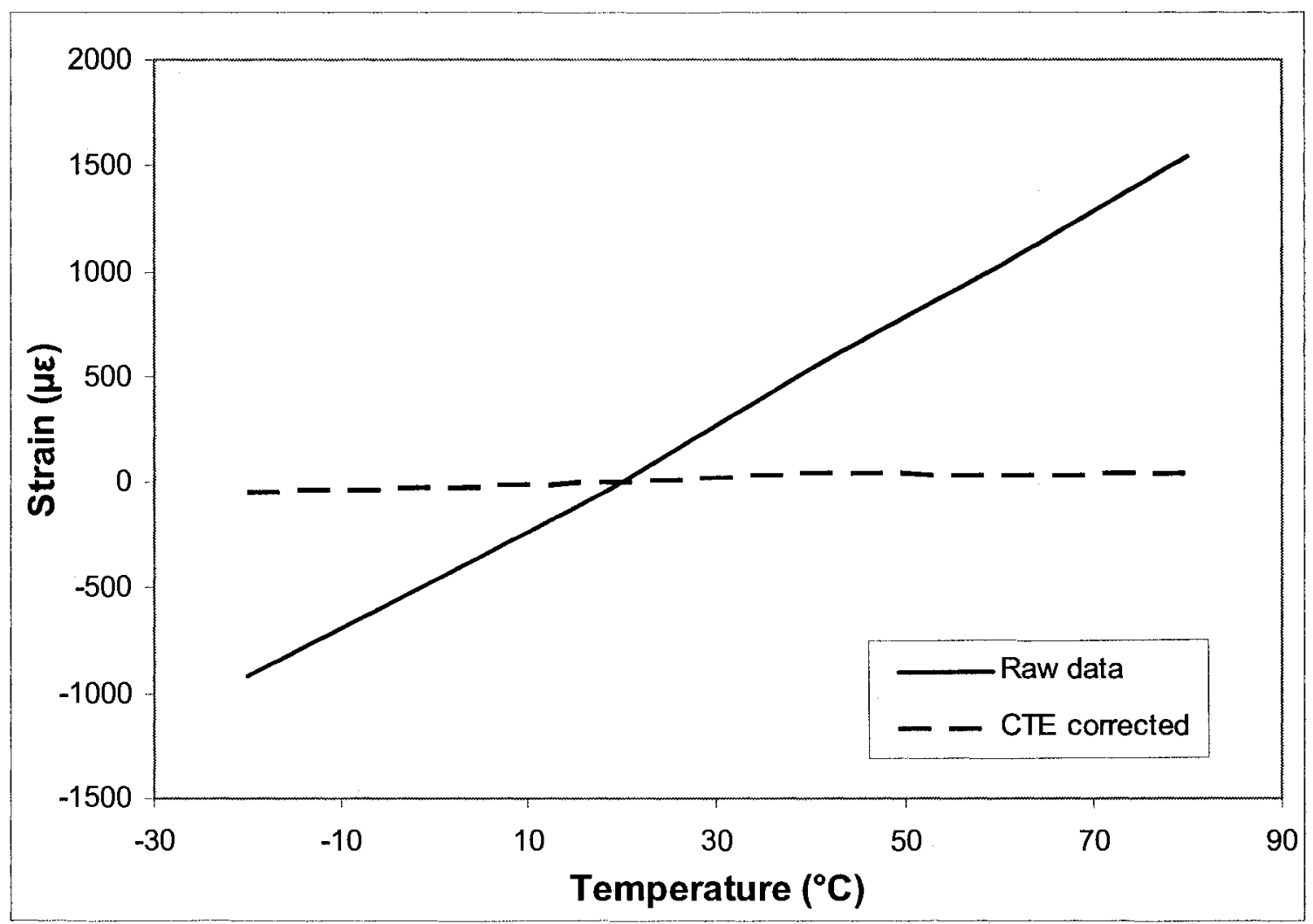

Figure 5.20: Ghost mode power differential vs. temperature

From Figure 5.20 the coefficient of thermal expansion was calculated to be $24.7 \times 10^{-}$ ${ }^{6} / \mathrm{K}$ while the theoretical linear CTE of $6061 \mathrm{~T} 6$ aluminum is $23.6 \times 10^{-6} / \mathrm{K}$, Holt 1996 . The experimental and theoretical values agree reasonably well, the difference is attributed to the nonlinearity of CTE of aluminium for the given test range. 
If the strain response is corrected by the theoretical value of CTE of aluminum a null result is expected. Such temperature-compensated response is shown in Figure 5.20 dashed line. The maximum deviation from zero is $50 \mu \varepsilon$ and is mostly attributed to the nonlinear temperature response of the Bragg and nonlinear CTE of aluminium. With proper packaging such sensor can be used either to measure CTE or if the CTE is known, to monitor strain in the structures where the ambient temperature changes significantly and conventional temperature compensation methods can not be used.

\subsection{Ghost Mode Sensitivity to Bending in Reflection.}

The previous section has showed that TFBGs interrogated in transmission can effectively measure bending because cladding mode coupling is dependent on the internal strains of the fiber. But it is not always convenient to interrogate a fiber in transmission because the signal must be carried to the fiber and back using two different ends of the fibers. For certain applications it is advantageous to minimize the number of fibers and the sensor must be interrogated in reflection. The problem is that normally cladding modes do not appear in reflection. The following section will address that problem. A novel way to recouple some of the low order cladding modes back into the core will be demonstrated and a proof of concept vibration sensor will be tested.

\subsubsection{Ghost Mode Recoupling}

In general, the light from the reflected cladding modes never returns back to the interrogation system because of absorption by the jacket material and bend losses. 
However when the fiber is kept straight such modes can propagate for several centimetres without much loss (especially for low order cladding modes). The trick used here is to use a fiber junction to re-couple some power from these backward propagating cladding modes into the core of the interrogating fiber as shown in Figure 5.21. A fusion splice (very slightly misaligned), or a connectorized junction (fibers terminated in a polished ferrule) have both been tried successfully.

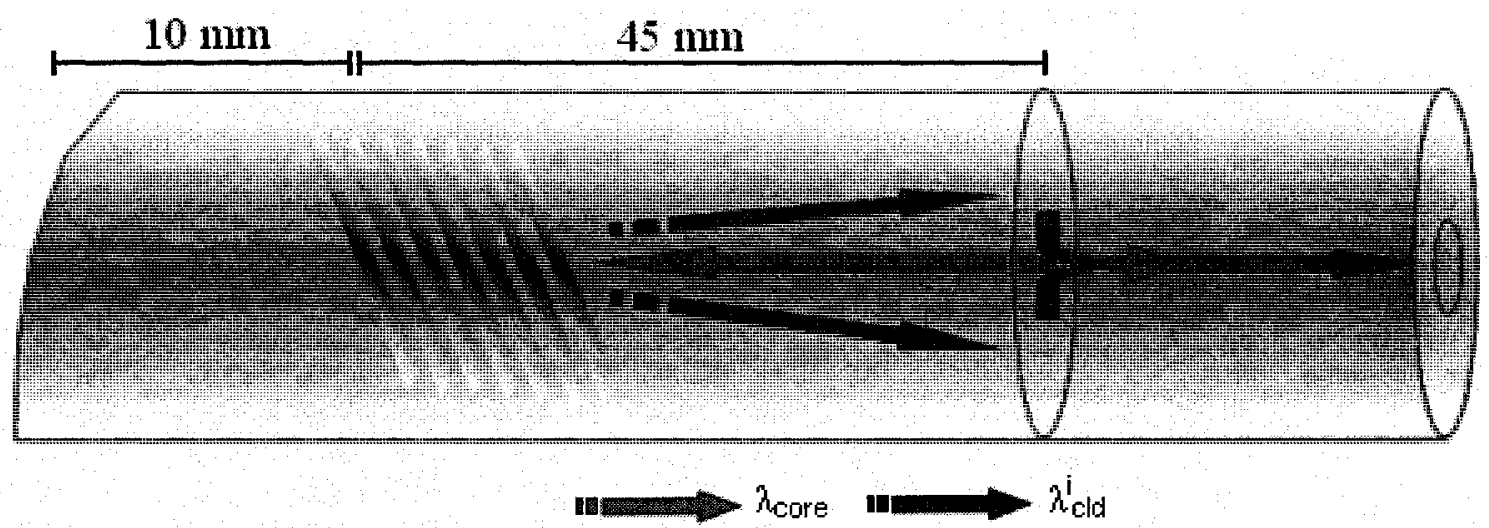

Figure 5.21: Schematic representation of cladding mode recoupling

Once the reflected light is recoupled into the core of the interrogating fiber it behaves exactly like a standard FBG reflection and can propagate as far as needed without additional loss or bend sensitivity. The final reflection spectrum of the tilted FBG then consists of the Bragg peak (longest wavelength) and a few additional peaks on the short wavelength side, corresponding to low order re-coupled cladding modes. Figure 5.22 demonstrates the reflection spectrum of a TFBG with a butt-coupled junction very close to the grating. It can be observed that as the junction is slightly misaligned the coupling to the Bragg mode reflection decreases while the coupling to the ghost mode reflection increases. 


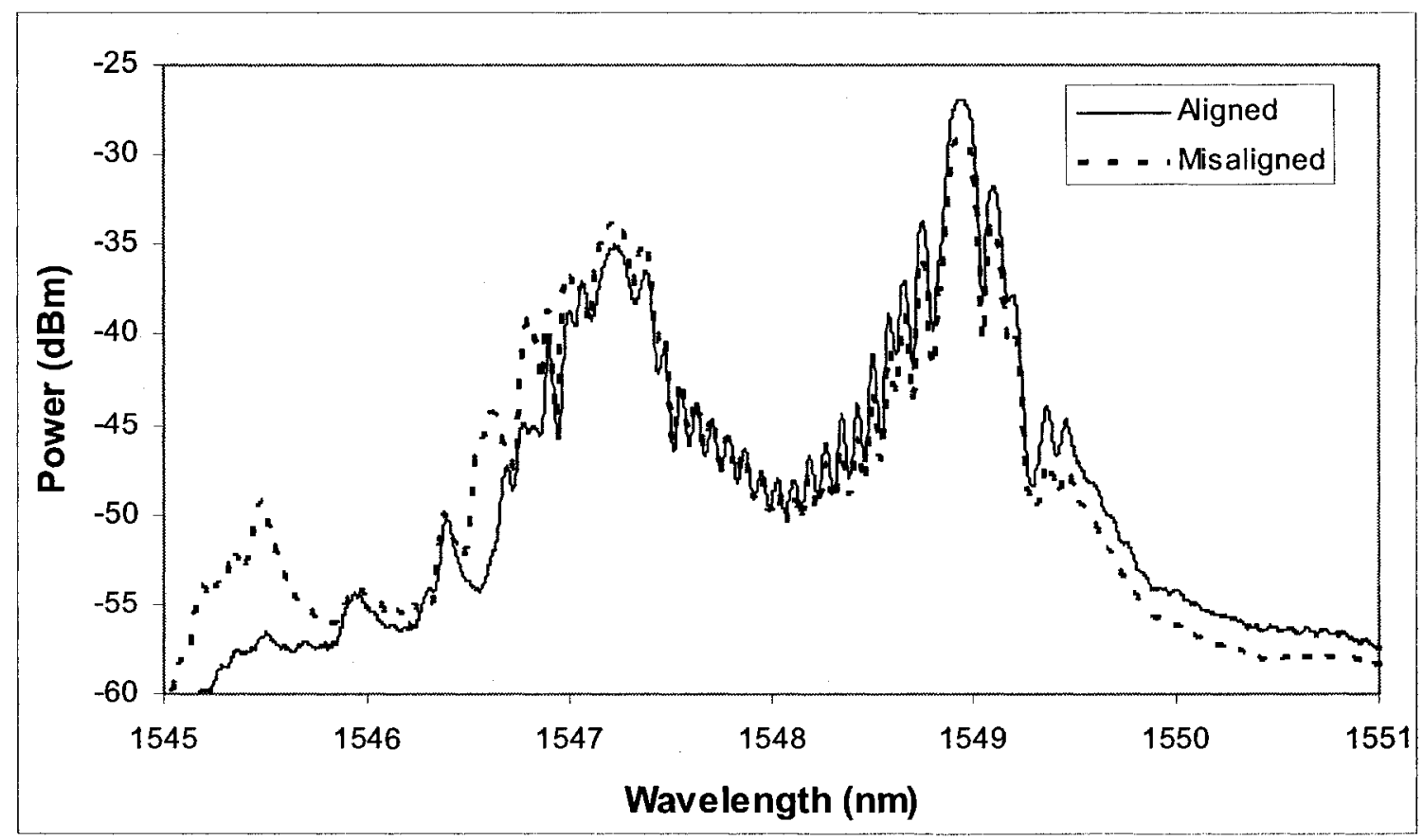

Figure 5.22: Ghost mode recoupling through slight misalignment of the junction

\subsubsection{TFBG Stub Fabrication}

The same $1 \mathrm{~cm}$ tilted gratings as the ones described in section 5.1 were used to fabricate TFBG stubs. This time it is advantageous to have a relatively strong ghost mode because ghost power level is the primary sensing parameter. The Bragg reflection can be weak because only the Bragg wavelength is important. Experimentally $4^{\circ}$ gratings produced the best results. The stubs were spliced and cut as shown in Figure 5.21. A 55 $\mathrm{mm}$-long segment of the fiber containing the grating was cleaved with the grating located at a point $10 \mathrm{~mm}$ away from one end and $35 \mathrm{~mm}$ from another. The short end was cut at an angle to reduce unwanted back reflections while the long end was cleaved perfectly at $90^{\circ}$. Then using a Corning Compact fusion splicer the long end was spliced back to a 
piece of fiber. $62 \mathrm{~mm}$ away from the stub edge, the fiber was clamped on a transverse translation stage and the resultant stub placed $1 \mathrm{~mm}$ above a Peltier heater in order to allow simultaneous deflection and heating tests. The fiber was then deflected $\pm 1.5 \mathrm{~mm}$ with $0.13 \mathrm{~mm}$ step size as shown in Figure 5.23 .

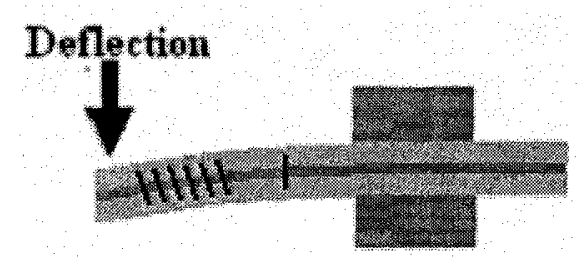

Figure 5.23: Deflection mechanism

The experiment was performed at room temperature and then repeated at 69.8 and $-10.2^{\circ} \mathrm{C}$. The reflection spectrum was saved at each deflection point using an ANDO spectrum analyzer with the resolution set at $0.05 \mathrm{~nm}$.

\subsubsection{Temperature and Bending Results}

Figure 5.24 shows the reflection spectrum at three different temperatures. Temperature causes the shift in wavelength however in this figure the Bragg peaks are purposely shifted back to overlap to show that the ghost power does not vary with temperature. Figure 5.25 shows the reflection at constant temperature and three different deflections. It can be seen that there is no wavelength shift in the spectrum, but there is a significant variation in the ghost mode power thus allowing temperature independent bend measurements. 


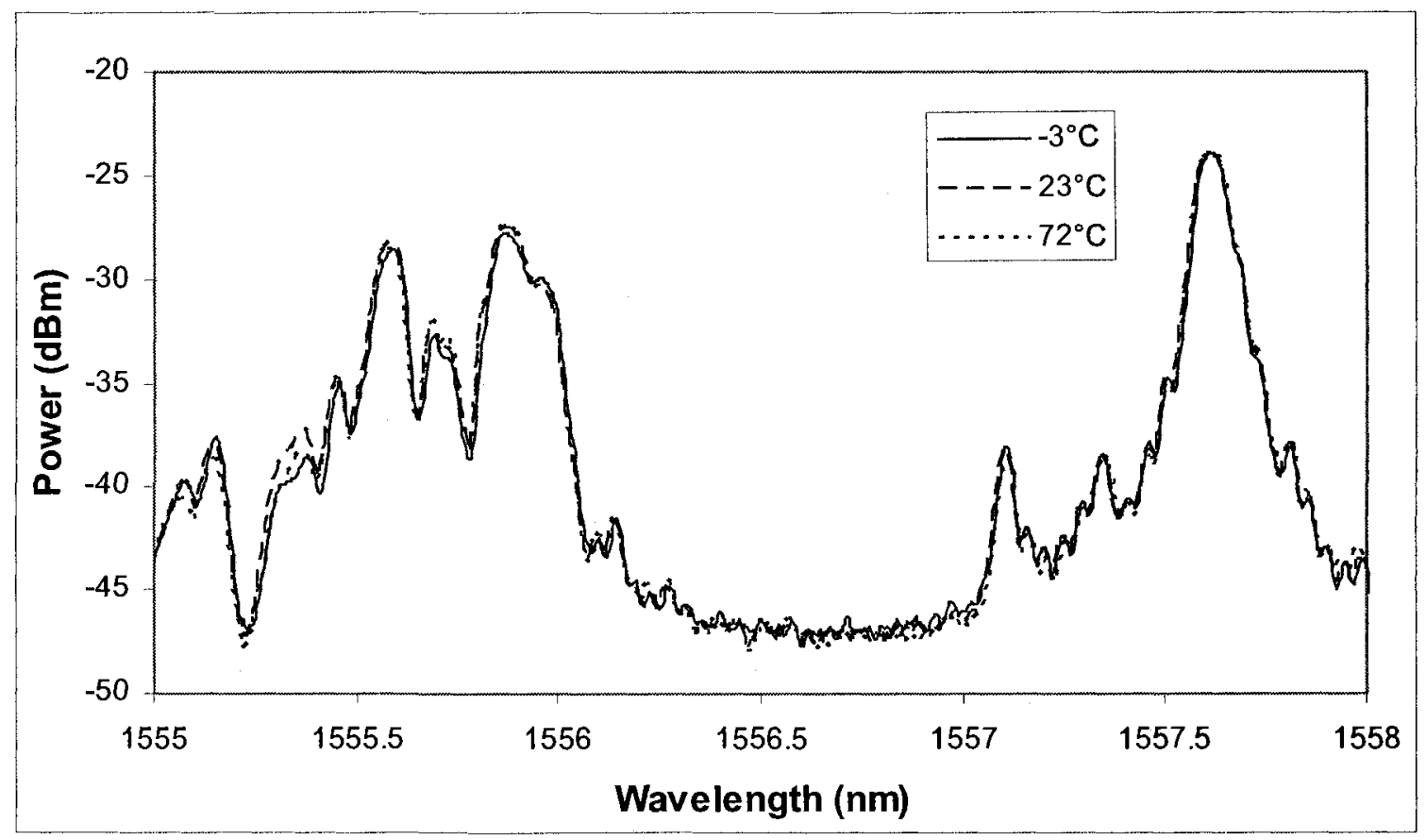

Figure 5.24: Reflection spectrum of a TFBG stub at three different temperatures

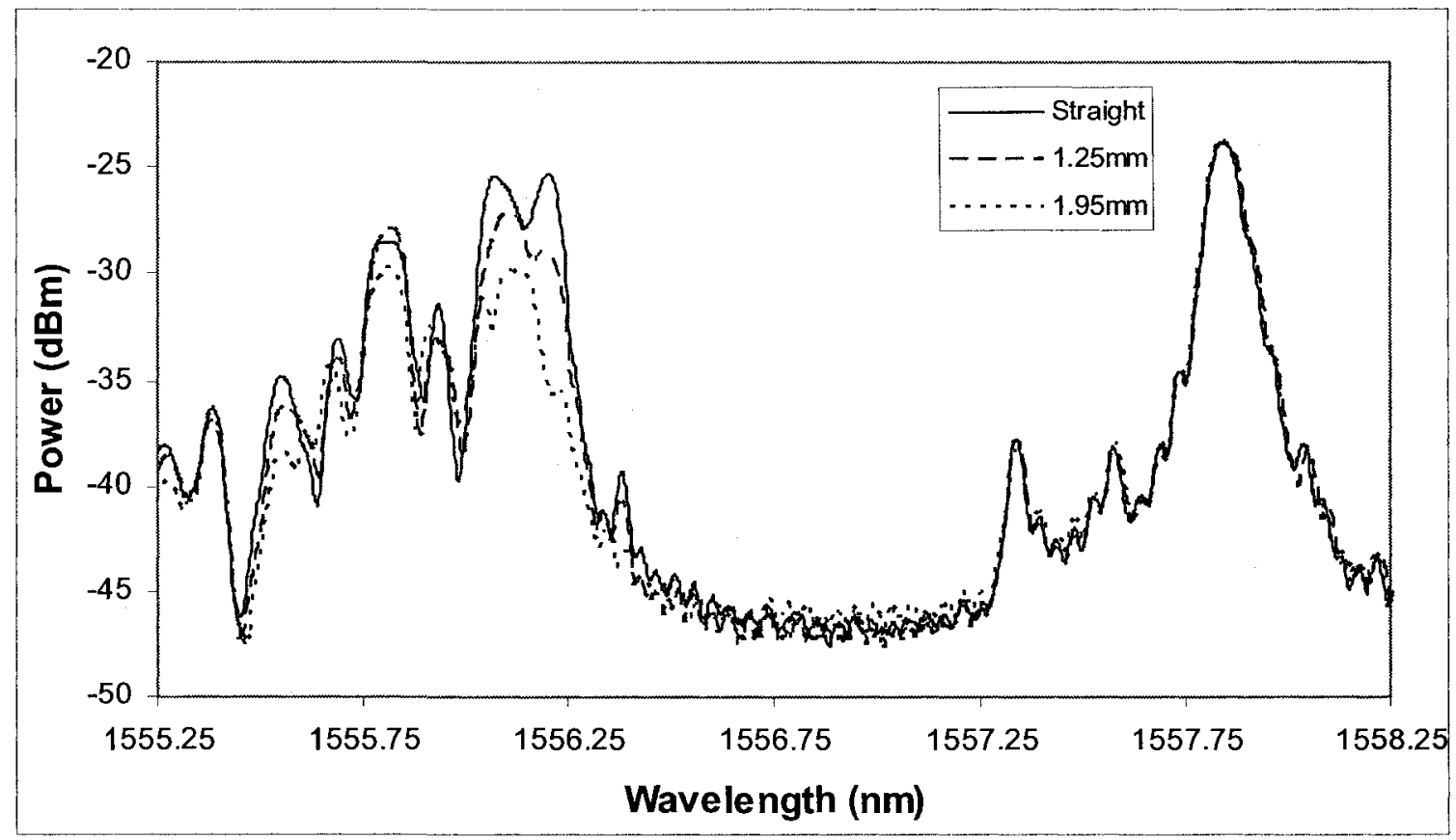

Figure 5.25: Reflection spectrum of a TFBG at room temperature and three different deflections 
Figure 5.26 shows the power variation inside a $128 \mathrm{pm}$ wide, wavelength window located $1.881 \mathrm{~nm}$ away from the Bragg peak, as a function of deflection. It can be noticed that the slope of the sensitivities is indeed independent of temperature; however there is an absolute power variation for positive deflection. This discrepancy is most likely due to lack of control of the fiber orientation during bending and asymmetries which arise due to the writing of the grating from one side only.

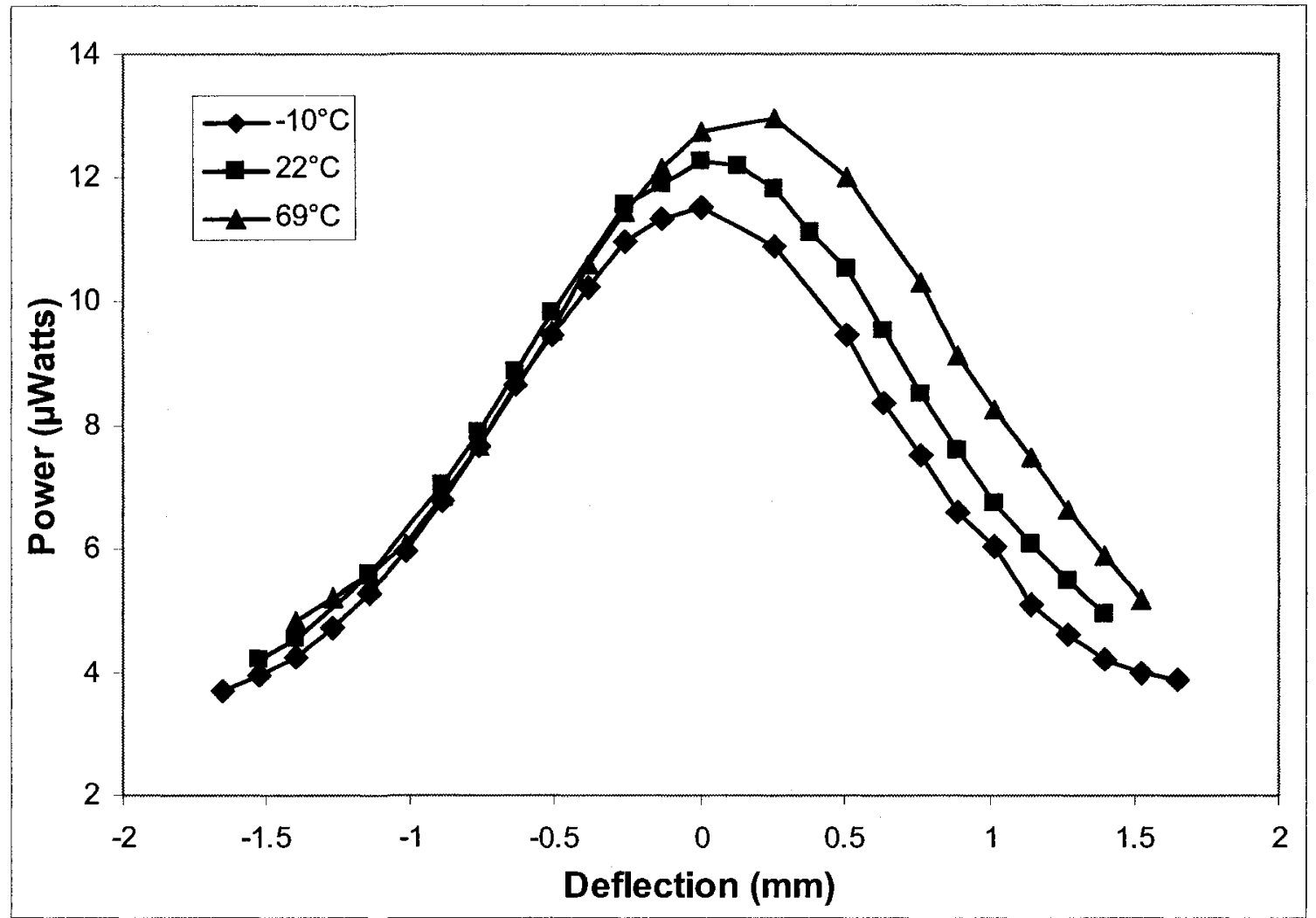

Figure 5.26: Power versus deflection at three different temperatures 


\subsubsection{Proof of Concept Vibration Sensor Demonstration.}

The bending experiments can be directly extended to vibration by applying the deflection dynamically and monitoring the grating spectrum in real time. Such sensor could be useful for shape monitoring and frequency based SHM methods.

For the proof of concept testing, a strong 3 degree grating with the transmission spectrum shown in Figure 5.27 was selected.

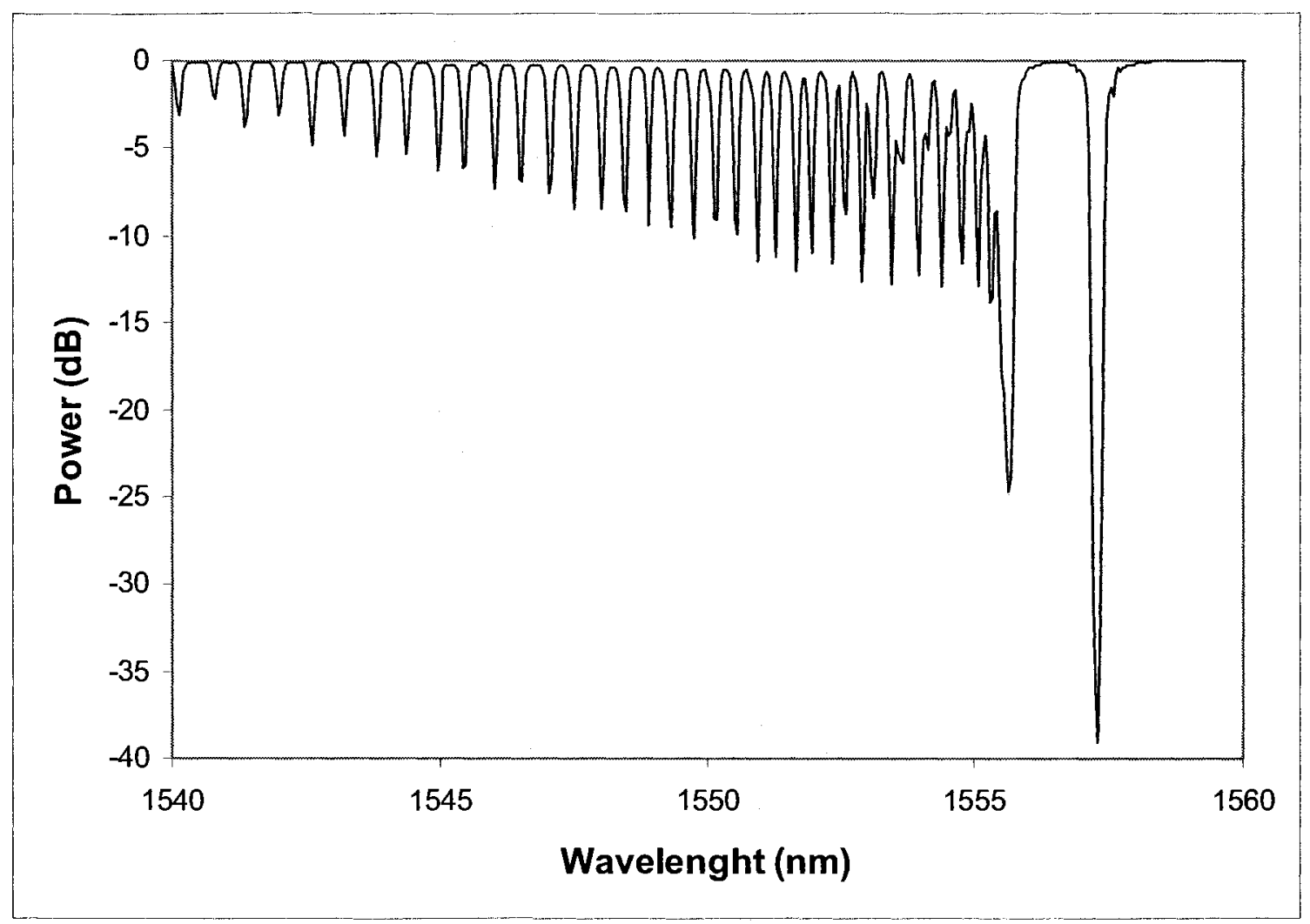

Figure 5.27: $3^{\circ}$ tilted grating transmission spectrum

A bad splice was then made close to the grating as discussed in section 5.3 in order to recouple the ghost mode when the grating is interrogated in reflection. The reflection spectrum of the grating with the bad splice is shown in Figure 5.28. It can be observed that quite a lot of cladding modes got recoupled. Since most of them are significantly 
weaker than the Ghost and would probably introduce noise in the measurements the grating has been recoated with a conventional UV-cured resin, Desotech, 2007, which causes the higher order cladding modes to leak out by raising the external refractive index. The recoat provides protection as well.

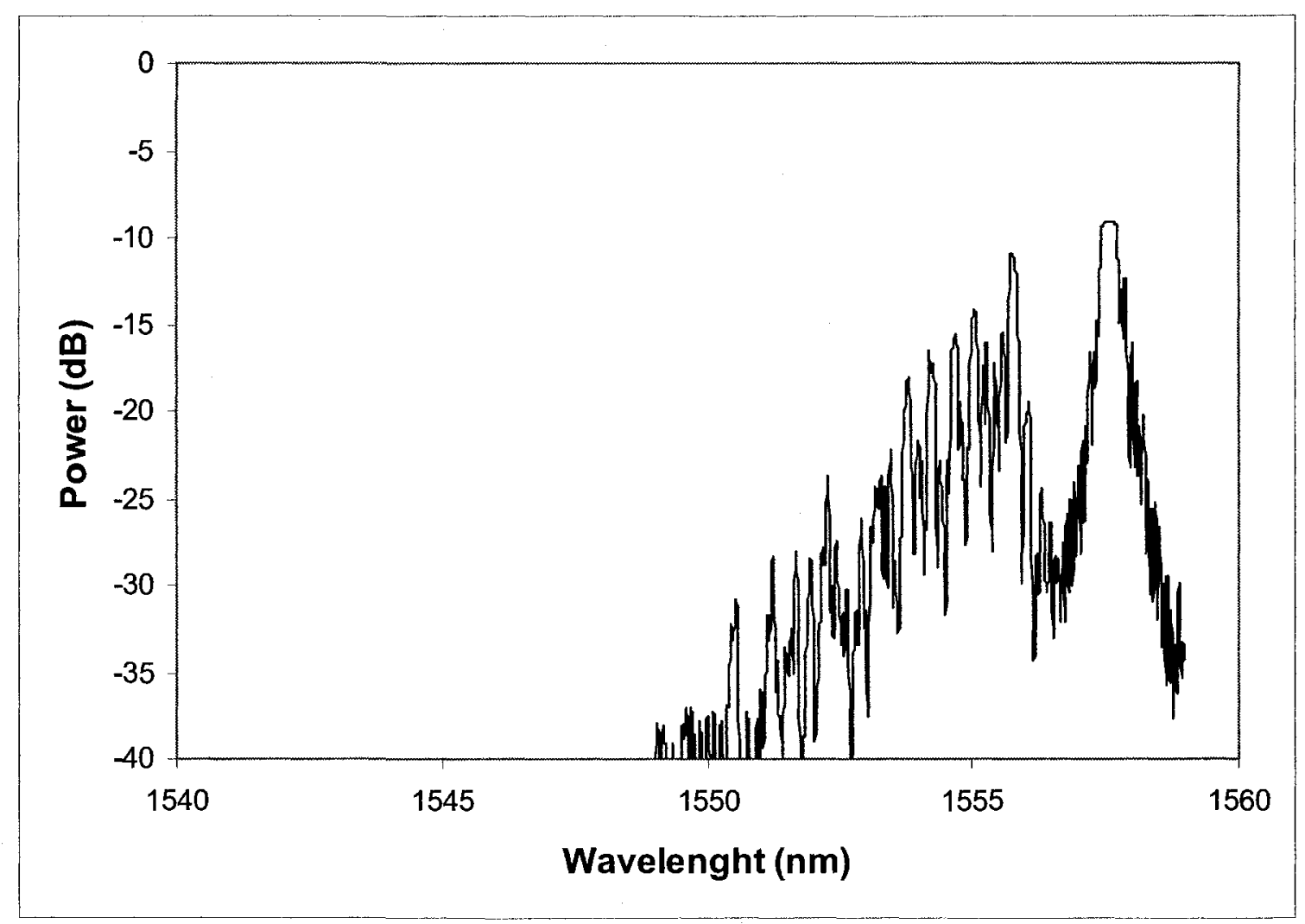

Figure 5.28: Reflection spectrum with the bad splice

As shown in Figure 5.29 after recoat a significant amount of cladding modes got suppressed leaving mostly just the ghost mode and the Bragg. 


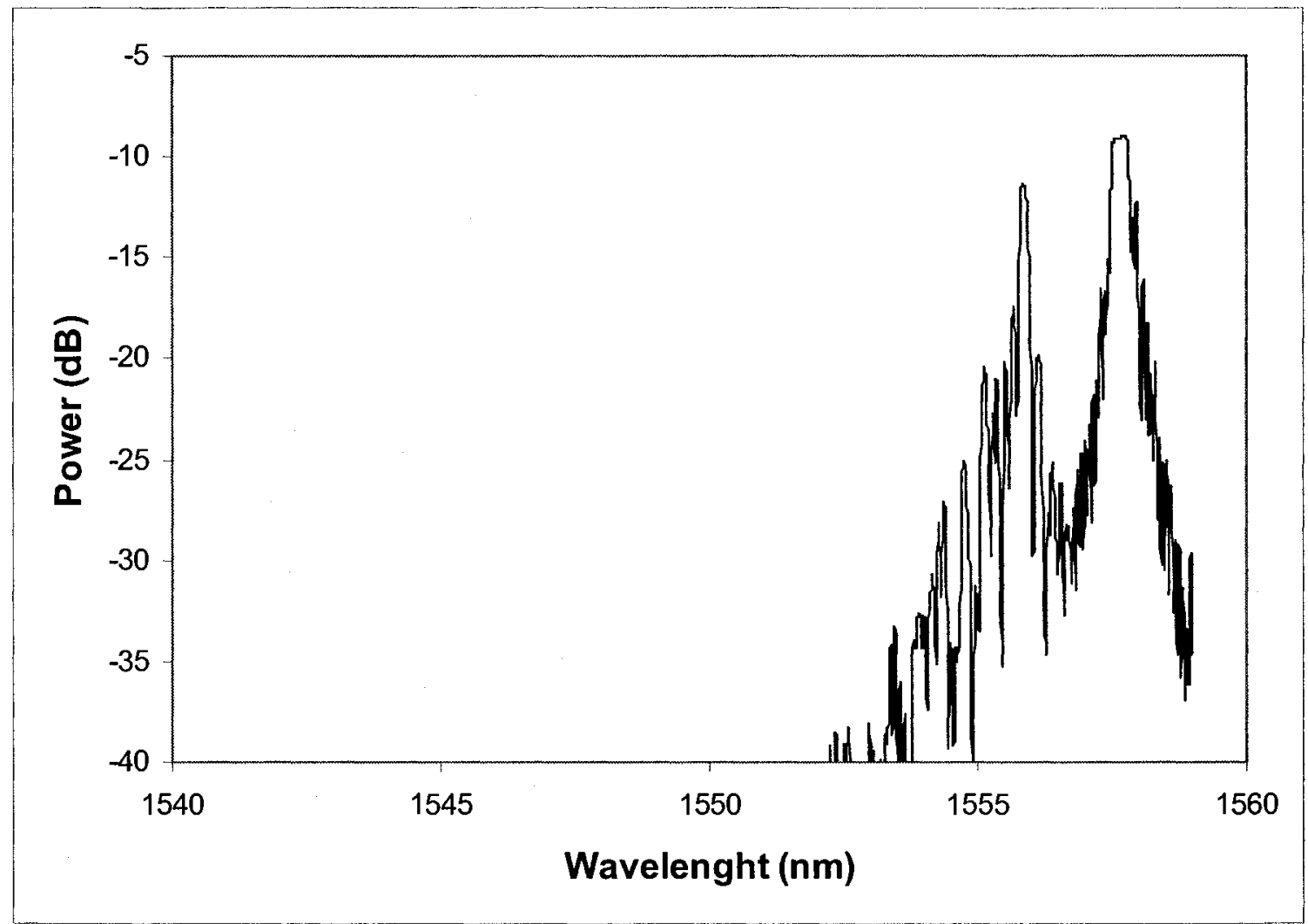

Figure 5.29: Recoated TFBG reflection spectrum

To apply dynamic deflection with a known frequency the set up shown in Figure 5.30 has been used. The shaker was implemented using a speaker cone. The signal driving the speaker was generated using a Test Tone Generator 4.21 software and standard computer

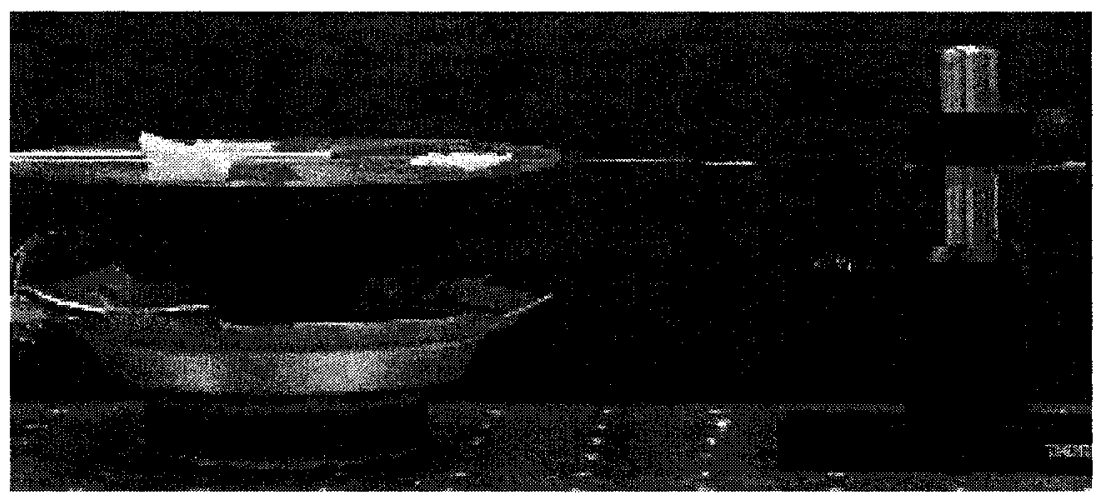

Figure 5.30: Dynamic deflection testing set up 
sound card. The grating was illuminated with a broadband light source and interrogated by and ANDO spectrum analyzer. To measure the dynamic response the analyzer was set to span zero centered at the ghost peak with the data acquisition rate set at $1 \mathrm{kHz}$. The speaker was then set to vibrate with the frequency of $39 \mathrm{~Hz}$ and the power was recorded for a duration of 1 second. The same procedure was then repeated for the Bragg. The vibration response of both peaks is shown in Figure 5.31.

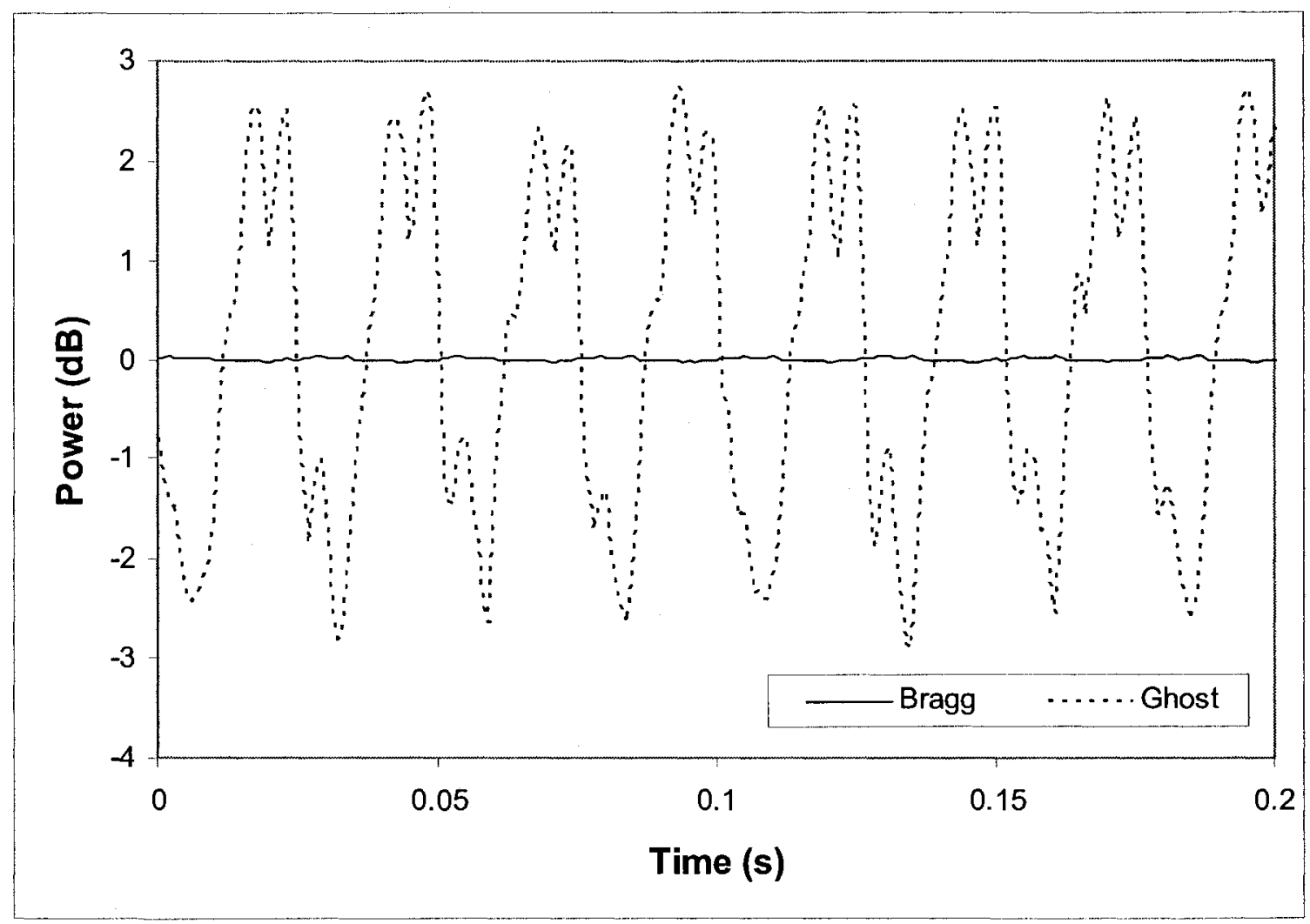

Figure 5.31: Vibration responce Bragg and Ghost modes

It is quite clear that the Ghost is very sensitive to bending while the Bragg is not. However it is impractical to use a spectrum analyzer to interrogate such system because of the limited sampling rate and the associated costs. A better design is to use a power 
detector at the output and measure all of the returning power. Since the Bragg is not sensitive to bending only the fluctuations in the ghost should be picked up by the detector and a voltage signal similar to the response in Figure 5.31 should be obtained. However it should be noted that the constant power reflected from the Bragg will reduce the dynamic range of the system.

For testing purposes a high speed, biased ThorLabs D400FC, InGaAs photo-detector has been used. The bandwidth of such detector is $1 \mathrm{GHz}$ and it has the sensitivity of 0.95 $\mathrm{A} / \mathrm{W}$ at $1550 \mathrm{~nm}$. The signal can be read by an oscilloscope connected across a load resistor. For the fastest response a $50 \Omega$ resistor is recommended but since in our case the frequency was not to exceed $100 \mathrm{~Hz}$ due to the limitations of the shaker design a larger $9.6 \mathrm{k} \Omega$ load resistor has been used to amplify the voltage swing. Initial tests showed that the signal power levels of the sensor illuminated by the broadband source were on the order of $2 \mu \mathrm{W}$ which is too low to be detected. Therefore a more powerful multi-line laser source has been used. The reflection spectrum of the grating illuminated by the multi-line laser source is shown in Figure 5.32. When illuminated by the multi-line laser source the signal power went up to $50 \mu \mathrm{W}$ and was detectable by the photo diode. The signal recorded by an oscilloscope in response to a $39 \mathrm{~Hz}$ vibration is shown in Figure 5.33. The response is quite noisy due to the noise of the detector and the small wavelength shifts which turn into large power fluctuations when a multi-line laser line is not aligned with the peaks. One way to optimize it is to use an unbiased, amplified photo-detector in conjunction with a broadband source which would reduce the noise. 


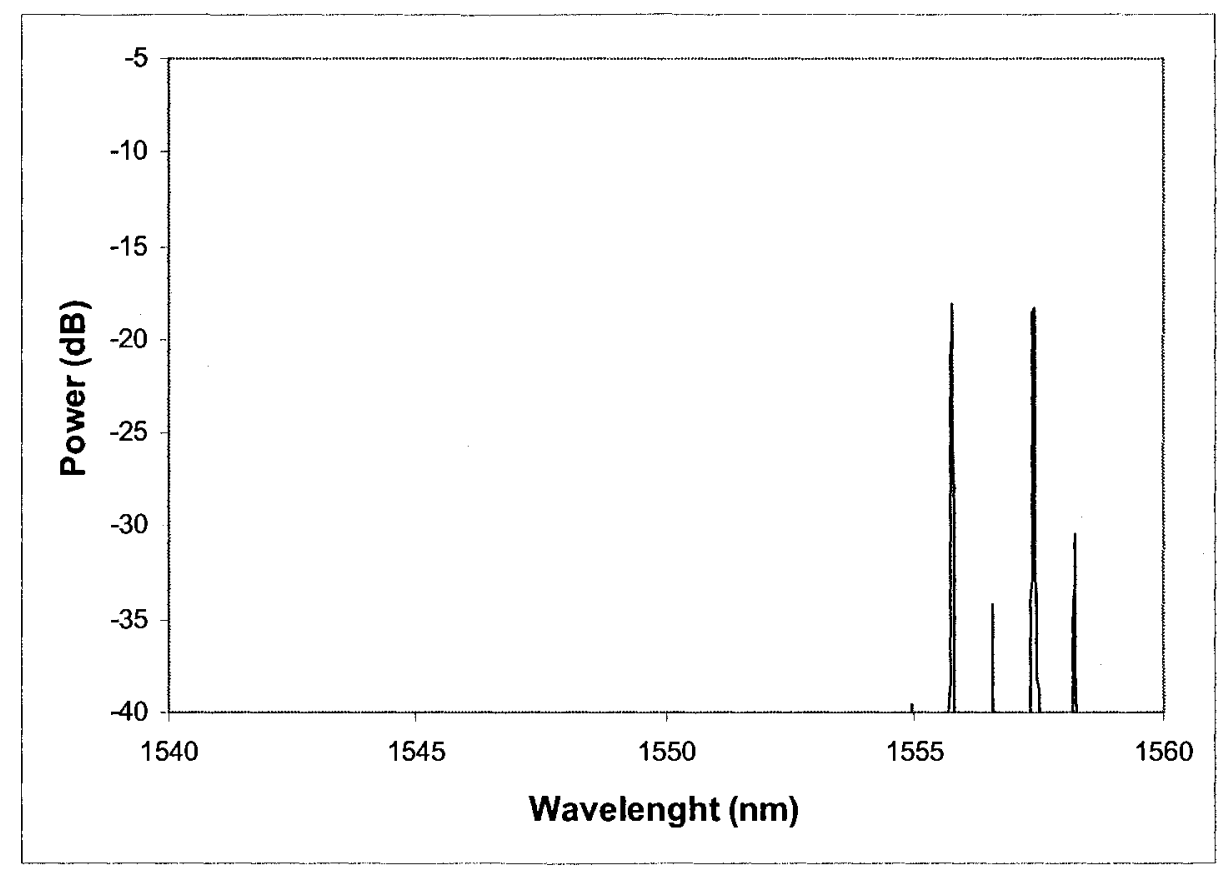

Figure 5.32: Grating reflection spectrum illuminated by the multi line laser source

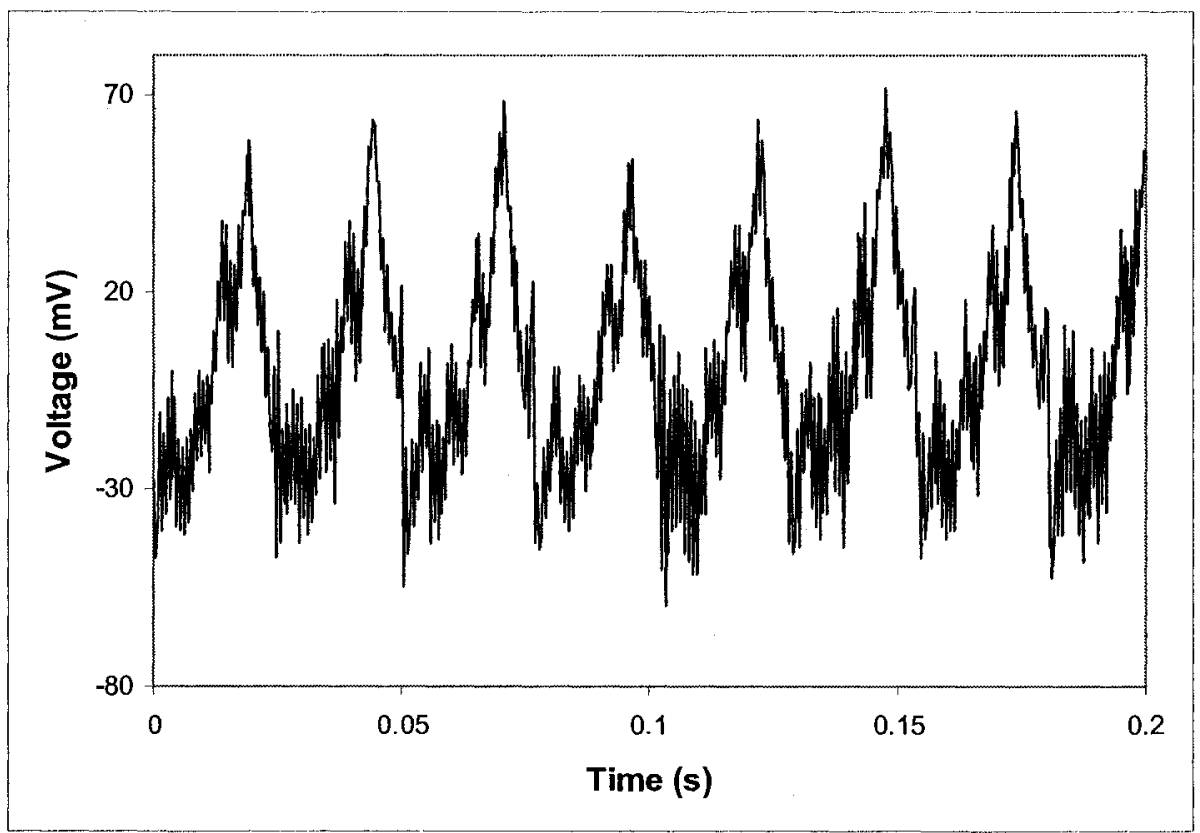

Figure 5.33: TFBG electrical response to vibration 


\subsection{Summary}

This chapter presented the use of TFBGs for temperature, bend, strain and vibration sensing. The experiments have been broken down by the interrogation method of the sensor; into the ones done in transmission and in reflection. In transmission the bend and temperature sensitivity as well as the bending orientation have been analyzed in depth. A temperature compensated strain gauge design has been proposed. This work has been accepted as an oral presentation at an international conference Ivanov \& Albert, 2007a. In reflection a novel way of recoupling reflected cladding modes back into the core has been demonstrated and an inexpensive vibration stub sensor tested. The proceedings of this work led to a poster presentation Ivanov \& Albert, 2007b and a journal publication Guo et al. 2008. The temperature, strain and bending sensitivity of TFBG have also been included in a patent "Tilted grating sensor", international filing number: PCT/CA2006/001749. 


\section{Chapter 6: Conclusions and future work}

\subsection{Conclusions}

As indicated in the literature review there are many requirements for the sensors for SHM systems and many of them depend on the specific application. However, there are characteristics which are common to most of them. Usually multiple parameters need to be measured with a single sensor that is capable to discriminate between them. The operational conditions are quite often very harsh: sensors must withstand corrosive environments such as sea water, extreme temperatures and electromagnetic interference. Furthermore, $\mathrm{MHz}$ acquisition speeds are required in certain cases and at the same time te cost must be kept low.

Intrinsically, fiber optic sensors are well suited to meet the above requirements. However most designs either lack the temperature discrimination or are very difficult to multiplex and are consequently costly to interrogate. Wavelength division multiplexing is one of the easiest schemes and therefore dual grating temperature and strain discrimination has been identified as one of the most attractive solutions. In the first part of this thesis a detailed investigation of the temperature and strain sensitivity of FBGs with different coatings, configurations and wavelengths within the C-band has been carried out. The results agree well with theoretical predictions and previously reported 
results. Acrylic coated gratings have been shown to have a limited temperature operational range and low strain transfer capability. Polyamide gratings on the other hand provide good strain transfer and withstand temperature range suitable for most applications. However the temperature and strain discrimination is not possible with FBGs with closely spaced wavelengths.

Tilted FBGs have also been studied in great detail. For the first time, the orientation dependent bending sensitivity has been addressed for all of the cladding modes. A temperature independent bend sensor based on the first cladding mode only has also been demonstrated. Finally a novel method of coupling cladding modes back into the core and interrogating tilted FBGs in reflection has been developed, and proof of concept vibration sensor interrogated by a simple photodiode has been successfully implemented.

\subsection{Recommendations for Future Work}

For continuing the work of developing a multi-parameter sensor for SHM that was started in this thesis, there are several areas which require more attention.

Dual gratings did not show much potential in temperature and strain discrimination unless certain limitations can be overcome, such as finding ways to have co-located FBG with large wavelength separation.

On the subject of tilted FBGs, it has been demonstrated that temperature independent bending and vibration measurements are possible. However the multiplexing issue has only been addressed briefly. More work is required with various coating in order to determine if it is indeed possible to strip away unwanted cladding modes and at the same time be able to multiplex many gratings at different wavelengths on the same fiber line. 
The polarization dependence of the TFBG sensor must also be further examined. In this work non-polarised light has been used in most instances, but if the sensor was indeed to be integrated in a compact system it is quite possible that inexpensive laser diodes with uncontrolled polarization would be used. In this case the results might depend on polarization because gratings are intrinsically birefringent due to the side writing technique and the tilt of the grating planes.

Higher frequency vibration testing is also required. Perhaps a more application specific approach must be chosen such that frequency and amplitude requirements are better defined. Better electronics and photo-detectors are also required. As has been demonstrated experimentally the optical power changes are typically very small and therefore the electrical signal must be amplified significantly, but as the modulation frequency gets higher the response time of the detector also becomes very important.

Finally a solid numerical model needs to be developed in order to better understand the mechanisms behind the cladding mode recoupling due to bending and vibration. The model needs to take into account both the lossy cladding modes and the bends of the fiber simultaneously. 


\section{Chapter 7: Publications and Patents}

T. Guo, A. Ivanov, C. Chen, and J. Albert "Temperature-independent tilted fiber grating vibration sensor based on cladding-core recoupling" Optics letters, accepted for publication, 2008.

A. Ivanov and J. Albert, "Bent tilted fiber Bragg gratings for temperature-independent vibration sensing in vehicles", Proceedings of Photonics in the Trasportation Industry: Auto to Aerospace, SPIE, 6758-2, $2007 \mathrm{a}$.

A. Ivanov and J. Albert, "Tilted fiber Bragg gratings stubs for vibration and bend sensing", Proceedings of the OSA Topical Meetings on Bragg Gratings, Photosensitivity and Poling in Glass Waveguides, paper JWA49, 2007b.

N. Mrad, A. Ivanov, J. Albert and G. Xao, "Characterization of fibre Bragg gratings for temperature and strain sensing", proceedings of Structural Health Monitoring and Nondestructive Evaluation SPIE, Vol. 6529, pp. 65290T,2007.

J. Albert, C. Chen, Y. Shevchenko and A. Ivanov, "Tilted grating sensor", International application No. : PCT/CA2006/001749, International filing date : 25 October 2006 


\section{Chapter 8: References}

M. R. Alam and A.S.J. Swamidas, "A non-destructive crack detection method for steel jacket offshore platforms based on global and local responses", Canadian Journal of Civil Engineering, Vol. 29, pp. $85-97,2002$.

J. Albert, C. Chen, Y. Shevchenko and A. Ivanov, "Tilted grating sensor", International application No. : PCT/CA2006/001749, International filing date : 25 October 2006

S. Baek, Y. Jeong, and B. Lee, "Characteristics of short-period blazed fiber Bragg gratings for use as macro-bending sensors," Applied. Optics, Vol. 41, pp. 631-636, 2002.

$\mathrm{X}$. Bao and Liang Chen, "Development of the distributed Brillouin sensor for health monitoring of civil structures" Proceedings of Optical Waveguide Sensing and Imaging conference, pp. 101, 2006

K. Bennett, J. Koh, J. Coon, C.K. Chien, A. Artuso, X. Chen, D. Nolan, M.-J. Li, "Specialty fibers for fiber optic sensor application", Proceedings of Fiber Optic Sensors and Applications V, SPIE, 67700M$1,2007$.

U. Ben-Simon, I. Kressel, Y. Botsev, A.K. Green, G. Ghilai, N. Gorbatov, M. Tur and S. Gali, "Residual strain measurement in bonded composite repairs for aging aircraft by embedded fiber Bragg grating sensors", Proceedings of Third European Workshop on Optical Fibre Sensors, SPIE, Vol. 6619, $661944,2007$.

D.C. Betz, G. Thursby, B. Culshaw and W.J. Staszewski, “Acousto-ultrasonic sensing using fiber Bragg gratings", Proceedings of Smart Materials and Structures,SPIE, Vol. 12, pp.122-128, 2003.

U. L. Block, V. Dangui, M. J. F. Digonnet and M. M. Fejer, "Origin of Apparent Resonance Mode Splitting in Bent Long-Period Fiber Gratings," Journal of the Lightwave Technology, Vol. 24, pp. 1027-1034, 2006.

G. P. Brady, K. Kalli, D. J. Webb, D. A. Jackson, L. Zhangt and I. Bennion, "Recent developments in optical fibre sensing using fibre Bragg gratings", Proceedings of Fiber Optic and Laser Sensors, SPIE, Vol. 2839, pp. 8-19, 1996.

S. Calvert and J. Mooney, "Bridge structural health monitoring system using fiber grating sensors: development and preparation for a permanent installation" Proceedings of Sensors and Smart Structures Technologies for Civil, Mechanical, and Aerospace Systems, SPIE, Vol. 5391, pp. 61-71, 2004. 
P. Capoluongo, C. Ambrosino, S. Campopiano, A. Cutolo, A. Cusano, M. Giordano, I. Bovio and L. Lecce, "Fiber Bragg Grating Sensors for High Frequency Damage Detection Applications", 1st International Conference on Sensing Technology, pp. 371-376, 2005.

P. Capoluongo, C. Ambrosino, S. Campopiano, A. Cutolo, M. Giordano, I. Bovioc, L. Lecce, A. Cusano, "Modal analysis and damage detection by Fiber Bragg grating sensors", Sensors and actuators $A$, Vol. 133, pp. 415-424, 2007

E.P. Carden and P. Fanning, "Vibration Based Condition Monitoring: A Review", Structural Health Monitoring,, Vol 3(4), pp. 355-377, 2004

C. Caucheteur, F. Lhomm'e, F. Maakaroun, K. Chah, M. Blondel and P. M'egret, "Simultaneous strain and temperature sensor using superimposed tilted Bragg gratings", Proceedings Symposium IEEE/LEOS Benelux Chapter, pp.219-222, 2004.

C. Caucheteur, K. Chah, F. Lhommé, M. Blondel and P. Mégret, "Simultaneous bend and temperature sensor using tilted FBG", Proceedings of the 17th International Conference on Optical Fibre Sensors, SPIE, Vol. 5855, pp. 707-710, 2005.

C. Chen and J. Albert "Strain-optic coefficients of individual cladding modes of singlemode fibre: theory and experiment" Electronics letters, Vol. 42(18), pp. 1027-1028, 2006.

H.L. Chen, C.C. Spyrakos, and G. Venkatesh, "Evaluating structural deterioration by dynamic response". Journal of Structural Engineering, Vol. 121(8), pp. 1197-1204, 1995.

L.K. Cheng, "High-speed Dense Channel Fiber Bragg Grating Sensor Array For Structural Health Monitoring" Proceedings of the 2005 International Conference on MEMS, NANO and Smart Systems, pp. 820-821, 2005.

A.A. Clifford, Multivariate Error Analysis, London: Applied Science Publishers LTD, 1973.

Desotech Inc. Desolite 950-200 data sheet issue 07/01

S.W. Doebling, C.R. Farrar, M. B. Prime and D.W. Shevitz, "Damage identification and health monitoring of structural and mechanical systems from changes in their vibration characteristics: A literature review," Los Alamos National Lab, NM, United States, Tech. Rep. LA--13070-MS, 1996.

EDL inc, "Typical Linear coefficient of expansion for common Plastics", online database http://www.edl-inc.com/Plastic\%20expansion\%20rates.htm, September 2006

Engineers handbook, "Plastics thermal expansion coefficients", online database http://www.engineershandbook.com/Tables/plasticthermalexp.htm, September 2006,

T. Erdogan, “Fiber Grating Spectra”, Journal of Lightwave Technology, Vol. 15(8), 1997

A. Fender, E.J. Rigg, R.R.J. Maier, W.N. MacPherson, J.S. Barton, A.J. Moore, J.D.C. Jones, D. Zhao, L. Zhang, I. Bennion, S. McCulloch, and B.J.S. Jones "Dynamic two-axis curvature measurement using multicore fiber Bragg gratings interrogated by arrayed waveguide gratings", Applied Optics, Vol. 45(36), pp. 9041-9048, 2006. 
N.E. Fisher, D.J. Webb, C.N. Pannell, D.A. Jackson, L.R. Gavrilov, J.W. Hand, L. Zhang and I. Bennion, "Ultrasonic field and temperature sensor based on short in-fiber Bragg Gratings" Electronics Letters, Vol. 34, pp.1139-1140, 1998.

G.M.H. Flockhart, R.R.J. Maier, J.S. Barton, W.N.MacPherson, J.D.C. Jones, K.E. Chisholm, L. Zhang, I. Bennion, "Quadratic behaviour of fber Bragg grating temperature coefficients", Applied Optics, Vol. 43, pp. 2744-2751, 2004.

M.Y. Fu, "The Improvement of the Backward Cladding-Core Coupling in a Tilted Fiber Grating by Two Acoustic Flexural Waves", Journal of Lightwave Technology, Vol. 25(9), pp. 2751-2756, 2007.

D.P. Garg, M.A. Zikry, G.L. Anderson and D. Stepp, "Health Monitoring and Reliability of Adaptive Heterogeneous Structures", Structural Health Monitoring, Vol 1(1), pp. 23-38, 2002.

G.Ghosh, "Temperature dispersion of refractive indexes in some silicate fiber glasses" Photonics Technology Letters, Vol.6(3), pp. 431-433, 1994.

T. Guo, A. Ivanov, C. Chen, and J. Albert "Temperature-independent tilted fiber grating vibration sensor based on cladding-core recoupling" Optics letters, accepted for publication, 2008.

T. Graver, D. Inaudi and J. Doornink, "Growing Market Acceptance for Fiber-Optic Solutions in Civil Structures" Proceedings of Fiber Optic Sensor Technology and Applications III, SPIE, Vol. 5589, pp. 44-55, 2004.

B.O.Guan, H.Y.Tam, S.L. Ho, W.H. Chung and X.Y.Dong, "Simultaneous strain and temperature measurement using a single fibre Bragg grating", Electronics Letters, Vol. 36, pp. 1018-1019, 2000.

Z.S. Guo, "Strain and Temperature Monitoring of Asymmetric Composite Laminate using FBG Hybrid Sensors", Structural Health Monitoring, Vol 6, pp. 191-197, 2007.

W. Hei, H. Cheng, J. Mei and D. Jiang, "Direct measurement of strain-optic effect in fiber Bragg gratings", Proceedings of the 15 $5^{\text {th }}$ Optical Fiber Sensors Conference, Vol. 1, pp. 171-174, 2002.

K. O. Hill, Y. Fujii, D. C. Johnson, and B. S. Kawasaki, "Photosensitivity in optical fiber waveguides: Application to reflection filter fabrication," Applied Physics Letters, vol. 32, pp. 647-649, 1978.

K. 0. Hill, Malo, F. Bilodeau, D. C. Johnson, and J. Albert, "Bragg gratings fabricated in monomode photosensitive optical fiber by UV exposure through a phase mask", Applied Physics Letters, Vol. 62, 1993.

D. R. Hjelme, L. Bjerkan, S. Neegard, J. S. Rambech, and J. V. Aarsnes, "Application of Bragg grating sensors in the characterization of scaled marine vehicle models," Applied Optics, Vol. 36, pp. 328-336, 1997.

J. Holt, Structural Alloys Handbook, CINDAS/Purdue University, West Lafayette, IN, 1996.

K. Hotate, "Distributed dynamic strain measurement using a correlation-based Brillouin sensing system”, IEEE Photonics Technology Letters, Vol. 15, pp. 272-274, 2003.

A. Hongo, S. Kojima and S. Komatsuzaki, "Applications of fiber Bragg grating sensors and high-speed interrogation techniques", Structural control and health monitoring, Vol. 12, pp. 269-282, 2005. 
A. Ivanov and J. Albert, "Bent tilted fiber Bragg gratings for temperature-independent vibration sensing in vehicles", Proceedings of Photonics in the Trasportation Industry: Auto to Aerospace, SPIE, 6758-2, 2007 .

A. Ivanov and J. Albert, "Tilted fiber Bragg gratings stubs for vibration and bend sensing", Proceedings of the OSA Topical Meetings on Bragg Gratings, Photosensitivity and Poling in Glass Waveguides, paper JWA49, 2007b.

S.W. James, R.P. Tatam, A. Twin, M. Morgan and P. Noonan, "Strain response of fibre Bragg grating sensors at cryogenic temperatures" Measurement Science and Technology, Vol. 13, pp. 1535-1539, 2002 .

J.M. Jewell, "Thermooptic Coefficients of Some Standard Reference Material Glasses", Journal of American Ceramic Society, Vol. 74(7), pp. 1689-1692, 1991

H.K Kang, D.H. Kang, H.J. Bang, C.S. Hong and C.G. Kim, "Cure monitoring of composite laminates using fiber optic sensors" Smart Materials and Structures, Vol. 11(2), pp. 279-287, 2002.

K. S. Lee, and T. Erdogan, "Fiber mode coupling in transmissive and reflective tilted fiber gratings," Applied Optics, Vol. 39, pp. 1394-1404, 2000.

P. J. Lemaire, R. M. Atkins, V. Mizrahi, and W. A. Reed, "High pressure H2 loading as a technique for achieving ultrahigh UV photosensitivity and thermal sensitivity in $\mathrm{GeO} 2$ doped optical fibres,"

Electronics Letters, Vol. 29, pp. 1191-1193, 1993.

D.B. Leviton and B.J. Frey, "Temperature-dependent absolute refractive index measurements of synthetic fused silica", Proceedings of, Optomechanical Technologies for Astronomy, SPIE, Vol. 6273, 62732K, 2006.

D. Li, H. Li, L. Ren and G. Song, "Strain transferring analysis of fiber Bragg grating sensors", Optical Engineering, Vol. 45(2), 024402, 2006.

J.M. Lifshitz and A. Rotem "Determination of Reinforcement Unbonding of Composites by a Vibration Technique" Journal of Composite Materials, Vol. 3, pp. 412-423, 1969.

Y. Liu, L. Zhang, J.A.R. Williams, and I. Bennion, "Optical Bend Sensor Based on Measurement of Resonance Mode Splitting of Long-Period Fiber Grating", Photonics Technology Letters, Vol. 12(5), pp. 531-533, 2000

T.L. Lowder, R.S. Gibson, K.H. Smith, R.H. Selfridge, and S.M. Schultz, "Unique characteristics of surface relief fiber Bragg gratings and their application to multi axis sensing", Proceedings of Smart Structures and Materials 2006, SPIE, Vol. 6167, 616709, 2006.

S. Magne, S. Rougeault, M. Vilela and P. Ferdinand, "State-of-strain evaluation with fiber Bragg grating rosettes: application to discrimination between strain and temperature effects in fiber sensors" Applied Optics, Vol. 36(36), pp.9437-9447, 1997.

R. Maier, W. MacPherson, J. Barton, J. Jones, S. McCulloch, G. Burnell, "Temperature dependence of the stress response of fiber Bragg gratings", Measurement Science and Technology, Vol. 15, pp. 1601$1606,2004$. 
I.H. Malitson, "Interspecimen comparison of the refractive index of fused silica," Journal of Optical Society of America, Vol. 55(10), pp.1205-1209, 1965.

G. Meltz, W. W. Morey, and W. H. Glenn, "Formation of Bragg gratings in optical fibers by a transverse holographic method", Optics Letters, Vol. 14(15), pp. 823-825, 1989

MicronOptics Inc. "Temperature compensated strain sensor", web resource http://www.micronoptics.com/os410.htm, January 2008,

N. Mrad, A. Ivanov, J. Albert and G. Xao, "Characterization of fibre Bragg gratings for temperature and strain sensing", proceedings of Structural Health Monitoring and Nondestructive Evaluation SPIE, Vol. 6529, pp. 65290T,2007.

W.W.Morey, G.Meltz and W.H.Glenn, "Fiber optic Bragg grating sensors", Proceedings of Fiber Optic and Laser Sensors VII, SPIE, Vol. 1169, pp. 98-107, 1989.

A. Othonos, and K. Kalli, Fiber Bragg Gratings: Fundamentals and Applications in Telecommunications and Sensing, Boston: Artech House, 1999.

Y.J. Rao,Y.P. Wang,Z.L. Ran, and T. Zhu, "Novel Fiber-Optic Sensors Based on Long-Period Fiber Gratings Written by High-Frequency CO2 Laser Pulses", Journal of lightwave technology, Vol. 21(5), pp. 1320-1327, 2003.

F. Ravet, L. Zou, X. Bao, T. Ozbakkaloglu, M. Saatcioglu, and J. Zhou, "Distributed Brillouin sensor for structural health monitoring", Canadian Journal of Civil Engineering, Vol. 34, pp. 291-297, 2007.

R.T. Schermer, "Mode scalability in bent optical fibers", Optics Express, Vol. 15(24), pp. 15674-15701, 2007.

X. Shu, Y.Liu, D.Zhao, B.Gwandu, F.Floreani, L.Zhang, and I.Bennion, "Dependence of temperature and strain coefficients on fiber grating type and its application to simultaneous temperature and strain measurement", Optics Letters, Vol. 27(9), pp. 701-703, 2002.

P. Sivanesan, J.S. Sirkis, Y. Murata, S. G. Buckley, "Optimal wavelength pair selection and accuracy analysis of dual fiber grating sensors for simultaneously measuring strain and temperature," Optical Engineering, Vol. 41(10), pp. 2456-2463, 2002.

N.G. Skinner and J.L. Maida, "Downhole Fiber-optic Sensing: The Oilfield Service Provider's Perspective" Proceedings of Fiber Optic Sensor Technology and Applications III, SPIE, Vol. 5589, pp. 206-220, 2004.

K. Sugden, L. Zhang, J.A.R. Williams, R.W. Fallon, L.A. Everall, K. E. Chisholm, and I. Bennion, "Fabrication and characterization of bandpass filters based on concatenated chirped fiber gratings," Journal of Lightwave Technology, Vol. 15, pp. 1424-1432, 1997.

Z.Wang, M.Han, F.Shen and A.Wang, "Ultra-short fiber Bragg grating intrinsic Fabry-Perot interferometric sensors for quasi-distributed strain and temperature sensing", Proceedings of Sensors and Smart Structures Technologies for Civil, Mechanical, and Aerospace Systems, SPIE, Vol. 6529. pp.6529-35, 2007. 
Z.-J. Wu, Z.-S. Guo, L. Wan, B.M. Zhang, and S.Y. Du. "Measurement of process-induced stresses in composite laminates by FBG sensors" Proceedings of SAMPLE pp. 3217-3225, 2004.

M. G. Xu, L. Reekie, Y. T. Chow and J. P. Dakin, "Optical in-fiber grating high pressure sensor", Electronics Letters, Vol. 29(4), pp. 398-399, 1993.

M.G.Xu, J.L.Archambault, L.Reekie and J.P. Datkin, "Discrimination between strain and temperature effects using dual-wavelength fiber grating sensors", Electronics Letters, Vol. 30(13), pp. 1085-1087, 1994.

G.W. Yoffe, P.A. Krug, F. Ouellette, and D.A. Thorncraft, "Passive temperature-compensating package for optical fiber gratings", Applied Optics, Vol. 34(30), pp. 6859-6861, 1995.

C. Yong, D.G. Zimcik, V.K. Wickramasinghe, and F. Nitzsche, "Development of the Smart Spring for Active Vibration Control of Helicopter Blades" Journal of Intelligent Material Systems and Srtuctures, Vol. 15, pp.37-48, 2004.

X. Zhang, Z. Wu and B. Zhang, "Strain dependence of fiber Bragg grating sensors at low temperature", Optical Engineering, Vol. 45(5), 054401, 2006.

D. Zhao, K. Zhou, X. Chen, L. Zhang, I. Bennion, G. Flockhart, W.N. MacPherson, J. S. Barton and J.D.C. Jones, "Implementation of vectorial bend sensors using long-period gratings UV-inscribed in special shape fibres", Measurement science and technology, Vol. 15, pp. 1647-1650, 2004. 